REMOTELY SENSING THE SPECIES OF INDIVIDUAL TREES 



\title{
REMOTELY SENSING THE SPECIES OF INDIVIDUAL TREES
}

\author{
DISSERTATION \\ to obtain \\ the degree of doctor at the University Twente, \\ on the authority of the rector magnificus, \\ Prof.dr. T.T.M. Palstra, \\ on account of the decision of the graduation committee \\ to be publicly defended \\ on Friday 31 January 2020 at $16.45 \mathrm{hrs}$
}

by

Yifang Shi

Born on 21 July 1990

in Henan, China 
This thesis is approved by:

Prof. dr. A. K. Skidmore, supervisor

A/Prof. dr. T. Wang, co-supervisor

ITC dissertation number 376

ITC, P.O. Box 217, 7500 AE Enschede, The Netherlands

ISBN: 978-90-365-4953-0

DOI: $10.3990 / 1.978903654953-0$

Cover designed by Marc Abbink

Printed by ITC Printing Department

Copyright (C) 2020 by Yifang Shi

\section{UNIVERSITY OF TWENTE.}

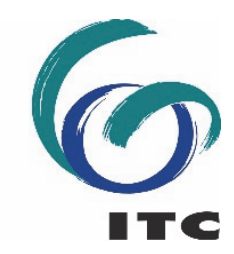


Graduation committee:

\section{Chair}

Prof. dr. ir. A. Veldkamp

University of Twente

\section{Supervisor}

Prof. dr. A. K. Skidmore

University of Twente

\section{Co-supervisor}

A/Prof. dr. T. Wang

University of Twente

\section{Members}

Prof. dr. W. Verhoef

University of Twente

Prof. dr. V. G. Jetten

University of Twente

Prof. dr. F. V. Coillie

Ghent University

Prof. dr. C. Atzberger

University of Natural Resources and Life

Sciences 
To my family 


\section{Acknowledgements}

This $\mathrm{PhD}$ journey has been an amazing, precious and unforgettable period in my life. My sincerest appreciation to all who have contributed to this thesis and supported me throughout my $\mathrm{PhD}$ journey.

First and foremost, I would like to express my sincere thanks to my promotor, Prof. Andrew K. Skidmore. Andrew, thank you for your valuable guidance, insightful advice, and continuous support. The discussion we had taught me how to view scientific questions from different perspectives and gradually I became more and more confident when you challenge me. Thanks to your encouragement and trust, I dare to think bigger and explore the topic more freely. I am very grateful for your patient guidance and critical comments during my $\mathrm{PhD}$ research. You always remind me the importance of "critical thinking" and "think out of the box", which I will benefit from not only for my $\mathrm{PhD}$ research, but also for the rest of my life. Thank you for recommending me to spend a period of my $\mathrm{PhD}$ journey in DLR, Germany, which greatly enriched my $\mathrm{PhD}$ and life experience.

I am deeply grateful to my daily supervisor, Dr. Tiejun Wang, who spotted my potential to do a $\mathrm{PhD}$ in my application four years ago and gave me the chance to experience a new life journey in the Netherlands. Thank you for encouraging me to challenge a completely new topic for my $\mathrm{PhD}$ - tree species mapping using LiDAR and hyperspectral data. It was not always easy at the beginning, but later I started to enjoy and love the topic and questions occurred in my research. I appreciate your guidance and support in writing the proposal and later the papers. Your prompt feedback and insight comments always helped me shape my idea into a better story. I also learned from you how to manage the research progress efficiently and how to explore the potential of myself for future career. Thank you for always being there for me and helping me overcome obstacles through my $\mathrm{PhD}$ journey.

I would like to express my truly gratitude to the support from German Aerospace Center (DLR), where I spent six months as a scientific visitor. Special thanks go to Dr. Uta Heiden and Dr. Stefanie Holzwarth for helping me settle down in Munich, preprocessing hyperspectral data as well as giving me valuable advice for my research. Thanks Anna Wendleder who gave me warm company during my stay and always kept our house tidy and clean. Thanks Ya-Lun Tsai and Zhongyang $\mathrm{Hu}$, I had a memorable time in Gilching. 
I sincerely acknowledge the support of the Data Pool Initiative in Bavarian Forest National Park. Thanks to Dr. Marco Heurich, for his support in suggesting potential study sites and assisting fieldwork. Your knowledge of ecology and LiDAR always gave me special insight for tree species mapping. Thanks Joe Premier for his help on data collection and logistical facilities.

I have received a lot of supports from my colleagues in ITC during my $\mathrm{PhD}$. Without them, this PhD journey would not have been possible. Thanks Jing, Haidi and Tawanda, who conducted the fieldwork together with me. We have visited many plots together with all kinds of unexpected conditions and thanks for your company, I enjoyed very much the time we spent in the forests. Thanks to Sam who helped me with English editing for my papers. I am grateful to Esther Hondebrink for being always delightful, kind and helpful. Thanks to Loes Colenbrander for her friendly assistance during my study in ITC. My sincere gratitude goes to Benno and Job for their help in poster design and equipment assistance, to Willem for his technical assistance, and to Caroline for lab equipment assistance. I would like to thank Prof. Andy Nelson for his support at NRS department. Thanks go to all the colleagues in NRS, they are Roshanak, Thomas, Eddy, Yousif, Henk, Louise, Anton, Michael, Eddy, Panagiotis, Wieteke, Elnaz, Sugandh, Trini, Festus, Xi, Nina, Anna, Marcelle, Haili, Xin, Abebe, Alby and etc. Thanks to Marga and Carla from ITC library for their kind help and assistance for the $\mathrm{PhD}$ tutorial.

I extend my special gratitude to my officemates and friends in Room 4-110, Dr. Xi Zhu, Dr. Elnaz Neinavaz, Dr. Festus Wanderi Ihwagi, Dr. Maria Fernanda Buitrago, and Xin Zong. We have had so many memorable moments in and out of the office. In particular, I would like to thank Hong and Xi for their company, comfort and support from the first day of my $\mathrm{PhD}$. Your friendship and support have made my $\mathrm{PhD}$ journey so much more rewarding. Special thanks to Marc, who always surprises me in every possible way and shares the most memorable moments with me. I also thank all my fellow PhD students and colleagues in ITC: Fangyuan, Yiwen, Zhihui, Linlin, Xu, Xiaolong, Ruodan, Xiaoling, Peiqi, Yijian, Junping, Lilin, Wen, Mengna, Yuhang, Zhenchao, Ruosha, Chengliang, Shaoning, Pei, Tina, Parinaz, Mitra, Tonny, Dimitris, Bryan, Sam and so on.

Finally, my deepest gratitude goes to my parents, without whom I would not have reached this millstone. To my dearest mother and father, thank you for your unconditional love and continuous support. Thank you for respecting and understanding my choice and always being there for me in my life. 


\section{Table of Contents}

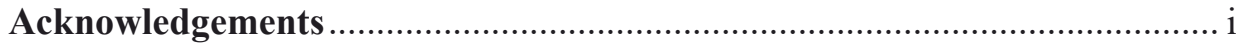

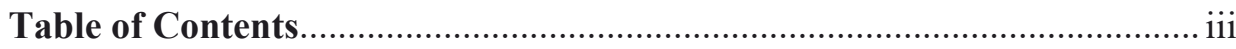

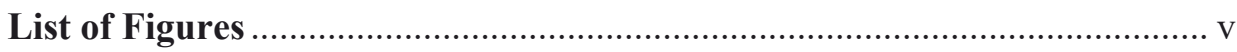

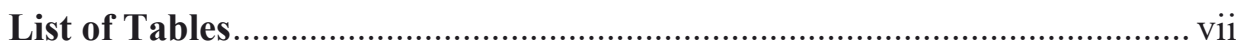

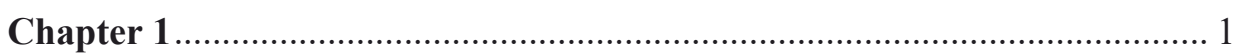

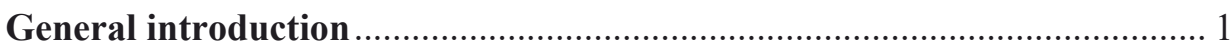

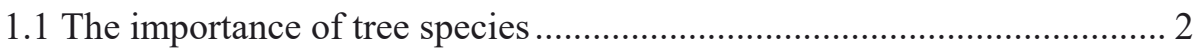

1.2 From field-based to remote sensing-based tree species mapping.............. 2

1.3 The species of individual trees mapped by remote sensing sensors.......... 3

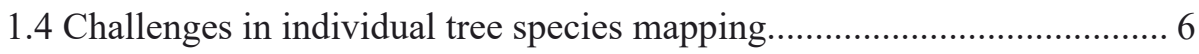

1.5 Objectives and research questions........................................................... 7

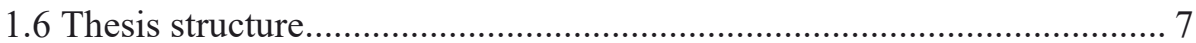

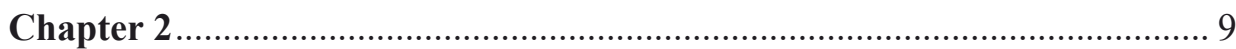

Important LiDAR metrics for discriminating tree species ......................... 9

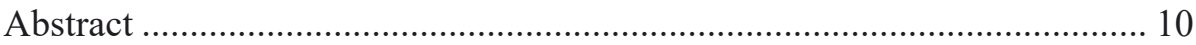

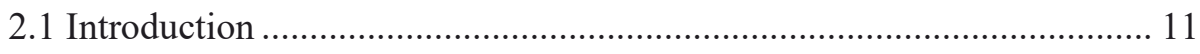

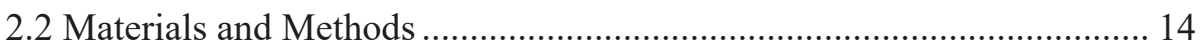

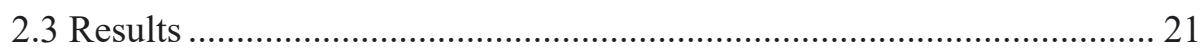

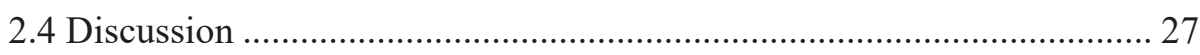

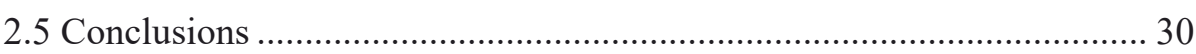

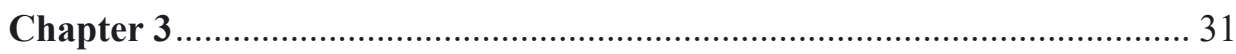

Improving LiDAR-based tree species mapping using multi-temporal CIR

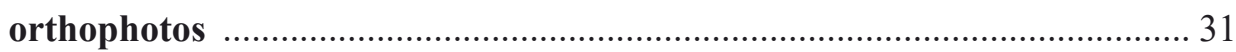

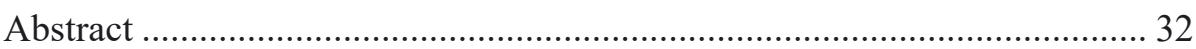

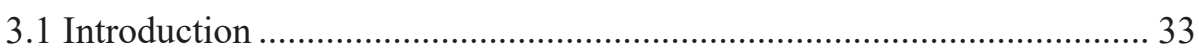

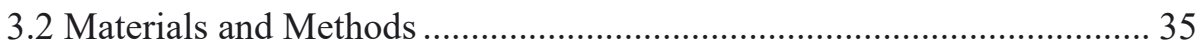

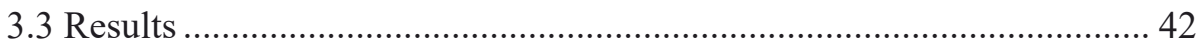

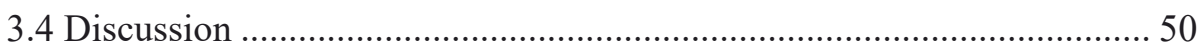

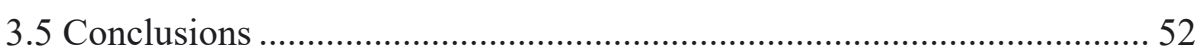

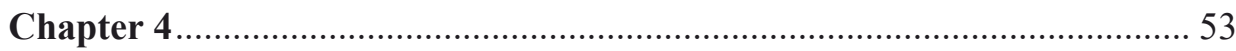

Tree species classification using remotely sensed plant functional traits .. 53

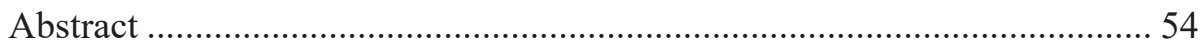

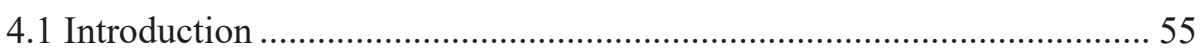

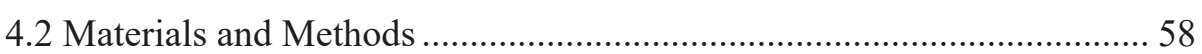

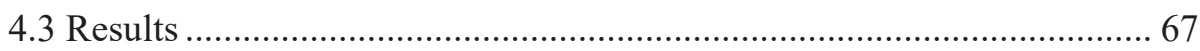

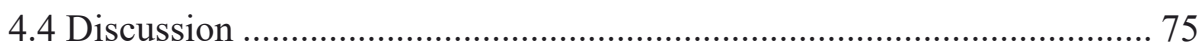




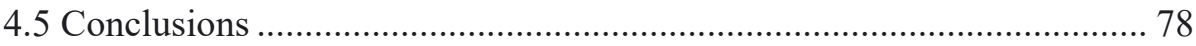

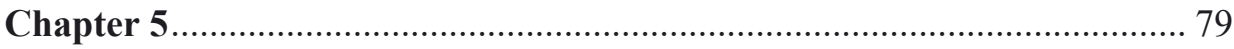

Mapping individual silver fir trees in a Norway spruce dominated forest 79

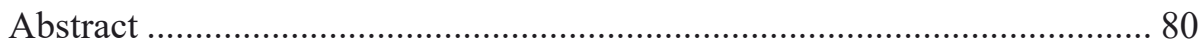

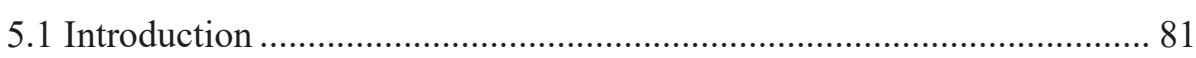

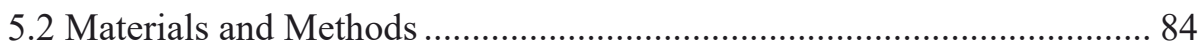

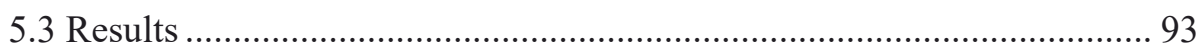

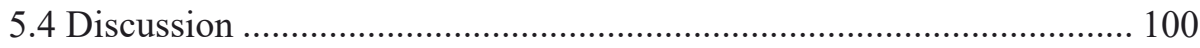

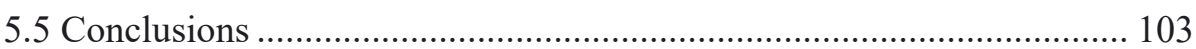

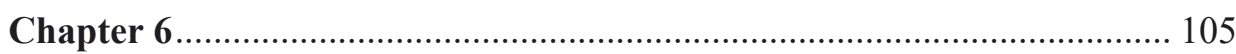

Synthesis: Mapping individual tree species using multi-source remotely

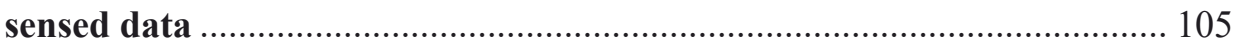

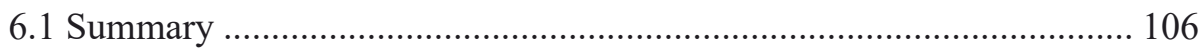

6.2 The potential of geometric and radiometric features derived from LiDAR

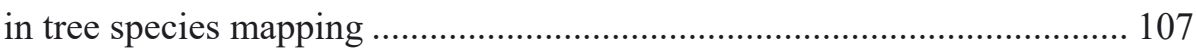

6.3 The contribution of multi-temporal airborne remotely sensed data in tree

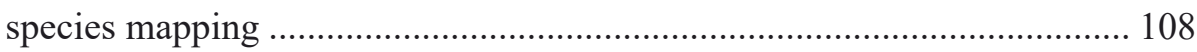

6.4 The role of remotely sensed plant functional traits in tree species mapping

6.5 Improving mapping accuracy of single tree species by connecting remotely sensed features and species-specific traits................................... 112

6.6 The role of machine learning techniques in tree species mapping ........ 113

6.7 Applications of remote sensing in forestry improve the efficiency of

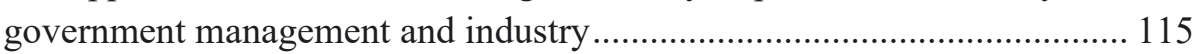

6.8 Future work and broader implications.................................................. 116

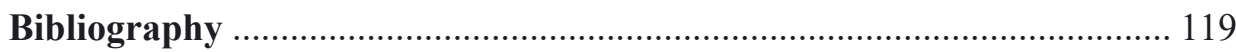

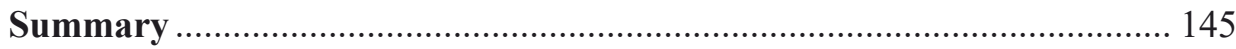

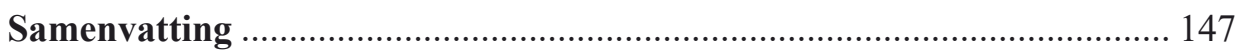

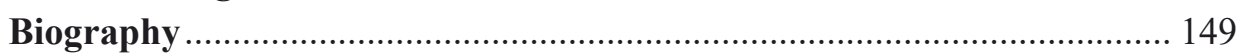




\section{List of Figures}

Fig. 3.1 Location of the two study sites in Bavaria Forest National Park, Germany 36

Fig. 3.2 The digital CIR orthophotos of a sample area from 2015 (a), 2016 (b) and 2017 (c). The 3D segmentation of tree crown is displayed with the background of the LiDAR-derived canopy height model (d) 39

Fig. 3.3 The cross-correlation matrix of the texture features derived from multitemporal digital CIR orthophotos (a) and the combined correlogram with the significance test of 2015 (b). Blue colours indicate positive correlations and red colours indicate negative correlations. The insignificant values $(p>0.05)$ were marked with black crosses. The features were sorted by hierarchical clustering order (black rectangles) 43

Fig. 3.4 The relative importance of the selected variables for tree species classification. 48

Fig. 3.5 Interspecies comparison of different variables derived from multitemporal digital CIR orthophotos and LiDAR data. The first row is the average gray level of the NIR band (MEAN_NIR) from 2015, 2016 and 2017 digital CIR orthophotos; the second row is the dissimilarity of the NIR band (DIS_NIR) from 2015, 2016 and 2017 digital CIR orthophotos; and the last row is the mean intensity of first-or-only returns (Imean_first), the mean value of echo width (Ewmean), and the tree height (Height) derived from airborne LiDAR data.... 49 Fig. 4.1 Airborne LiDAR and HySpex data collections and the location of the two study sites in the Bavarian Forest National Park. 59

Fig. 4.2 3D individual tree segmentation using the approach of Yao et al. (2013) on (a) CHM with tree crown and tree top derived from LiDAR data, and (b) HySpex data with tree top derived from LiDAR data. 63

Fig. 4.3 Scatter plots of measured and estimated $\mathrm{Cw}$ (a), $\mathrm{Cm}$ (b) and $\mathrm{Cab}$ (c) using the INFORM model. Data points are derived from the measured (validation) dataset collected from the study site, and each point represents a sample tree (215 sample trees in total).....

Fig. 4.4 The mean reflectance value $(\times 1000, \pm 1$ standard deviation $)$ of five tree species at $400-2498 \mathrm{~nm}$ wavelengths. 69

Fig. 4.5 The relative importance and ranking of the selected variables for tree species classification under different combinations (a) LiDAR+ HSI+ PFTs, (b) LiDAR+ HSI and (c) LiDAR+ PFTs. 
Fig. 4.6 Box plots of equivalent water thickness (a), dry matter content (b), mean intensity of first-or-single returns (c), mean value of echo width (d), first derivation of band $1771.1 \mathrm{~nm}$ (e), SWIR_VI (f) among five tree species 74 Fig. 4.7 Map of individual tree species classification for an example area (located in site A) in Bavarian Forest National Park 75

Fig. 5.1 Airborne LiDAR and HySpex flight area and the location of two study sites in the Bavarian Forest National Park, Germany... 85

Fig. 5.2 Segmented individual tree crowns for the two study site. 87

Fig. 5.3 The mean reflectance value $(\times 1000, \pm 1$ standard deviation) of five species (i.e. beech, birch, fir, maple and spruce) at 400-2498 nm wavelengths derived from HySpex data acquired in 2015 and 2016, respectively. 94 Fig. 5.4 Box plots of four vegetation indices (i.e. ACI2, DWSI2, RVSI and SWIR_VI) derived from 2015 (first row) and 2016 (second row) among sample tree species. 95

Fig. 5.5 Box plots of the percentage of first returns above $2 \mathrm{~m}$ (a), the percentage of all returns above $2 \mathrm{~m}$ (b), 99th percentile of tree height (c), the intensity of 95th percentile of normalized tree height (d), the mean echo width of single returns (e), the mean height of first returns (f) among sample tree species. 96 Fig. 5.6 The spectral separability index (SI) between fir and other four tree species (i.e. maple, beech, birch, and spruce) in the year of 2015 and 2016. 0 indicates the lowest SI and 1 indicates the highest SI between two species. 97 Fig. 5.7 The normalized importance of selected features from the combination of each year HySpex and LiDAR data for fir classification. 100

Fig. 5.8 Maps of fir trees in two study sites (500 $\mathrm{m} \times 500 \mathrm{~m}$ for each site) in the Bavarian Forest National Park. The crown of fir trees are highlighted in yellow. The point clouds of mapped fir are highlighted in red. 100

Fig. 5.9 The crown shape of a silver fir tree (a) and a Norway spruce tree (b). (Photos by Rainer Simonis) 102

Fig. 6.1 An example of the LiDAR returns under leaf-on and leaf-off conditions 109

Fig. 6.2 Plant functional traits measured in the field among six tree species in the Bavarian Forest National Park, Germany. 111

Fig. 6.3 Sample workflow for comparing machine learning methods for tree species classification (Marrs and Ni-Meister 2019). 


\section{List of Tables}

Table 2.1 Characteristics of the two pilot study sites

Table 2.2 The sample size, mean height and standard deviation (SD) of each tree species in site A and site $\mathrm{B}$

Table 2.3 Description of the 37 generated LiDAR metrics ............................... 19

Table 2.4 Selected LiDAR metrics derived under leaf-on and leaf-off conditions and their Mean Decrease Accuracy (MDA). The top 7 metrics indicated by the asterisk (*) from each condition (i.e. leaf-on and leaf-off) were used as final input metrics for tree species classification.

Table 2.5 Comparison of overall accuracy and kappa coefficient for tree species classification using leaf-on, leaf-off and combination of leaf-on and leaf-off LiDAR metrics.

Table 3.1 Detailed information of multi-temporal digital CIR orthophotos used in this study. 37

Table 3.2 Description of generated texture features. In formulas, $i$ and $j$ are row and column numbers, respectively. $N$ is the total number of pixels. $u i, u j, \sigma i 2$, and $\sigma j 2$ are the means and standard deviations of $P i$ and $P j . P i, j$ is the normalized co-occurrence matrix. 41

Table 3.3 Selected variables derived from LiDAR and digital CIR orthophotos for classification 44

Table 3.4 Confusion matrices for five tree species using different selections of variables. PA is producer's accuracy, UA is user's accuracy, OA is overall accuracy..... 46

Table 3.5 McNemar's test for pairwise comparison between classification results using different combinations. CIR $(\times 3)$ means all three years of digital CIR orthophotos; CIR $(\times 2)$ means any two years of digital CIR orthophotos; CIR $(\times 1)$ means any one year of digital CIR orthophoto. ${ }^{* *}: p<0.001 ; * *: p<0.01 ; *: p$ $<0.05$; NS: $p>0.05$.

Table 3.6 Top five most important variables for discriminating each tree species in the classification. See Table 3.3 for the definitions of the metrics. 49 Table 4.1 Summary of the sample trees and three plant functional traits measured in site $A$ and site B: equivalent water thickness $(\mathrm{Cw})$, leaf mass per area $(\mathrm{Cm})$, leaf chlorophyll (Cab). 61

Table 4.2 Description of generated hyperspectral features ................................ 64 Table 4.3 Input parameters and ranges used for generating the LUT from the INFORM model 
Table 4.4 Selected variables derived from LiDAR and hyperspectral data for

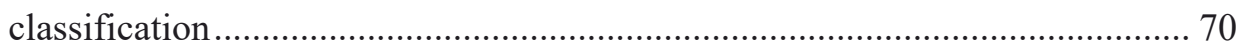

Table 4.5 Confusion matrix of classification performance ………….............. 71

Table 4.6 The classification results using different sets of variables (LiDAR:

LiDAR derived metrics, HSI: spectral features, PFTs: retrieved plant functional traits) 71

Table 4.7 McNemar's test for pairwise comparison between classification results generated from different combinations. ${ }^{* * *}: p<0.001$; $^{* *}: p<0.01 ; *: p<0.05$;

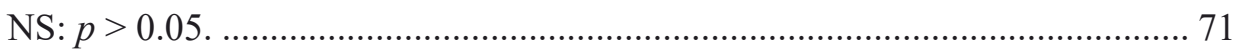

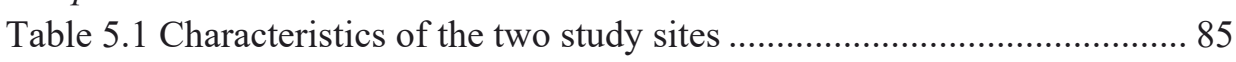

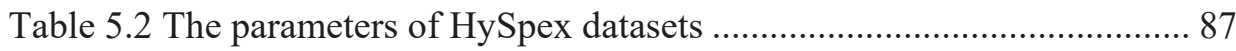

Table 5.3 List of generated LiDAR metrics and hyperspectral features ........... 89

Table 5.4 Description of one-class classifiers ................................................... 92

Table 5.5 Selected features derived from hyperspectral and LiDAR data ........ 98

Table 5.6 One-class classification results of fir trees from hyperspectral and LiDAR data using three different classifiers 99

Table 5.7 McNemar's test for pairwise comparison between classification results using different classifiers. NS: $p>0.05 .{ }^{* * *} p<0.001 . * * p 0.01 .{ }^{*} p<0.05 . .99$ 
Chapter 1

General introduction 


\subsection{The importance of tree species}

Understanding and quantifying the nature of tree species is important from both ecological and economic perspectives. Information on composition, distribution, and diversity of tree species is of primary significance in the planning and implementation of biodiversity conservation efforts (Suratman 2012). The accurate mapping of individual trees at species level can provide a fundamental basis for sustainable forest management, ecosystem services assessment, as well as biodiversity monitoring (Dalponte et al. 2012; Shang and Chazette 2014).

Numerous studies in recent years highlighted the importance of tree species maps either as a standalone product for forest management (e.g. Dalponte et al. 2012; Heinzel and Koch 2012; Richter et al. 2016) or as an essential input for speciesspecific growth and yield models (e.g. Ghosh et al. 2014; Vauhkonen et al. 2014) or invasive tree species monitoring (e.g. Piiroinen et al. 2018; Somers and Asner 2013b). Due to the importance of tree species information, it is crucial to build a reliable tree species mapping system for those applications, such as resource management, biodiversity assessment, ecosystem services assessment and nature conservation (Wagner et al. 2018).

\subsection{From field-based to remote sensing-based tree species mapping}

Conventionally, identification and mapping of tree species are carried out by field inventory. However, inventories conducted in the field by trained professionals are expensive, time-consuming and not applicable to large or isolated areas (Kim 2007). During the last decades, both field-based inventories and remote sensing approaches have been used for tree species mapping (Ghosh et al. 2014). While field-based measurements have been criticized for requiring more time, manpower and economic resources (Mairs 1976), information derived from remotely sensed data has been promoted as providing an alternative (Holmgren and Thuresson 1998). Remote sensing approaches allow not only lower measurement costs, but also access to spatially-continuous data collection over large portions of the Earth's surface (Asner and Martin 2009; Palmer et al. 2002), including remote forests or areas where conditions are dangerous. As one of the most popular forms of remote sensing of forests in the early $90 \mathrm{~s}$, the ability of aerial photographs to provide tree species information is well valued and has been used for decades in forest inventory (Loetsch and Haller 1964). However, manually interpretation of aerial photographs by human operators remains time- 
consuming and subjective. In addition, visual interpretation may not always fully reveal information about the characteristics of individual trees, while the variability among same tree species and the similarity between different tree species could significantly increase the challenges.

More recently emerged remote sensing sensors (e.g. multispectral, hyperspectral and Light Detection and Ranging (LiDAR) systems) represent an efficient and potentially economical way of depicting the characteristics of tree species by capturing the spectral and structural signatures, providing valuable information for forest inventory and tree species mapping on larger geographic scales (Sothe et al. 2019). While field-based measurements provide accurate information at local scales allowing validation of remotely sensed data, it remains insufficient to regularly sample large or poorly-accessible areas, approaches combining field and remotely sensed data could potentially provide cost-effective means to map tree species at different scales (Ganivet and Bloomberg 2019).

\subsection{The species of individual trees mapped by remote sensing sensors}

In order to capture the complex inter-species and intra-species spectral variability and structural variations of individual trees resulting from genetic patrimony and difference in environmental and physical factors (e.g. geology and edaphic conditions and natural phenological changes), passive remote sensing sensors (i.e. airborne multispectral or hyperspectral sensor) need to equip with numerous, contiguous spectral bands along with a high spatial resolution in relation to the scale of tree crowns, while active remote sensing sensors (i.e. airborne LiDAR) should be able to capture the detailed geometric characteristics of the individual tree that different from other species (Naidoo et al. 2012).

\subsubsection{Passive remote sensing - multispectral and hyperspectral systems}

Passive optical sensors can be divided into multispectral and hyperspectral (also called imaging spectroscopy) systems. Whereas most of the multispectral sensor systems typically have $4-8$ bands, hyperspectral imagery is acquired in narrow, contiguous bands that can cover the visible (VIS), near-infrared (NIR) and shortwave-infrared (SWIR) portions of the electromagnetic spectrum (400-2500 $\mathrm{nm})$. Both multispectral and hyperspectral systems provide useful information to separate tree species by measuring the spectral response of directional electromagnetic radiation emitted by the sun and reflected by the canopy (and 
other surfaces) in sensor-specific wavelengths regions (Fassnacht et al. 2016). At canopy level, the amount of radiation that is reflected in the different wavelengths regions is related to (1) plant chemical properties of the tissue which include water, photosynthetic pigments and structural carbohydrates (Ali et al. 2017; Asner 1998), (2) leaf morphology (thickness of cell-walls, air spaces and cuticle wax) (Clark et al. 2005), as well as (3) canopy structure (leaf and branch density, angular distribution, clumping) and tree size compared to neighboring trees (Leckie et al. 2005) which also depend on view-illumination geometry (Korpela et al. 2011). These properties vary not only with species but also with vertical leaf area density, leaf age and health status (Fassnacht et al. 2016).

Another useful source of information that may be captured from passive optical remote sensing for tree species discrimination is plant phenology. Plant phenology embraces very obvious processes such as the coloring of leaves in deciduous temperate forests in autumn due to leaf senescence, and the intense green colors of fresh leaves and needles in spring time as well as flowering events (Fassnacht et al. 2016). Since plant phenology varies with species, speciesspecific knowledge of phenology is preferable over broad knowledge of forest phenology (Chuine and Beaubien 2001). It is therefore desirable to align the time of image acquisition with the phenological cycle of the species under investigation (Gärtner et al. 2016). Multi-temporal optical data acquisitions provide a way to incorporate the spectral variation of species phenology for tree species classification.

\subsubsection{Active remote sensing - LiDAR system}

Recent developments in active remote sensing, particularly the light detection and ranging (LiDAR) technique, has shown great potential for individual tree species mapping due to its capability of capturing three-dimensional (3D) information of objects of interest. Airborne LiDAR is a favored data source for individual tree delineation, while also providing valuable geometric and radiometric information for tree species discrimination. While the geometric metrics describe the geometric structure of trees (e.g. crown shape, tree height and crown volume), the radiometric metrics refer to specific echo parameters that are extracted from the received waveform (e.g. the backscatter cross-section, the energy of laser points, and the distance between two waveform echoes) (Koenig and Höfle 2016; Wagner 2010). These properties can all vary within and between tree species and are at least partly complementary to the data gathered by passive optical remote sensing sensors (Alonzo et al. 2014). 
Particularly, intensity of the backscattered laser signal is additionally related to foliage type, leaf size, leaf orientation, leaf clumping and foliage density (Kim et al. 2009; Korpela et al. 2010; Suratno et al. 2009). LiDAR intensity-related features were found to be amongst the most relevant predictors in numerous studies (e.g. Hovi et al. 2016; Korpela et al. 2010; Ørka et al. 2009; Vauhkonen et al. 2010a). Furthermore, multi-temporal LiDAR acquisitions (leaf-on and leafoff) have also been used for improving tree species discrimination since they may capture the foliage change between leaf-on and leaf-off conditions, such as the missing foliage and a thereby notably higher number of LiDAR returns on the ground and the stems which decreases the average height of the canopy surface model (Kim et al. 2009; Wasser et al. 2013).

\subsubsection{Thermal and Synthetic Aperture Radar (SAR) systems}

Compared to the abovementioned sensor types (i.e. multispectral, hyperspectral and LiDAR systems), there are fewer studies focused on thermal and Synthetic Aperture Radar (SAR) systems for tree species mapping. In the mid-infrared and thermal infrared part of the spectrum contrasting observations have been made. Salisbury (1986) presented leaf level thermal infrared spectra of beech (Fagus grandifolia), red oak (Quercus rubra) and two cherry species (Prunus sp.) and identified well-defined spectral features that differed notably across the four species. Ribeiro da Luz and Crowley (2007) found that the thermal infrared signal associates with several plant chemical and structural compounds such as cellulose, silica, and oleanolic acid, and they also pointed out that the signal in the thermal infrared domain is much more species-specific than the reflectance signal observed in the VIS-SWIR region (Fassnacht et al. 2016). Meanwhile, most SAR studies focused on the discrimination of broad forest types in the framework of land-cover classification omitting the species level. Forest information by SAR relates mainly to canopy structure and water content (Fassnacht et al. 2016). The application of advanced polarimetric measures to separate tree species has been investigated in a number of studies (e.g. Knowlton and Hoffer 1981; Maghsoudi et al. 2012; Wollersheim et al. 2011). However, environmental variables such as terrain condition has an influence on the Radar information, and scattering behaviour also varies with incident angle and wavelength which makes the whole system even more complex.

\subsubsection{Integration of various data sources for tree species mapping}

Combining complementary remote sensing data sources for tree species classification has been widely performed to improve mapping accuracy, 
especially with the synergistic use of airborne LiDAR and optical imageries (aerial photographs, multispectral and hyperspectral imageries) at the pixel or object level (Dechesne et al. 2017). Increasingly, LiDAR and either multispectral (e.g. Holmgren et al. 2008; Ørka et al. 2012) or hyperspectral (e.g. Alonzo et al. 2014; Dalponte et al. 2008; Liu et al. 2017) data are integrated at the pixel or object level for tree species classification and quantification of forest inventory parameters (e.g. Latifi et al. 2012; Sarrazin et al. 2012; Smits et al. 2012; Swatantran et al. 2011). Particularly, combining airborne LiDAR and hyperspectral datasets, as a state-of-the-art remote sensing technology, provides both horizontal and vertical information about tree species and has shown great potential in improving tree species discrimination (Zhang et al. 2016). For instance, at the pixel level, the integration of hyperspectral and LiDAR data increased both producer's (5.1-11.6\%) and user's (8.4-18.8\%) accuracies than using either dataset alone, as found by Jones et al. (2010). Dalponte et al. (2012) compared various combinations of LiDAR data (high and low density) with hyperspectral as well as multispectral data for tree species classification in a temperate forest. They found that the best classification accuracy was obtained when combining the LiDAR and hyperspectral datasets.

\subsection{Challenges in individual tree species mapping}

(1) From ecological and biological perspectives, tree species differ in their biochemical, biophysical and structural traits under different canopy conditions, resulting in diverge reflectance and architectures which can be captured by multispectral, hyperspectral and LiDAR data. However, high spectral and structural intra-species variability and inter-species similarity in natural mixed forests, due to phenological effects, differences in tree age and openness of canopies, shadowing effects, and environment variability, restrict tree species separability.

(2) From data collection and processing point of view, the "big data problem" followed by the emerging of new sensors became an acknowledged topic for researchers working on remote sensing. Data redundancy and high correlation between numerous features hamper the efficiency and accuracy of tree species classification. Valuable features that contribute to the discrimination of tree species need to be accurately identified.

(3) Many previous studies have focused on data-driven or algorithm-driven approaches and pursued an optimization of classification accuracy in specific study sites. Whether collected training samples are sufficient to adequately 
characterize investigated tree species may limit the understanding of the linkage between tree species and remote sensing signatures. An in-depth ecological and biological understanding of the relationship between species-specific traits and remote sensing observations for tree species classification has not been performed.

\subsection{Objectives and research questions}

The overall objective of this study is to accurately map individual tree species from remote sensing. The specific research questions are as follows:

(1) What kind of LiDAR metrics are important for mapping tree species? Do they perform differently under leaf-on and leaf-off conditions?

(2) How can multi-temporal digital aerial colour-infrared photographs further improve our understanding of tree species mapping?

(3) Can plant functional traits retrieved from hyperspectral data further improve the classification accuracy when used in conjunction with hyperspectral (spectral) features and LiDAR metrics?

(4) How to link species-specific traits with spectral and structural signatures derived from remotely sensed data to identify a focal tree species?

\subsection{Thesis structure}

This thesis comprises six chapters, including a general introduction, four core chapters, and a synthesis. Each core chapter has been provided as a standalone research article that has been published or submitted to peer-reviewed ISI journals.

The structure of the chapters is as follows: Chapter 1 presents the research background, identifies the existing challenges and proposes specific research questions in this thesis. Chapter 2 evaluates the important LiDAR metrics for discriminating tree species. Chapter 3 demonstrates how multi-temporal digital aerial colour-infrared photographs improve LiDAR-based tree species mapping. Chaper 4 performs tree species classification using plant functional traits retrived from LiDAR and hyperspectral data. Chapter 5 shows how species-specific traits linked with spectral and structral signatures derived from LiDAR and hyperspectral data for silver fir mapping. Chapter 6 concludes the thesis with a summary of significant findings in the thesis. The broader applications of this thesis in forest ecology research is outlined. 


\section{Chapter 2}

\section{Important LiDAR metrics for discriminating tree species *}

* This chapter is based on: Shi, Y., Wang, T., Skidmore, A.K., \& Heurich, M. (2018). Important LiDAR metrics for discriminating forest tree species in Central Europe. ISPRS Journal of Photogrammetry and Remote Sensing, 137, 163-174 


\begin{abstract}
Numerous airborne LiDAR-derived metrics have been proposed for classifying tree species. Yet an in-depth ecological and biological understanding of the significance of these metrics for tree species mapping remains largely unexplored. In this chapter, we evaluated the performance of 37 frequently used LiDAR metrics derived under leaf-on and leaf-off conditions, respectively, for discriminating six different tree species in a natural forest in Germany. We firstly assessed the correlation between these metrics. Then we applied a Random Forest algorithm to classify the tree species and evaluated the importance of the LiDAR metrics. Finally, we identified the most important LiDAR metrics and tested their robustness and transferability. Our results indicated that about $60 \%$ of LiDAR metrics were highly correlated to each other $(|r|>0.7)$. There was no statistically significant difference in tree species mapping accuracy between the use of leafon and leaf-off LiDAR metrics. However, combining leaf-on and leaf-off LiDAR metrics significantly increased the overall accuracy from 58.2\% (leaf-on) and $62.0 \%$ (leaf-off) to $66.5 \%$ as well as the kappa coefficient from 0.47 (leaf-on) and 0.51 (leaf-off) to 0.58 . Radiometric features, especially intensity related metrics, provided more consistent and significant contributions than geometric features for tree species discrimination. Specifically, the mean intensity of first-or-single returns as well as the mean value of echo width were identified as the most robust LiDAR metrics for tree species discrimination. These results indicate that metrics derived from airborne LiDAR data, especially radiometric metrics, can aid in discriminating tree species in a mixed temperate forest, and represent candidate metrics for tree species classification and monitoring in Central Europe.
\end{abstract}




\subsection{Introduction}

Discrimination of tree species is a major task undertaken in a wide range of environmental applications, such as biodiversity monitoring (Shang and Chazette 2014; Suratman 2012), ecosystem services assessment (Jones et al. 2010; Skidmore et al. 2015), invasive species detection and control (Boschetti et al. 2007), as well as sustainable forest management (Pcorona et al. 2006). Remote sensing can provide a valuable information source towards our understanding of ecosystem structure and function over large spatial scales (Baldeck et al. 2015). The identification and mapping of tree species is usually conducted through visual interpretation of aerial photographs by human experts coupled with forest inventory (in situ) plots, which is labour-intensive, time consuming and costly. More importantly, this method is not practical or applicable to large forested areas (Kim et al. 2009). Optical remote sensing such as airborne or spaceborne multispectral and hyperspectral images have been used to map tree species over the last few decades (e.g. Aspinall 2002; Boschetti et al. 2007; Feret and Asner 2013; Immitzer et al. 2012; Jones et al. 2010; Leckie et al. 2003; Leckie et al. 2005; Somers and Asner 2014). However, during the process of developing these remote sensing solutions, it has also been realized that multi- and hyper-spectral images have their own limitations (Heinzel and Koch 2012). For instance, the same tree species can have different spectral signatures in different parts of forest (Immitzer et al. 2012). Also, different tree species may possess similar spectra as well, particularly in a mixed pixel (Ghiyamat and Shafri 2010). Furthermore, multi- and hyper-spectral images are generally restricted to the horizontal plane, providing limited insight pertaining to the vertical profile of tree structure (Jones et al. 2010).

Recent developments in active remote sensing, particularly the light detection and ranging (LiDAR) technique, have shown great potential for tree species mapping due to its capability of capturing three-dimensional (3D) information of objects of interest (Brandtberg 2007; Clark et al. 2004; Coops et al. 2007; Holmgren and Persson 2004; Hyyppä et al. 2001; Lindberg et al. 2014; Næsset 2002). Unlike multi- and hyper-spectral images, it is possible to retrieve structural properties of trees from LiDAR, based on the discrete points or full-waveform data (Alonzo et al. 2014; Asner et al. 2008; Coops et al. 2007; Dalponte et al. 2014; Onojeghuo and Blackburn 2011; Shang and Chazette 2014). From a morphological point of view, tree species differ in their foliage distributions and branching patterns under different canopy conditions, resulting in diverge architectures which can be captured by LiDAR. For instance, the foliage of Norway spruce (Picea abies) 
(Fig. 2.1a) is clustered near the stem with pyramidal crown shape, while the foliage of European beech (Fagus sylvatica) (Fig. 2.1b) is more evenly distributed along the stem and has an oval crown shape. Histograms of laser pulse return frequency within varying height bins illustrate reflection allocation throughout the canopy (Fig. 2.1). A larger number of returns are reflected within the upper layer of spruce compared to beech. Under leaf-off condition, more returns were allocated towards the bottom of the canopy yet the top of the canopy was still well-represented by the LiDAR point cloud distribution (Fig. 2.1b). Thus, tree morphology characterized by LiDAR metrics may increase the ability to accurately discriminate tree species.
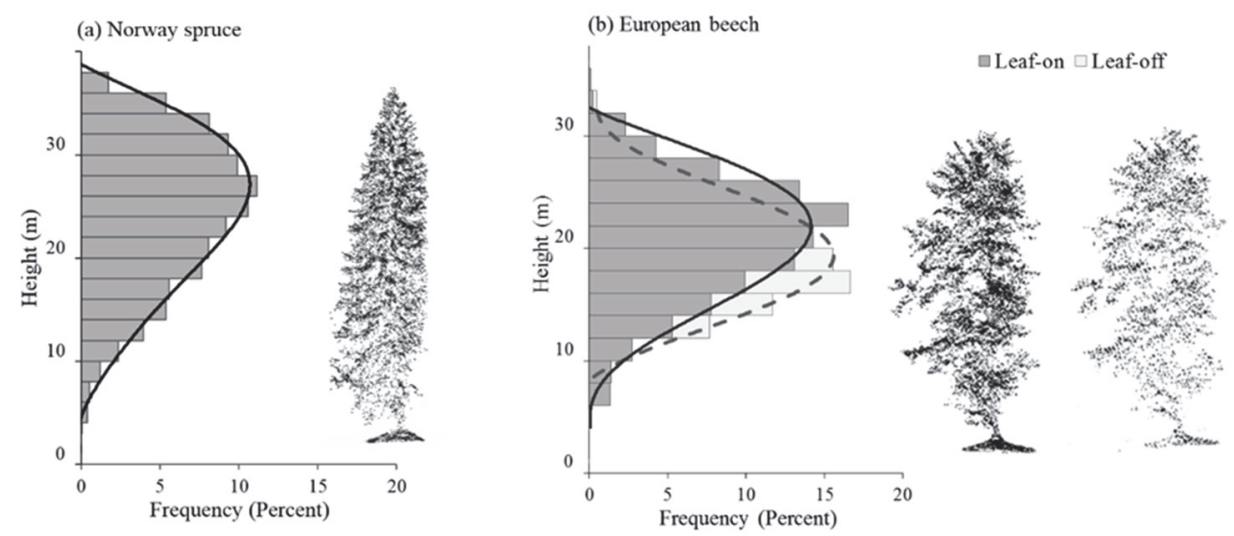

Fig. 2.1 Example of distributions of canopy laser pulse returns within (a) Norway spruce, and (b) European beech under leaf-on and leaf-off conditions using airborne LiDAR data.

Over the past decade, a large number of airborne LiDAR-derived metrics have been proposed for tree species classification (Brandtberg 2007; Brandtberg et al. 2003; Cao et al. 2016; Holmgren and Persson 2004; Hovi et al. 2016; Kim et al. 2011; Kim et al. 2009; Li et al. 2013; Lin and Herold 2016; Moffiet et al. 2005; Ørka et al. 2009; Reitberger et al. 2008). Generally, these LiDAR metrics can be categorized into two groups, namely geometric and radiometric metrics. The geometric metrics describe the geometric structure of trees (e.g. crown shape, tree height and crown volume) while the radiometric metrics refer to specific echo parameters that are extracted from the received waveform (e.g. the backscatter cross-section, the energy of laser points, and the distance between two waveform echoes) (Koenig and Höfle 2016; Wagner 2010). In particular, the intensity of the backscattered signal is related to foliage type, leaf size, leaf orientation, leaf clumping and foliage density (Kim et al. 2009; Korpela et al. 2010; Suratno et al. 2009). The echo width is dependent on the amount, distribution and orientation 
of scattering elements along the laser beam direction. These properties can all vary within and between tree species and thus may be useful for differentiating materials and ultimately tree species. Previous studies have demonstrated that LiDAR metrics can be used to improve the mapping accuracy of tree species. However, most of these studies focused on data-driven or algorithm-driven approaches and pursued an optimization of classification accuracy (Fassnacht $e t$ al. 2016). Consequently, an in-depth ecological and biological understanding of the linkage between tree species morphology and LiDAR derived metrics has not been performed. Identifying essential LiDAR metrics for tree species classification can not only reduce the redundant or overfitting caused by highly correlated metrics, but also help us build links between the inherent architectural differences of tree species and how they manifest in LiDAR metrics.

The phenological development of tree species is characterized by distinct seasonal phases of bud burst, leaf flushing, flowering, senescence and dormancy (Calle et al. 2010). The physical changes in canopy structure are particularly prominent for deciduous tree species. The integration of LiDAR data acquired under leaf-on and leaf-off conditions has been proven useful for tree species classification in previous studies (Kim et al. 2009; Ørka et al. 2010; Reitberger et al. 2008; Yao et al. 2012). Although some of these studies suggested several important LiDAR metrics for tree species classification, the majority of them focused on the effects of different canopy conditions on tree properties or only considered a few LiDAR metrics. The role of LiDAR metrics derived from both discrete point and full-waveform data under leaf-on and leaf-off conditions for individual tree species classification has not been explored. Moreover, Sumnall et al. (2016) concluded that the greatest complimentary information about a forest canopy profile can be derived from both leaf-on and leaf-off data rather than discrete return or full-waveform LiDAR data. Nonetheless, due to the incompatibility of LiDAR collections, data availability as well as the high costs associated with LiDAR acquisitions and data processing efforts, the full potential of multi-temporal LiDAR datasets for tree species classification has yet to be realized.

This study aims to evaluate the performance of 37 frequently used metrics derived from both discrete return and full-waveform airborne LiDAR data under leaf-on and leaf-off conditions, respectively, for discriminating six different tree species in a mixed temperate forest in Germany. Specifically, we set out to: (1) evaluate the correlation among those commonly used LiDAR metrics, (2) assess the performance of LiDAR metrics for tree species classification under leaf-on and 
leaf-off conditions and select important input metrics, and (3) identify the most important LiDAR metrics for discriminating tree species and understand how they are linked with tree species morphology.

\subsection{Materials and Methods}

\subsubsection{Study area and tree species}

The study area is located in the Bavarian Forest National Park $\left(49^{\circ} 3^{\prime} 19^{\prime \prime} \mathrm{N}\right.$, $13^{\circ} 12^{\prime} 9^{\prime \prime}$ E), a mixed temperate forest situated in the south-eastern part of Germany (Fig. 2.2). The park covers a total area of 24218 hectares with an elevation ranging from approximately $600 \mathrm{~m}$ to $1452 \mathrm{~m}$. The forest is dominated by Norway spruce (Picea abies), which co-habits with European beech (Fagus sylvatica) on the slopes, and silver fir (Abies alba) at low and intermediate elevations. Pioneer deciduous species are also present such as white birch (Betula pendula), sycamore maple (Acer pseudoplatanus), common rowan (Sorbus aucuparia), European ash (Fraxinus excelsior), European aspen (Populus tremula) and Field elm (Ulmus minor). However, they only represent $3.3 \%$ of the total basal area of the park (Cailleret et al. 2014).

We identified two species-rich sites within the park (Fig. 2.2) and used them as pilot study areas of interests. Each pilot site is approximately 25 hectares $(500 \mathrm{~m}$ $\times 500 \mathrm{~m}$ ). Detailed information about the two study sites, including topographic condition, soil type, tree density, tree height, forest types and stand age classes are provided in Table 2.1. The spatial location of individual tree species was collected with a Leica Viva GS10 Plus differential GPS (Leica Geosystems AG, Heerbrugg, Switzerland) in July 2016 and July 2017, respectively. The GPS data were post-processed to obtain differentially corrected coordinates with an accuracy less than $0.25 \mathrm{~m}$. In total, we have collected 256 locations of trees at site A and 193 locations of trees at site B. 


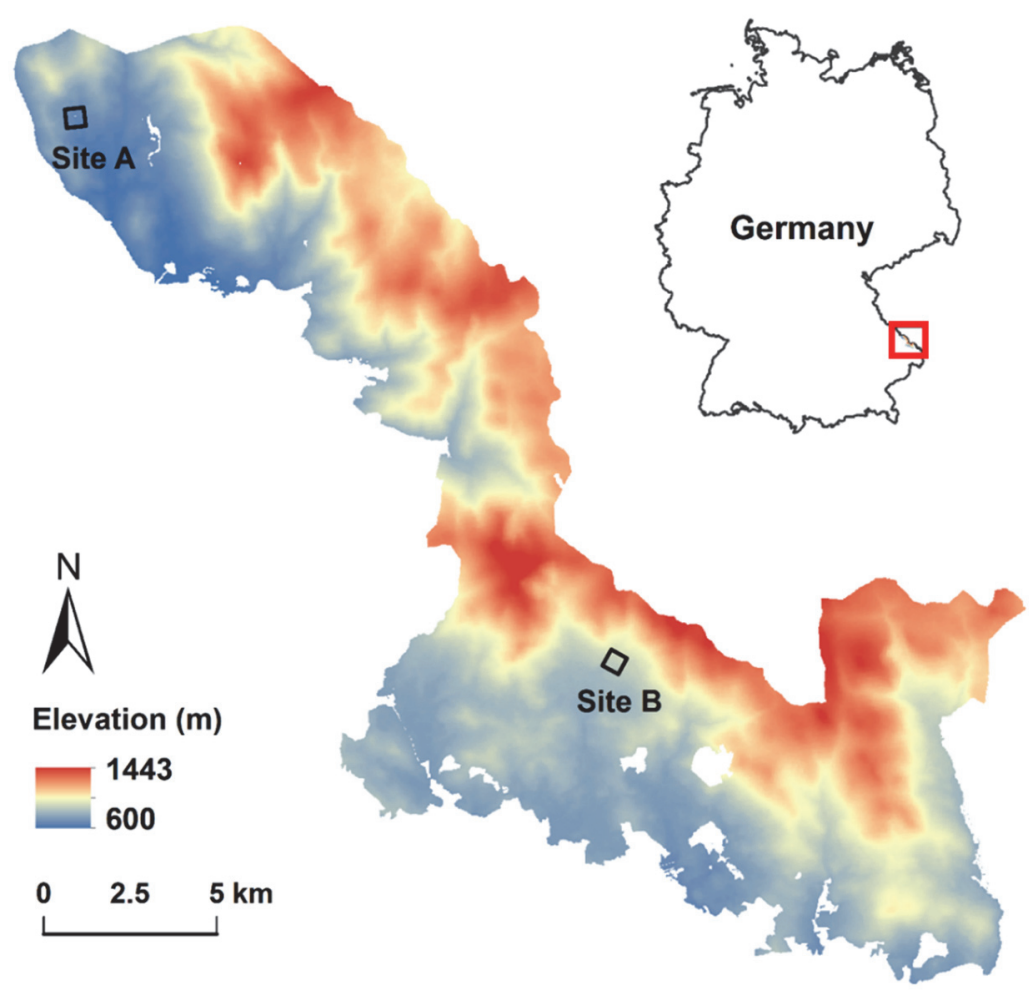

Fig. 2.2 The study area in Germany and the location of the two pilot study sites in the Bavarian Forest National Park

Table 2.1 Characteristics of the two pilot study sites

\begin{tabular}{lll}
\hline & Site A & Site B \\
\hline Size (ha) & 25 & 25 \\
Elevation (m) & $675-732$ & $845-906$ \\
Slope (degree) & $9.12 \pm 5$ & $8.76 \pm 3$ \\
Soil type & Brown forest soils & Loose brown soils \\
& and peat soils & and gley soils \\
Forest type and stand age & Mature coniferous & Mature deciduous \\
classes & and mixed stands & and mixed stands \\
Tree density (per ha) & 445 & 458 \\
\hline
\end{tabular}




\subsubsection{Airborne LiDAR data collection and processing}

The airborne LiDAR data were acquired on 11 April and 18 August 2016 under leaf-off and leaf-on canopy conditions respectively, using a Riegl LMS-Q680i scanner (wavelength $1550 \mathrm{~nm}$ ) integrated in a full-waveform laser scanning system. The two datasets were collected with the same sensor and same settings. The mean flight speed was $50 \mathrm{~ms}^{-1}$ and the flying altitude was approximately 300 $\mathrm{m}$ above ground. The pulse repetition frequency was $400 \mathrm{kHz}$ with a scan angle around $\pm 15^{\circ}$. The point density was around $70 \mathrm{pts} / \mathrm{m}^{2}$. A total of 21 flight lines were recorded with $50 \%$ strip overlap.

Both point cloud data from Gaussian decomposition (Wagner et al. 2006) and raw full-waveform data were delivered by the Milan Flug GmbH Company. The point cloud information contains 3D coordinates ( $\mathrm{x}, \mathrm{y}$ and $\mathrm{z}$ ), intensity, return number, number of returns, echo width and the GPS timestamp of the return. The extracted point clouds and associated waveforms were stored in the LAS 1.2 format. We used the LAStools software package (LAStools, version 160921, rapidlasso $\mathrm{GmbH}$, http://lastools.org) to create the $0.5 \mathrm{~m}$ resolution digital terrain models (DTM) from the LiDAR data. We normalized the height of each LiDAR return to height above ground by subtracting the elevation of the DTM below each point. It should be noted that we have used the same sensor and same settings, calibrating the intensity data of the two LiDAR datasets is not required in this study as suggested by Korpela et al. (2010). In addition, since the altitude of the two LiDAR flights were the same, and our two pilot study sites were topographically similar (having a gentle slope), the intensity normalization for the range between the sensor and object as well as for the incidence angle were therefore ignored (Vain and Kaasalainen 2011).

\subsubsection{Individual tree segmentation}

An adapted 3D segmentation algorithm proposed by Yao et al. (2013) was used to automatically extract individual trees in this study. It is an object-based point cloud analysis approach for tree detection and uses normalized cut segmentation as the core part of the method. The $3 \mathrm{D}$ segmentation algorithm is a two-tiered procedure. The steps of the entire procedure are as follows: (i) decomposition of full-waveform data; (ii) local tree maxima filtering; (iii) mean shift clustering; (iv) feature derivation for mean shift clusters; (v) normalized cut segmentation; and (vi) height filtering of the segmentation results. 
We chose the sample trees and linked them to the correct LiDAR segmentation results for analysis by conducting the following procedures: (1) we first overlaid the location of sample trees with the 3D segmentation results and the aerial photograph (spatial resolution $0.25 \mathrm{~m}$ ); (2) we then visually verified each sample tree based on the additional information we have recorded in the field (e.g. photos of the sample trees and the species of surrounding trees) as well as the crown shape interpreted from the very high resolution aerial photograph, and tried to connect it with the 3D LiDAR segments; (3) if a sample tree was not detected by the segmentation algorithm, it was removed from further analysis; (4) if a sample tree was assigned to more than one tree position, it was also removed from further analysis. The LiDAR points of each tree segment were extracted and assigned to the corresponding sample trees for both sites A and B. As a result, only 205 sample trees from site A and 158 sample trees from site B were selected and used for the current study. The details of the number and the mean height of each tree species are shown in Table 2.2.

Table 2.2 The sample size, mean height and standard deviation (SD) of each tree species in site A and site B

\begin{tabular}{lllll}
\hline Tree & $\begin{array}{l}\text { Site A } \\
\text { Sample } \\
\text { species }\end{array}$ & $\begin{array}{l}\text { Mean height and } \\
\text { size }\end{array}$ & $\begin{array}{l}\text { Site B } \\
\text { Sample }\end{array}$ & $\begin{array}{l}\text { Mean height and } \\
\text { size }\end{array}$ \\
\hline Beech & 39 & $25.9 \pm 7.2$ & 29 & $26.4 \pm 5.0$ \\
Birch & 36 & $18.2 \pm 7.5$ & 29 & $18.6 \pm 4.8$ \\
Fir & 31 & $35.7 \pm 6.7$ & 21 & $27.9 \pm 7.7$ \\
Maple & 40 & $20.7 \pm 6.1$ & 37 & $22.3 \pm 7.0$ \\
Rowan & 21 & $17.5 \pm 6.3$ & 18 & $16.7 \pm 7.2$ \\
Spruce & 38 & $28.3 \pm 8.0$ & 24 & $30.7 \pm 6.7$ \\
\hline
\end{tabular}

\subsubsection{Derivation of LiDAR metrics under leaf-on and leaf-off conditions}

The most commonly used LiDAR metrics describing tree height is the percentile of the height distribution of laser pulses (Koenig and Höfle 2016; Li et al. 2013; Lin and Hyyppä 2016; Ørka et al. 2009; Sumnall et al. 2016; Vauhkonen et al. 2010b). The lower limit of the canopy was defined by a threshold value of $2 \mathrm{~m}$. Separate distributions were created for the first and last returns recorded as the percentage of first return to all returns and the percentage of last returns to all returns. From the echo height distribution we computed the maximum, mean, standard deviation, coefficient of variation, kurtosis, skewness, and height percentiles at 5\% intervals $(\mathrm{Hp} 5, \mathrm{Hp} 10, \ldots, \mathrm{Hp} 90, \mathrm{Hp} 95)$ of tree height within a 
tree segment (Andersen et al. 2005; Hopkinson et al. 2006; Lin and Hyyppä 2016; Muss et al. 2011; Næsset 2002; Naidoo et al. 2012; Vauhkonen et al. 2010a). Due to a strong correlation among the height percentile metrics between every $5 \%$ intervals, we selected the Hp25 and Hp90 percentiles for further analysis. The commonly used tree crown related LiDAR metrics include crown base height, crown volume and crown area which were extracted based on the methods proposed by Yao et al. (2012). The ratio of crown base height to tree height and the ratio of crown volume to crown area were added to reduce the impacts of different tree ages on tree species classification. Moreover, the mean height of first-or-single returns and the mean height of single returns were generated as descriptions to understand how crown shape reflected the different return types of laser pulses. As species identification using 3D features should be based on the architecture of the tree, not on its size, height related LiDAR metrics (i.e. the mean value of height, the standard deviation of height, the height percentiles, the mean height of first-or-single returns and the mean height of single returns) were generated based on normalized heights to eliminate scale dependency. The normalized height is the height of each return above ground divided by the height of the tree it belongs to. In total, 16 geometric metrics were generated as listed in Table 2.3.

The radiometric metrics used in the tree species classification were derived from the intensity and echo width distributions. In addition to the $25 \%$ and $90 \%$ percentiles of the intensity, the maximum, mean, standard deviation, coefficient of variation, skewness and kurtosis of intensity within a tree segment were also computed (Dalponte et al. 2008; Heinzel and Koch 2011; Hovi et al. 2016; Korpela et al. 2010; Lin and Hyyppä 2016; Ørka et al. 2009; Yao et al. 2012). Similarly, the $25 \%$ and $90 \%$ percentiles of the echo width, the maximum, mean, standard deviation, coefficient of variation, skewness, and kurtosis of echo width within a tree segment were derived from full-waveform data as the radiometric parameters (Heinzel and Koch 2011; Höfle et al. 2012; Hovi et al. 2016; Lin 2015; Yao et al. 2012). Additionally, intensity and echo width with respect to two different echo categories: "first-or-single returns" and "single returns" (i.e. Imean_first and Imean_single for intensity, EWmean_first and EWmean_single for echo width) were also generated from each segment (Hovi et al. 2016; Ørka et al. 2010). In total, 21 radiometric metrics have been generated as listed in Table 2.3. All metrics were generated under both leaf-on and leaf-off conditions with the R statistical language version 3.3.3 (http://www.r-project.org/). 
Table 2.3 Description of the 37 generated LiDAR metrics

\begin{tabular}{|c|c|c|c|}
\hline Metrics & Definition & Metrics & Definition \\
\hline \multicolumn{2}{|l|}{ Geometrics } & \multicolumn{2}{|l|}{ Radiometrics } \\
\hline Hmax & Maximum height & $\operatorname{Imax}$ & Maximum intensity \\
\hline Hmean & Mean height & Imean & Mean intensity \\
\hline Hsd & Standard deviation of height & Isd & Standard deviation of intensity \\
\hline $\mathrm{Hcv}$ & Coefficient variation of height & Icv & Coefficient variation of intensity \\
\hline Hkurt & Kurtosis of height & Ikurt & Kurtosis of intensity \\
\hline Hskew & Skewness of height & Iskew & Skewness of intensity \\
\hline Нp25 & 25 th percentile of heights & Ip25 & 25 th percentile of intensity \\
\hline Нp90 & 90th percentile of heights & Ip90 & 90th percentile of intensity \\
\hline Hmean_first & $\begin{array}{l}\text { Mean height of first-or-single } \\
\text { returns }\end{array}$ & Imean_first & $\begin{array}{l}\text { Mean intensity of first-or-single } \\
\text { returns }\end{array}$ \\
\hline Hmean_single & Mean height of single returns & Imean_single & Mean intensity of single returns \\
\hline \multicolumn{2}{|c|}{ First:total_returns Percentage of first returns above $2 \mathrm{~m}$} & Ewmin & Minimum echo width \\
\hline \multicolumn{2}{|c|}{ Last:total_returns Percentage of last returns above $2 \mathrm{~m}$} & Ewmax & Maximum echo width \\
\hline All_returns & All returns above $2 \mathrm{~m}$ & Ewmean & Mean echo width \\
\hline $\mathrm{CBH}: \mathrm{H}$ & Ratio of crown base height to height & Ewsd & Standard deviation of echo width \\
\hline C_volume:area & $\begin{array}{l}\text { Ratio of crown volume to crown } \\
\text { area }\end{array}$ & Ewcv & Coefficient variation of echo width \\
\hline \multirow[t]{6}{*}{ CNR } & Canopy relief ratio & Ewkurt & Kurtosis of echo width \\
\hline & & Ewskew & Skewness of echo width \\
\hline & & Ewp25 & 25 th percentile of echo width \\
\hline & & Ewp90 & 90th percentile of echo width \\
\hline & & Ewmean_first & $\begin{array}{l}\text { Mean echo width of first-or-single } \\
\text { returns }\end{array}$ \\
\hline & & Ewmean_single & Mean echo width of single returns \\
\hline
\end{tabular}

\subsubsection{Correlation analysis of LiDAR metrics}

LiDAR metrics can be useful in species classification if they differ significantly between species (Brandtberg et al. 2003; Holmgren and Persson 2004). From a statistical point of view, metric selection can reduce the number of input variables which is important for building efficient, stable and transferable classification models. In this study, 37 geometric and radiometric metrics (under both leaf-on and leaf-off conditions) were analysed to reduce the collinearity and avoid overfitting during the tree species classification process (Table 2.3). The Pearson's Correlation Coefficient was used to examine the correlations between LiDAR metrics for both the leaf-on and leaf-off datasets. Here, we used the threshold of correlation coefficients between LiDAR metrics of $|r|<0.70$, as such a threshold has been proved an appropriate indicator for when collinearity begins to severely distort model estimation and subsequent prediction (Dormann et al. 2013). 


\subsubsection{Random Forest algorithm}

Random Forest is a popular and powerful machine learning algorithm (Belgiu and Drăguţ 2016; Fassnacht et al. 2016; Ørka et al. 2010; Vauhkonen et al. 2010a). We used the Random Forest algorithm, with internal cross-validation, to assess the performance of "selected metrics" for tree species discrimination for leaf-on, leaf-off and combined datasets. During Random Forest classification, approximately one-third of the samples were left out of the input training dataset as "out-of-bag" (OOB) samples to estimate the classification error and derive variable importance. The Mean Decrease Accuracy (MDA) index, which quantifies the degree to which inclusion of a variable in the model decreases the mean squared error, was used to assess the variable importance for classification (Breiman 2001; Liaw and Wiener 2002). The mean decrease in accuracy of a variable is determined during the out of bag error calculation phase. The more the accuracy of the random forest decreases due to the exclusion (or permutation) of a single variable, the more important that variable is deemed, and therefore variables with a large mean decrease in accuracy are more important for classification (Breiman 2001; Teicher et al. 2012).

The Random Forest algorithm has several advantages with respect to the current assessment of LiDAR metrics derived from different acquisitions in tree species classification, e.g. (1) it can handle a large number of input variables without variable deletion; (2) it computes an error matrix based on an internal validation process; (3) it estimates which variables are important in the classification, measured as the mean decrease accuracy; (4) the generated forests can be saved for future use on other data; and last but not least (5) it reduces overfitting and is therefore more accurate than equivalent discriminative, or boosted regression based methods trained on the same data (Cootes et al. 2012). However, similar to most classifiers, Random Forest algorithm can also suffer from the curse of learning from an extremely imbalanced training dataset (Chen et al. 2004). Hence, we used the Random Forest algorithm to derive both classification accuracy as well as the variable importance in this study. The classification was carried out with the R package "randomForest" (Liaw and Wiener 2002). We tested different values for the Random Forest parameter "Ntree" (i.e. number of trees grown) and parameter "Mtry" (i.e. number of predictors sampled for splitting at each node), varying in each test from 1 to 500 and from 1 to 30 , respectively, and we set up a loop to run the Random Forest algorithm for each combination of parameters and chose the model with the best classification performance. Then we applied this model in study site B for classification, accuracy assessment and metrics 
evaluation using samples independent of the samples used to develop the model in study site A, and assessed the robustness and transferability of "selected metrics" by comparing the importance of selected metrics.

\subsubsection{Classification accuracy assessment}

We used producer's accuracy, user's accuracy, overall accuracy and kappa coefficient to assess the classification results (Cohen 1960). We also used the McNemar's test to determine whether statistically significant differences exist between classifications (de Leeuw et al. 2006; McNemar 1947).

Using Random Forest we iterated with the 12 metrics generated during the first step of metrics selection under leaf-on and leaf-off conditions using 205 sample trees in study site A. The performance of the LiDAR metrics was further verified by 158 independent sample trees collected in study site B by using the Random Forest model established in study site A. The classification results and metrics importance derived from site B were compared to the results of site A under all leaf-on, leaf-off and integration conditions.

\subsubsection{Determining the importance of the LiDAR metrics}

We calculated classification accuracy and evaluated metrics importance under leaf-on and leaf-off conditions. For each condition, we chose the 7 top-ranked metrics (14 metrics in total) as the input of final classification. We selected 14 metrics because inclusion of more features did not increase the classification accuracy significantly, which was also demonstrated by Li et al. (2013). After selection of important LiDAR metrics under leaf-on and leaf-off conditions, we input the 14 top-ranking metrics as the integration metrics of leaf-on and leaf-off conditions for the tree species classification. We applied the classification model with the same parameter settings to study site B, and recorded the performance of classification and the importance of input metrics. Then we identified the most consistently significant metrics and evaluated the contribution of each metric based on the classification results.

\subsection{Results}

\subsubsection{Correlation of LiDAR metrics}

High correlation $(|r|>0.7)$ was observed among many of the geometric and radiometric metrics derived from both leaf-on and leaf-off conditions (Fig. 2.3). For the geometric metrics, high correlation was found between the height related 
metrics such as Hmax, Hmean, Hsd, Hcv, Hskew, Hp25, Hp90, Hmean_first, Hmean_single and CNR. For radiometric metrics, the echo width related metrics presented stronger correlations between the metrics in comparison to intensity related metrics. Specifically, three echo width related metrics, i.e. Ewmean, Ewsd and Ewcv were highly correlated to each other under both leaf-on and leaf-off conditions. As a result, only 30 out of 74 LiDAR metrics (combining both leafon and leaf-off conditions) were found with an absolute correlation coefficients less than 0.70 .

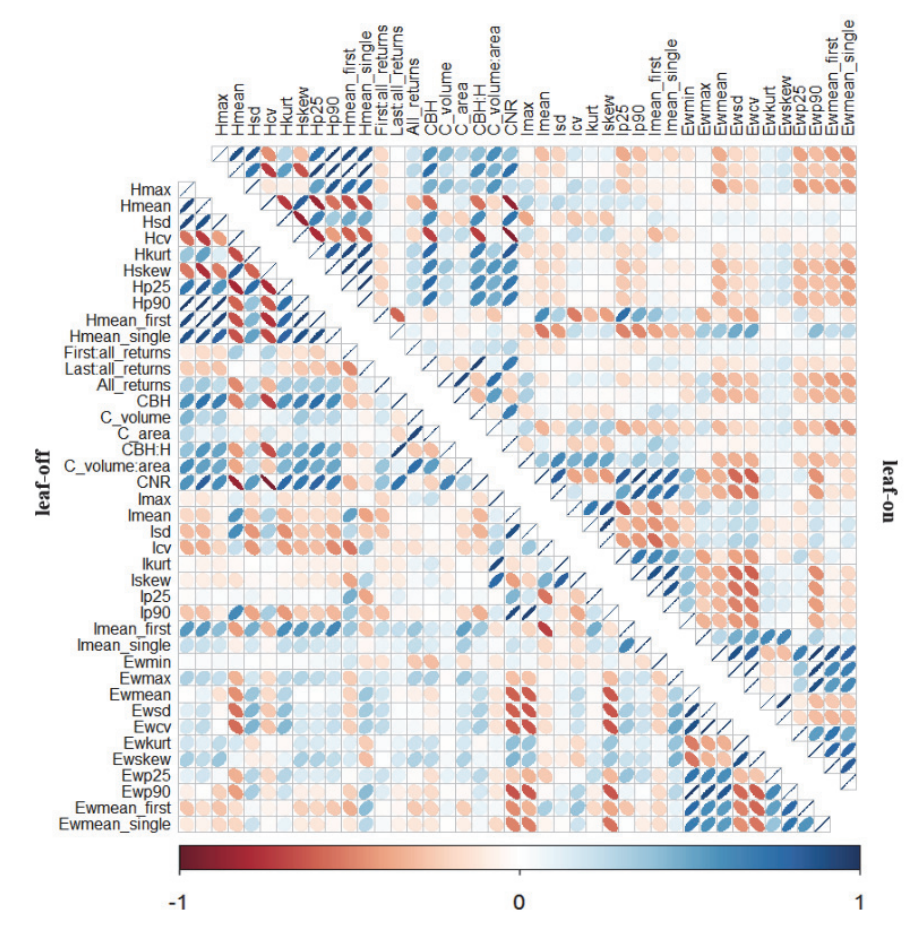

Fig. 2.3 Cross-correlation matrix of the $37 \mathrm{LiDAR}$ metrics derived under leaf-off (lower) and leaf-on (upper) conditions. Blue colours indicate positive correlations and red colours indicate negative correlations. See Table 2.3 for definitions of the metrics.

\subsubsection{LiDAR metrics selection}

Firstly, we evaluated 37 LiDAR metrics (under both leaf-on and leaf-off condition) based on correlation coefficients as well as the ranking of their importance using the Mean Decrease Accuracy index (MDA). We retained the metrics with a correlation coefficient $|r|<0.70$ and the higher ranked metric when two compared metrics had $|r|>0.70$. Then, we used 12 top-ranked significant metrics as the input for further classification (Table 2.4). Finally, we selected the 
top 7 metrics from each condition (i.e. leaf-on and leaf-off) as the final input metrics for classification based on MDA (Table 2.4).

Table 2.4 Selected LiDAR metrics derived under leaf-on and leaf-off conditions and their Mean Decrease Accuracy (MDA). The top 7 metrics indicated by the asterisk (*) from each condition (i.e. leaf-on and leaf-off) were used as final input metrics for tree species classification.

\begin{tabular}{llllll}
\hline Leaf-on & MDA & Leaf-off & MDA & Leaf-on and leaf-off & MDA \\
\hline *Imean_first & 14.221 & *Imean_first & 13.185 & Imean_first_off & 13.01 \\
*Ewmean & 12.185 & *Hmean_single & 11.796 & Hmean_single_off & 12.64 \\
*Hmax & 11.530 & *Ewmean & 10.471 & Imean_first_on & 11.15 \\
*Imean_single & 9.117 & *Icv & 10.313 & Icv_off & 10.64 \\
*Imean & 8.551 & *Ewmean_first & 10.024 & Ewmean_on & 10.11 \\
*Hmean_single & 8.386 & *Hmean_first & 9.256 & Hmax_on & 10.09 \\
*Ewmean_first & 8.071 & *Hmean & 9.021 & Ewmean_off & 9.83 \\
Ewmean_single & 7.623 & Hcv & 8.309 & Ewmean_first_on & 9.15 \\
Hmean_first & 7.220 & Ewp90 & 8.249 & Ewmean_first_off & 9.13 \\
Ip90 & 6.844 & Hp25 & 7.881 & Hmean_single_on & 8.95 \\
Hp90 & 3.698 & Ewmean_single & 6.822 & Imean_on & 8.92 \\
C_volume:area & 1.172 & Hkurt & 5.587 & Hmean_first_off & 8.13 \\
& & & & Imean_single_on & 7.11 \\
& & & & Hmean_off & 5.88 \\
\hline
\end{tabular}

\subsubsection{Comparison of classification accuracies}

The classification results produced by the Random Forest algorithm are presented in Table 2.5. It is shown that there was no statistically significant difference in tree species mapping accuracy between the use of leaf-on and leaf-off LiDAR metrics (McNemar's test, $p>0.05$ ) (Table 2.6). However, combining leaf-on and leaf-off LiDAR metrics significantly increased the overall accuracy from 58.2\% (leaf-on) and $62.0 \%$ (leaf-off) to $66.5 \%$ as well as the kappa coefficient from 0.47 (leaf-on) and 0.51 (leaf-off) to 0.58 (McNemar's test, $p<0.05$ ) (Table 2.6).

We executed the selected LiDAR metrics in study site B in order to assess the robustness and transferability of the selected metrics. The difference in the classification performance was minor between leaf-on and leaf-off conditions, while combining LiDAR metrics derived under leaf-on and leaf-off conditions significantly improved the accuracy (Table 2.6). Both the classification in site A and site $\mathrm{B}$ generated modest and comparable classification accuracy. For individual tree species, beech, birch and spruce were classified with higher user's and producer's accuracy under leaf-off rather than leaf-on condition (Table 2.5). 
Rowan was misclassified under leaf-on and leaf-off conditions using selected metrics, and only a slight improvement occurred using the combined leaf-on and leaf-off datasets.

Table 2.5 Comparison of overall accuracy and kappa coefficient for tree species classification using leaf-on, leaf-off and combination of leaf-on and leaf-off LiDAR metrics.

\begin{tabular}{|c|c|c|c|c|c|c|c|c|c|c|c|c|}
\hline \multirow{3}{*}{ Tree species } & \multicolumn{6}{|l|}{ Site A } & \multicolumn{6}{|l|}{ Site B } \\
\hline & \multicolumn{2}{|l|}{ Leaf-on } & \multicolumn{2}{|c|}{ Leaf-off } & \multicolumn{2}{|c|}{ Integration } & \multicolumn{2}{|c|}{ Leaf-on } & \multicolumn{2}{|c|}{ Leaf-off } & \multicolumn{2}{|c|}{ Integration } \\
\hline & $\mathrm{UA}(\%)$ & PA(\%) & $\mathrm{UA}(\%)$ & $\mathrm{PA}(\%)$ & $\mathrm{UA}(\%)$ & PA(\%) & $\mathrm{UA}(\%)$ & PA(\%) & $\mathrm{UA}(\%$ & ) PA(\%) & $\mathrm{UA}(\%$ & $\mathrm{PA}(\%)$ \\
\hline Beech & 54.1 & 57.1 & 67.6 & 67.6 & 64.9 & 64.9 & 48.3 & 50.0 & 58.6 & 28.6 & 55.2 & 55.2 \\
\hline Birch & 54.5 & 50.0 & 57.6 & 57.6 & 63.6 & 58.3 & 75.9 & 73.3 & 79.3 & 76.7 & 82.8 & 82.8 \\
\hline Maple & 68.9 & 60.8 & 66.7 & 63.3 & 64.4 & 65.9 & 67.6 & 61.0 & 70.3 & 61.9 & 78.4 & 65.9 \\
\hline Rowan & 26.3 & 38.5 & 21.5 & 40.0 & 36.8 & 63.6 & 31.3 & 16.7 & 27.8 & 50.0 & 33.3 & 46.2 \\
\hline Fir & 66.7 & 57.1 & 66.7 & 60.6 & 73.3 & 68.8 & 52.4 & 61.1 & 61.9 & 56.5 & 57.1 & 70.6 \\
\hline Spruce & 56.1 & 65.7 & 61.0 & 56.8 & 65.9 & 60.0 & 58.3 & 58.3 & 70.8 & 68.0 & 75.0 & 69.2 \\
\hline $\mathrm{OA}$ & $57.1 \%$ & & $60.0 \%$ & & $62.4 \%$ & & $58.2 \%$ & & $62.0 \%$ & & $66.5 \%$ & \\
\hline Kappa & 0.46 & & 0.49 & & 0.54 & & 0.47 & & 0.51 & & 0.58 & \\
\hline
\end{tabular}

OA: overall accuracy; UA: user's accuracy; PA: producer's accuracy

Table 2.6 McNemar's test for pairwise comparison between classification results derived from the three datasets (leaf-on, leaf-off, and integration). The number in the table is $p$ value. The number with an asterisk $\left(^{*}\right)$ indicates that the difference between classifications is significant at a $5 \%$ significant level.

\begin{tabular}{lllll}
\hline & Site A & \multicolumn{3}{c}{ Site B } \\
\hline & Leaf-off & Integration & Leaf-off & Integration \\
Leaf-on & 0.16 & $<0.01 *$ & 0.11 & $<0.01^{*}$ \\
Leaf-off & & 0.09 & & $0.02^{*}$ \\
\hline
\end{tabular}

\subsubsection{Performance of LiDAR metrics in tree species classification}

Fig. 2.4 presents the relative importance and ranking of the selected LiDAR metrics for tree species classification for the two pilot study sites based on leafon, leaf-off and their combination. It indicates that the significant metrics and their ranks vary under different conditions. However, Imean_first appeared as the first-ranked metrics under every condition in both sites A and B. When metrics selected from leaf-on and leaf-off datasets were combined, 4 out of 5 top ranked metrics were the same as those derived in site A (Fig. 2.4c, 2.4f), which were all radiometric metrics. Hmean_single had a performance comparable to the Imean_first under leaf-off and combination conditions in site A, however, it did not show superior performance in the classification of site B. Comparing 
important metrics under each condition, Imean_first and EWmean consistently appeared as top 5 metrics through different canopy conditions.

We also tested the accumulated contribution rate increased through increasing the number of selected metrics for classification. Adding LiDAR metrics produced the largest increase in the classification contribution rate, from $65 \%$ to $100 \%$, reached the first peak (96.2\%) using the top 5 metrics, and stabilizing around $93 \%$ at 10 metrics, which supporting the choice of 10 features as a reasonable limit for species classification. The first 5 metrics were selected as the best performed for the separation of the tree species of interest according to the MDA in Random Forest that globally maximize the fitness function through iterations.

\subsubsection{The capability of metrics for tree species discrimination}

The differences between the six tree species for the top 4 ranked metrics are plotted in Fig. 2.5. It can be observed that the ability of the 4 most important metrics for tree species discrimination varies for each species under different conditions. It is evident that coniferous trees have a higher value of mean intensity of first returns compared to deciduous trees under leaf-off condition, while the difference of this metric between coniferous trees and deciduous trees becomes smaller under leaf-on condition (Fig. 2.5a). A similar pattern can be observed in Fig. $2.5 \mathrm{~d}$ about the normalized mean height of single returns. Fig. $2.5 \mathrm{c}$ shows a distinct difference of coefficient variation of intensity between leaf-on and leafoff conditions among 6 species, which differentiates coniferous trees from deciduous trees under leaf-off condition. Similarly, the mean value of echo width shows a superior ability to separate birch from other 5 tree species, especially under leaf-on condition (Fig. 2.5b). 

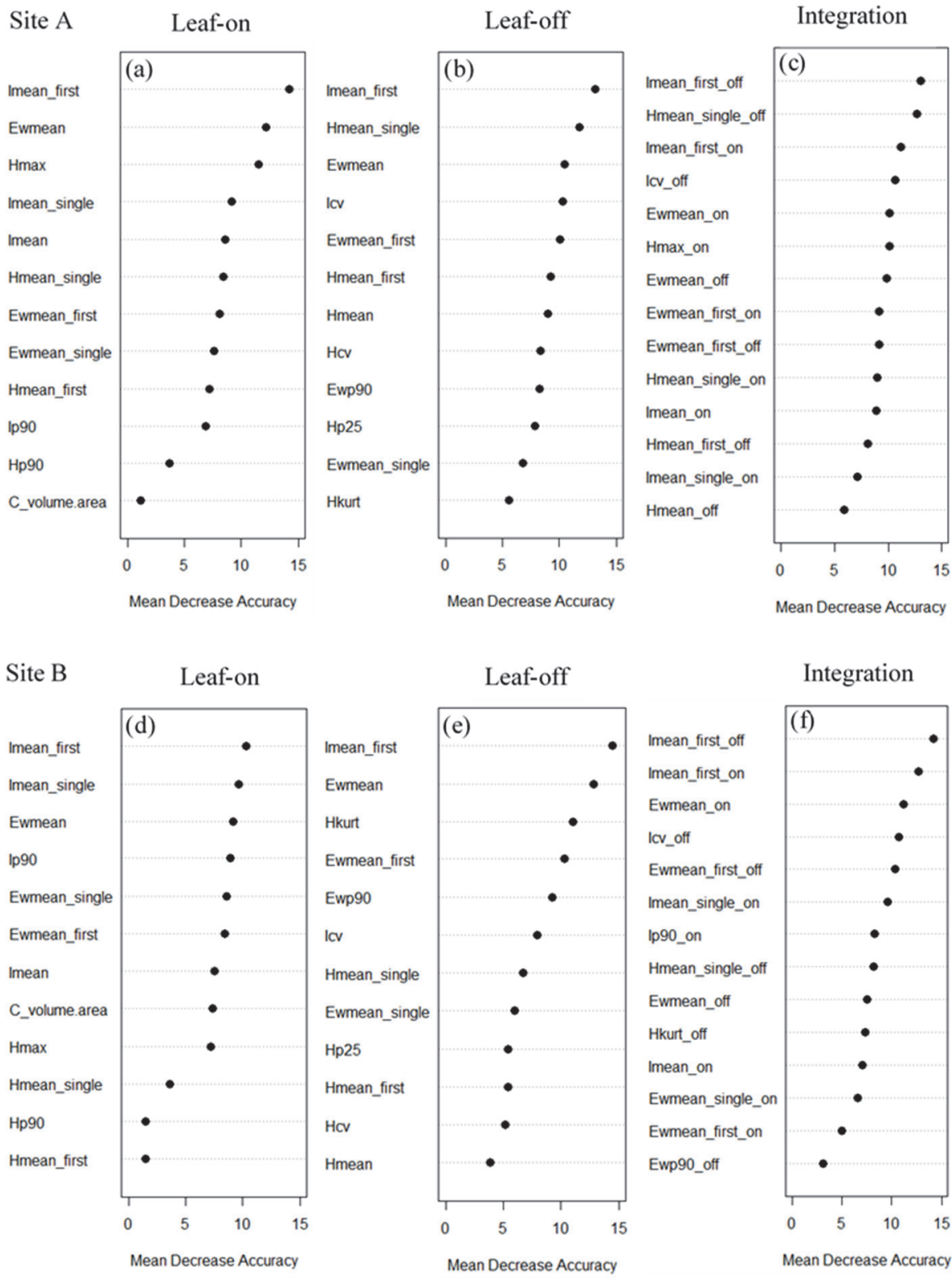

Fig. 2.4 The relative importance and ranking of the selected LiDAR metrics for tree species classification under different conditions derived from the pilot study site A and site B. The Mean Decreasing Accuracy indicates importance by how much the permutation (effective elimination) of a given variable decreases the accuracy of the overall fit. Metrics that are associated with the greatest decrease in accuracy coefficient following permutation are the most important. 

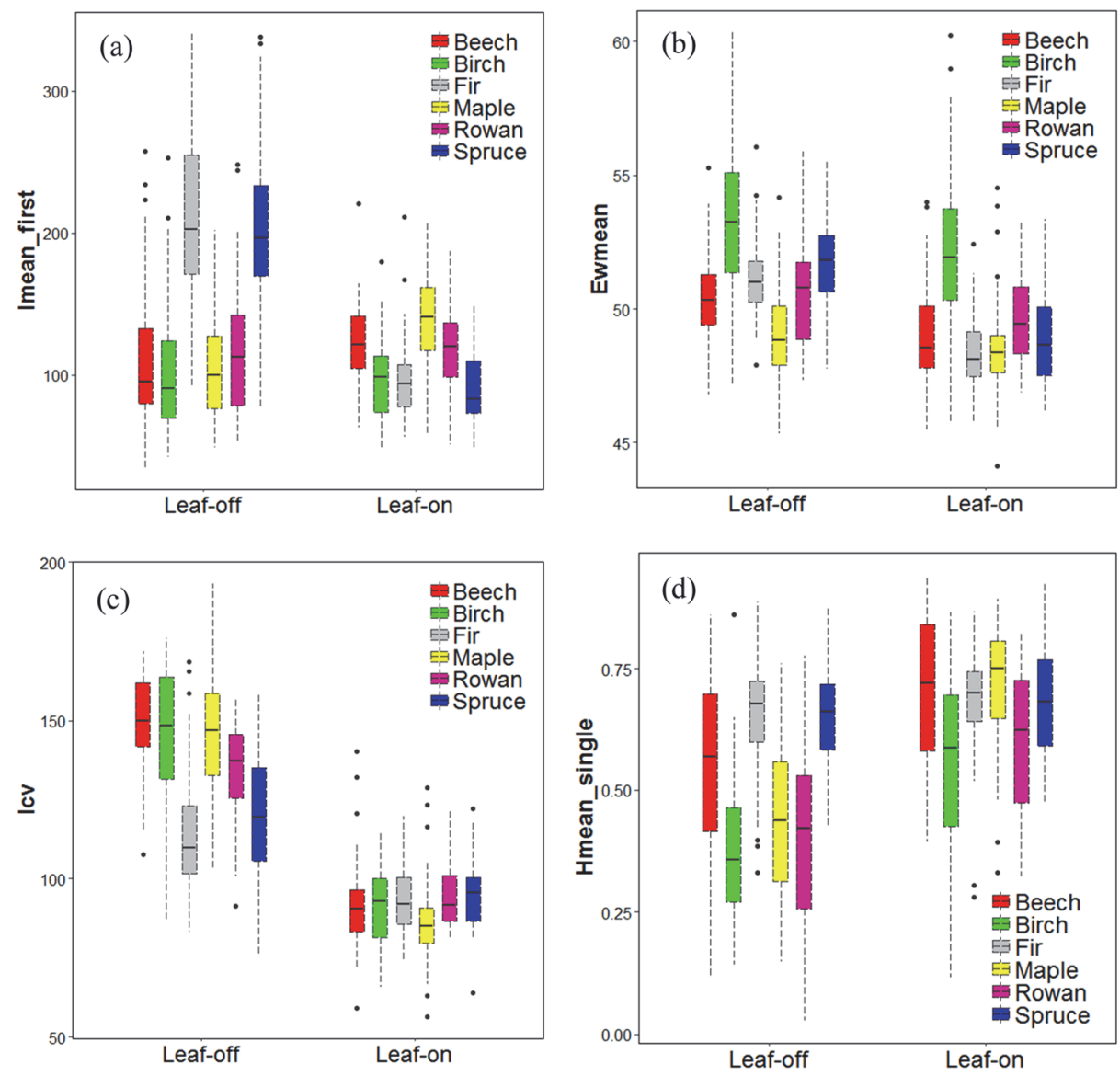

Fig. 2.5 Box plots of mean intensity of first-or-single returns (a), mean value of echo width (b), coefficient variation of intensity (c), and normalized mean height of single returns (d) among six tree species under leaf-on and leaf-off conditions. The value of each metrics is based on the sample trees from both site A and site B.

\subsection{Discussion}

In this study, we generated a set of geometric and radiometric metrics from airborne LiDAR data under leaf-on and leaf-off conditions and evaluated their performance for individual tree species discrimination in a mixed temperate forest in Germany. Our results demonstrated that radiometric features consistently contributed a higher accuracy compared to geometric features for classifying tree species under both leaf-on and leaf-off conditions. Specifically, the mean intensity of first-or-single returns as well as the mean value of echo width were identified as the most robust LiDAR metrics for discriminating six typical tree species in Central Europe. The importance of features derived from 
the first and the last returns for tree species classification was reported by Ørka et al. (2010) and the current study specified and verified this result. We found that the mean intensity of first-or-single returns was least sensitive to the canopy condition and well represented the structural and morphological characteristics of trees, which contributed most to the accuracy of tree species classification. Both the mean intensity of first-or-single returns and echo width are metrics which contain radiometric and geometric (3D) information. The mean intensity of first-or-single returns is derived from the outer "crown shell", which means narrow and peaked branch tips result in an increased proportion of partial hits by footprint (Korpela et al. 2013). It also implies that the difference in the spatial distribution of branches between different tree species is most prominent at the top of canopy. The major structural differences among tree species that are reflected in LiDAR metrics generally occur at the top crown layers rather than middle and low stem layers, which explained why radiometric metrics played an important role in species discrimination in comparison geometric metrics. However, the value of the mean intensity of first-or-single returns of deciduous trees in our study did not show a significant difference between leaf-off and leafon conditions (Fig. 2.5a) - this may be due to an earlier spring leaf unfolding phenology that occurred in 2016 in our study area. Contradictory to the study conducted by Sumnall et al. (2016), where echo width metrics were considered relatively unimportant for forest inventory purposes, we found that the mean echo width within a tree segment led to more stable performance than geometric features, with an important contribution from the intensity metrics (Fig. 2.4). It should be noted that echo width metrics did not show considerable superiorities compared to height related metrics under leaf-off condition. The most plausible explanation for this is that the difference of echo width metrics between coniferous species and deciduous species becomes narrower due to leaf loss of deciduous trees and the exposure of their branches, which, at the same time, can be well represented by geometric features. Those results indicated radiometric features have greater potential for representing species-specific characteristic, which is supported by previous studies (Heinzel and Koch 2011; Hovi et al. 2016; Kim et al. 2009; Ørka et al. 2009; Ørka et al. 2010; Sumnall et al. 2016). By contrast, many geometric features are affected by tree height, which may be also related to other properties, such as crown volume, crown shape and the interior structure of the tree crown. Misclassifications may be due to the similarities of the morphology among different tree species and also the architectural variations within the same tree species. The cause of structural deviations including tree competition as well as differences in environmental niche (such as temperature 
or soil condition) may also alter the shapes of tree crowns, which introduces further uncertainties in the classification. Therefore, discriminating tree species based on LiDAR derived geometric features may be challenging in complex natural forests, while using radiometric metrics provides better classification performance by their species-specific characters.

Several studies have discussed the influence of canopy conditions on tree properties from airborne LiDAR data (Kim 2007; Kim et al. 2009; Naesset 2005; Ørka et al. 2010). Our results showed that there was a slight improvement of classification accuracy when using leaf-off acquisition compared to leaf-on acquisition, which also has been supported by Reitberger et al. (2008) and Heurich (2006) in the same study area. However, there was no statistically significant difference in tree species mapping accuracy between the use of leafon and leaf-off LiDAR metrics (Table 2.6). We also found that a large proportion of important metrics were generated under leaf-off condition. It may be because the metrics derived under leaf-off condition offer a higher potential for representing the inner architecture of crowns, which give radiometric features a better chance to describe the interior structure of trees. Combining leaf-on and leaf-off datasets resulted in higher separability between tree species than using LiDAR data from one season. Notably, there was always a significant improvement in classification performance when combining leaf-on and leaf-off datasets compared to leaf-on dataset (Table 2.6). This result indicated that individual tree species discrimination could benefit more from the leaf-off acquisitions by obtaining more accurate species-specific LiDAR metrics. We gained an overall accuracy (OA) (66.5\%) and kappa value (0.58) for all six species when combining leaf-on and leaf-off datasets in site $\mathrm{B}$, which is slightly lower than many previous studies. However, we found a reasonable degree of accuracy by using solely airborne LiDAR data for individual tree species mapping in a natural mixed forest.

Ørka et al. (2010) indicated that there may be a set of features that can be applicable in tree species classification across different acquisitions. In this study, we tested and verified the robustness and transferability of important LiDAR metrics, which serve as the substantial markers of tree species that can be used for further study. It should be noted that our analysis was based on two study sites with relatively low variability regarding forest type and topography. Therefore, the applicability of the important metrics needs to be further tested in other study sites. Moreover, the point density of LiDAR data in this study is higher than in many other studies and the effect of point density on feature derivation and the 
classification still needs to be evaluated. Previous studies also indicated that high point density (i.e. $>40 \mathrm{pts} / \mathrm{m}^{2}$ ) airborne LiDAR data can be used to extract more detailed structural features such as internal foliage and branch patterns of an individual tree, which may further the distinction between different tree species (Li et al. 2013; Vauhkonen et al. 2014). Obtaining larger sample trees and improving individual tree segmentation accuracy may also lead to higher classification accuracy and better understanding of LiDAR metrics performance during classification.

\subsection{Conclusions}

This study has evaluated the performance of commonly used LiDAR metrics for discriminating tree species under leaf-on and leaf-off conditions. The results showed the radiometric metrics constantly contribute a higher accuracy compared to geometric features for tree species classification in a natural forest. The mean intensity of first-or-single returns was identified as the most robust LiDAR metrics which implied the major structural differences among tree species occur at the top crown layers and can be reflected in LiDAR metrics. Building links between the inherent architectural differences of tree species and how they manifest in LiDAR metrics remains a critical endeavour for remote sensing researchers. As a result, gathering field measurements and discovering relationships between crown structural characteristics and waveform metrics remains important to improve the physical interpretability of the airborne LiDAR metrics, with some of the relationships found here, serving as a ground for potential further investigations.

The accuracy of tree species classification using solely airborne LiDAR data is merely moderate. In the next chapter, we explore additional useful features derived from complementary remotely sensed data which further improve LiDAR-based tree species mapping. 


\section{Chapter 3}

\section{Improving LiDAR-based tree species mapping using multi-temporal CIR orthophotos *}

\footnotetext{
* This chapter is based on: Shi, Y., Wang, T., Skidmore, A.K., \& Heurich, M. (2020). Improving LiDAR-based tree species mapping in Central European mixed forests using multi-temporal digital aerial colour-infrared photographs. International Journal of Applied Earth Observation and Geoinformation, 84, 101970
} 


\begin{abstract}
Digital colour-infrared (CIR) aerial photographs, which have been collected routinely in many parts of the world over many decades, are an invaluable data archive for the monitoring and assessment of forest resources. Yet, the potential of these data for automated individual tree species mapping remains largely unexplored. One way to maximize the usefulness of digital CIR aerial photographs for individual tree species mapping is to integrate them with complementary remote sensing technologies such as the light detection and ranging (LiDAR) system and 3D segmentation algorithms. In this study, we examined whether multi-temporal digital CIR orthophotos could be used to further increase the accuracy of airborne LiDAR-based individual tree species mapping for a temperate mixed forest in eastern Germany. Our results showed that the texture features captured by multi-temporal digital CIR orthophotos under different view-illumination conditions were species-specific. As a consequence, combining these texture features with LiDAR metrics significantly improved tree species mapping accuracy (overall accuracy: 77.4\%, kappa: 0.68) when compared to using LiDAR data alone (overall accuracy: 69.3\%, kappa: 0.58). Among various texture features, the average gray-level in the near-infrared band was found to contribute most to the classification. Our results suggest that the synergic use of multi-temporal digital aerial photographs and airborne LiDAR data has the potential to accurately classify individual tree species in Central European mixed forests.
\end{abstract}




\subsection{Introduction}

Information about tree species are important inputs for biodiversity modelling and biomass estimation, and are indispensable for environmental, monitoring and protection activities (Waser et al. 2011). Remote sensing-assisted classification of individual tree species can aid forest management and ecosystem modelling and lead to more comprehensive and accurate forest inventories (Yin and Wang 2016). Over the last four decades, advances in remote sensing have enabled the classification of tree species using various sensor types (e.g. LiDAR, very high resolution digital aerial photographs, multispectral and hyperspectral sensors) (Fassnacht et al. 2016).

Aerial photographs have been acquired ever since the means have existed to lift cameras above the Earth's surface, beginning in the mid-19th century (Aber et al. 2010; Loetsch and Haller 1964). Historically, visual interpretation of aerial photographs was the most popular form of remote sensing for mapping trees in forests (Barrett et al. 2016; Spurr 1960). Captured at various spatial scales, aerial photographs are used for a wide range of purposes in resource management, from detailed surveys of individual trees to general land cover mapping over broad extents (Morgan et al. 2017). Traditional film-based aerial photographs are referred to as analogue images which have been routinely scanned and converted into digital images after the first decade of the 21 st century (Aber et al. 2010). With the technical advances in electronic devices and desktop computing, filmbased aerial photographs are rapidly becoming obsolete, due to the use of digital aerial photographs with increasingly sophisticated analysis methods. Specifically, small-format digital aerial photographs (equivalent to 35- and 70-mm film cameras) have established a niche that bridges the gap in scale and resolution between ground observations and imagery acquired from airborne and satellite sensors (Aber et al. 2010).

In recent decades, very high resolution digital colour-infrared (CIR) aerial photographs have been used to obtain information on individual tree species (Deng et al. 2016; Singh et al. 2015; Zhang and Hu 2012). Colour-infrared (CIR) aerial photography has long been recognized for its capabilities in land use, land cover and vegetation studies, including large-area and regional analysis, species differentiation and stress detection (Colwell 1960). Furthermore, digital CIR orthophotos obtained by georeferencing digital CIR aerial photographs using ground control points as well as GPS/INS system provide the true orthographic positions, which can be used for making direct measurements of distances, angles, 
directions, positions and areas without corrections for distortions. Manual (visual) interpretation of aerial photography has long been a standard procedure in developing forest inventories; however, human interpretations can be subjective, inconsistent, and labour-intensive (Koch et al. 2002). Although very high resolution digital CIR orthophotos allow specialists to recognize patterns of species, visual interpretation may not always fully reveal the spectral and texture information of individual trees. The variability among the same tree species and the similarity between different tree species present additional challenges (Dechesne et al. 2017).

Previous research has shown that there are species-specific differences in how the observed brightness changes when the viewing direction in digital aerial photography is altered (Korpela et al. 2014). Studies also pointed out that the spectral separability of tree species is partly dependent on the view-illumination geometry and the reflectance anisotropy, and the high within-species reflectance variation in digital aerial photographs may affect the spectral signatures of trees (Dechesne et al. 2017; Korpela et al. 2011; Leckie et al. 2005). The collection of very high resolution digital aerial photographs has become a routine forest inventory procedure in many countries. This provides an opportunity to capture brightness variation patterns within tree crown by using multi-temporal information. Korpela et al. (2011) concluded that "the brightness variation inside crowns showed band- and species- specific differences" and "the reflectance differences observed in the various bands and geometries could potentially be taken into account for the improvement of species classification". However, for individual tree species classification, the capability of texture information derived from multi-temporal digital aerial photographs under different illumination conditions remains largely unexplored.

Recent advances in remote sensing technology and machine learning methods provide avenues to further explore the potential of digital CIR orthophotos for individual tree species mapping. Processing capabilities allow us to efficiently generate spectral and texture features from digital CIR orthophotos, while machine learning algorithms offer a means to combine these features with complementary data sources. For instance, LiDAR data provide a range of metrics related to both the geometric and radiometric characteristics of trees. While geometric features describe the architecture of tree crowns and branching patterns, radiometric features are more related to foliage material, leaf orientation and density (Kim et al. 2009). In addition, directional reflectance signatures and crown texture information derived from digital aerial photographs can be used 
for forest monitoring and species classification (Korpela et al. 2014; Kuzmin et al. 2016; Singh et al. 2015; Tian et al. 2017). Crown texture often reflects the spatial variation of crown-internal shadows, foliage properties (size, density, reflectivity) and branching patterns (Sayn-Wittgenstein 1978). On small format CIR orthophotos, leaves, branches and inner tree crown shadows all contribute to the texture information (Haara and Haarala 2002). New perspectives for regional and national mapping approaches are emerging through digital aerial photographs, which are likely - in contrast to airborne LiDAR data - to be updated more regularly by regional or national mapping agencies (Waser et al. 2017). Although the combination of airborne LiDAR data and texture features derived from individual digital aerial photographs has been successfully employed for tree species monitoring (Kulikova et al. 2007) and land cover mapping (Silveyra Gonzalez et al. 2018), the utility of texture information derived from multitemporal digital CIR orthophotos in aiding LiDAR-based tree species mapping has not been tested.

The current study aims to evaluate the role of multi-temporal digital CIR orthophotos in improving LiDAR-based individual tree species classification in Central European mixed forests. Specifically, we set out to (1) examine the correlation of texture features derived from multi-temporal digital CIR orthophotos; (2) integrate texture information derived from multi-temporal digital CIR orthophotos with airborne LiDAR derived metrics for tree species classification; and (3) assess the utility of combining multi-temporal digital CIR orthophotos and LiDAR data for tree species discrimination and identify the most valuable features from both datasets.

\subsection{Materials and Methods}

\subsubsection{Study sites and field data}

The Bavarian Forest National Park (BFNP) was the first National Park established in Germany, in 1970, and is situated in south-eastern Germany along the border with the Czech Republic. As a mixed temperate forest reserve in Central Europe, it covers 24,218 hectares with elevations between 600 and 1453 $\mathrm{m}$ above sea level (Cailleret et al. 2014). Within the park, three major forest types exist: above $1100 \mathrm{~m}$ there are sub-alpine spruce forests of Norway spruce (Picea abies) and some Mountain ash (Sorbus aucuparia) (high altitude); on the slopes, between 600 and $1100 \mathrm{~m}$, are mountains of mixed forests of Norway spruce, Silver fir (Abies alba), European beech (Fagus sylvatica), and sycamore maple 
(Acer pseudoplatanus); in wet depressions that often experience cold air pockets in the valley bottoms, spruce forests of Norway spruce, Mountain ash, and white birches (Betula pendula) occur (valley bottoms) (Heurich 2008).

Two study sites (approximately 25 hectares for each site) were selected based on the species richness in the BFNP (Fig. 3.1). The field data was collected in July 2016 and July 2017, including 256 locations of individual tree species at site A and 193 locations of individual tree species at site B. A Leica Viva GS10 Plus differential GPS (Leica Geosystems AG, Heerbrugg, Switzerland) was used to record the spatial location of trees in the field. After post-processing, the absolute error of the differentially corrected coordinates was within $0.25 \mathrm{~m}$ (see Chapter 2).

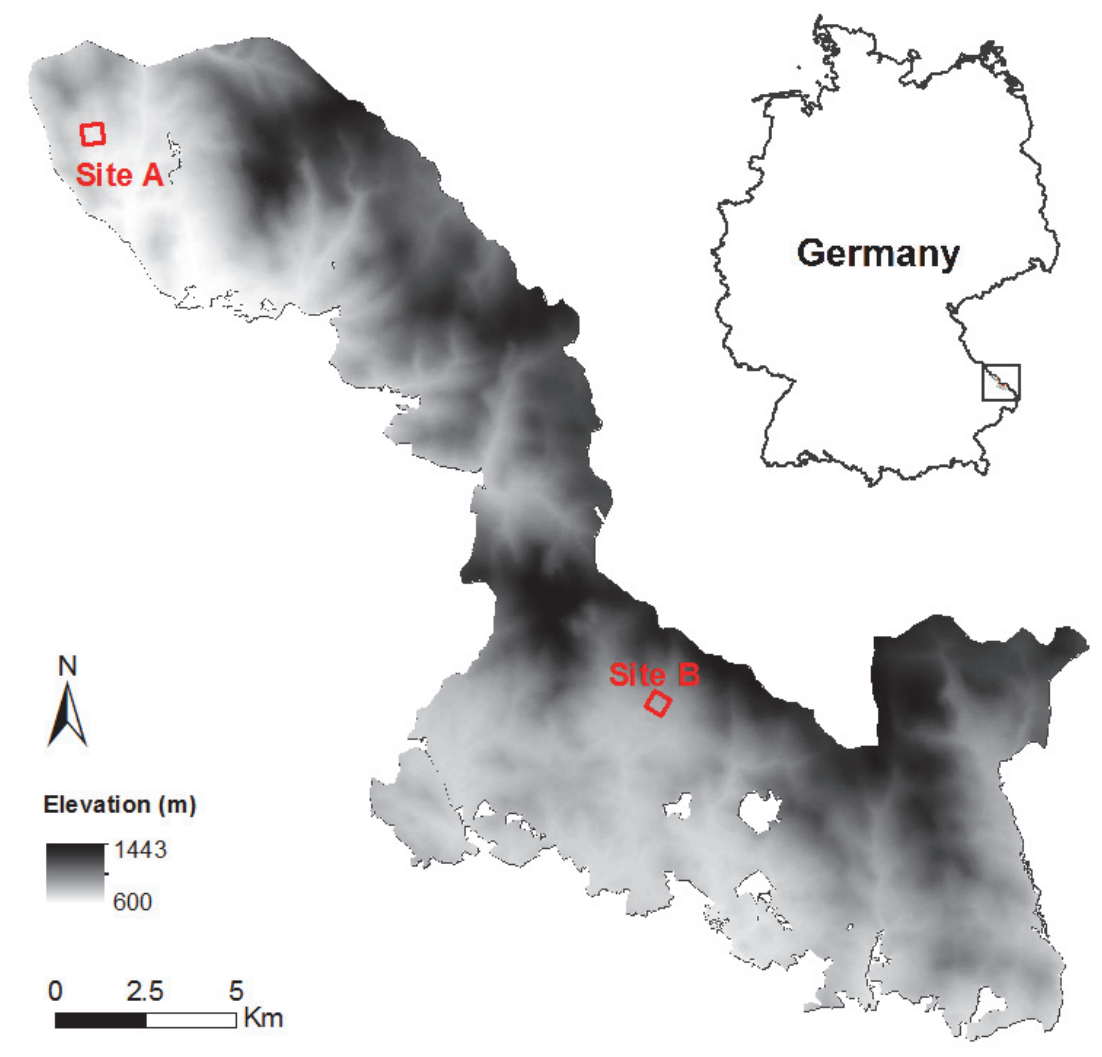

Fig. 3.1 Location of the two study sites in Bavaria Forest National Park, Germany 


\subsubsection{Multi-temporal digital CIR orthophotos}

Three digital CIR orthophotos were utilised in this study, from 6 June 2015, 23 June 2016 and 11 June 2017, covering the whole BFNP. All three digital CIR images were recorded using Intergraph's Digital Mapping Cameras (DMC) from an altitude of approximately $2900 \mathrm{~m}$. Four parallel multispectral colour DMCs were utilised to form composite images, and each of them had a resolution of $3456 \times 1920$ pixels with a virtual pixel size of $12 \mu \mathrm{m}$. The nominal focal length of the cameras was $12 \mathrm{~mm}$. The digital CIR images consist of three bands: NIR (near-infrared) $(675-850 \mathrm{~nm})$, Red $(590-675 \mathrm{~nm})$ and Green $(500-650 \mathrm{~nm})$. Finally, all three digital CIR images were radiometrically corrected and orthorectified by using optimal camera calibration observations, transformation parameters and ground control points. The procedures were conducted in the program system OrthoBox (Orthovista, Orthomaster) from the Trimble/INPHO. The detailed information about spatial resolution, average flying altitude, flight time and sun position of three digital CIR orthophotos are shown in Table 3.1.

Table 3.1 Detailed information of multi-temporal digital CIR orthophotos used in this study.

\begin{tabular}{llll}
\hline $\begin{array}{l}\text { Digital CIR } \\
\text { orthophotos }\end{array}$ & 6 June 2015 & 23 June 2016 & 11 June 2017 \\
\hline Spatial resolution & $20 \mathrm{~cm}$ & $20 \mathrm{~cm}$ & $10 \mathrm{~cm}$ \\
Average altitude & $2909 \mathrm{~m}$ & $2918 \mathrm{~m}$ & $2879 \mathrm{~m}$ \\
Flight time & $09: 50-12: 05$ & $09: 50-12: 15$ & $10: 30-13: 25$ \\
Sun position & $44^{\circ}-57^{\circ}$ & $43^{\circ}-62^{\circ}-53^{\circ}$ & $49^{\circ}-64^{\circ}-35^{\circ}$ \\
\hline
\end{tabular}

\subsubsection{Airborne LiDAR data}

An airborne LiDAR flight campaign was conducted out on 18 August 2016, using a Riegl LMS-Q680i scanner integrated in a full-waveform laser scanning system. The system operated at a wavelength of $1550 \mathrm{~nm}$ and was flown at approximately $300 \mathrm{~m}$ above terrain elevation. The pulse repetition frequency was $400 \mathrm{kHz}$ with a maximum scanning angle of $\pm 15^{\circ}$. The average point density was about 70 $\mathrm{pts} / \mathrm{m}^{2}$. Four transects in the park were covered by 21 flight lines with $30 \%-50 \%$ strip overlaps. The preparation of the LiDAR data was described in Chapter 2, section 2.2.2. 


\subsubsection{Individual tree delineation}

The automatic delineation of individual tree crowns was performed using an enhanced approach, which detects individual trees in multi-layered forests with an integrated 3D segmentation proposed by Yao et al. (2013). This method is based on 3D segmentation combining mean shifts with normalized cuts, which can exploit the advantages of full waveform data and detect supressed trees in the both middle and lower layers. Yao et al. (2013) achieved a detection rate of up to $70 \%$ for trees in the upper layer and found that tree detection rates using this method improved by $10 \%$ to $25 \%$ for all three forest layers compared to that obtained by the method presented in Reitberger et al. (2009) and Yao et al. (2012).

Field measured trees that were discernible both in the LiDAR segments and in the multi-temporal digital CIR orthophotos formed the potential reference trees for this study (Fig. 3.2). To link the field measured trees to both LiDAR and digital CIR orthophotos, we firstly overlaid the post-processed DGPS tree locations with tree crown segments and georeferenced digital CIR orthophotos; and secondly we identified the field measured trees which located within the crown segments and visible in digital CIR orthophotos. Finally, a visual verification was carried out with the assistance of the photos of the measured trees and the species of neighbouring trees recorded during the fieldwork. To reduce linking errors, trees undetected by the segmentation or assigned to more than one segment were removed from further analysis. In the end, 58 beech trees, 40 birch trees, 60 fir trees, 55 maple trees and 57 spruce trees ( 270 sample trees in total) from two study sites were selected for further analysis. 

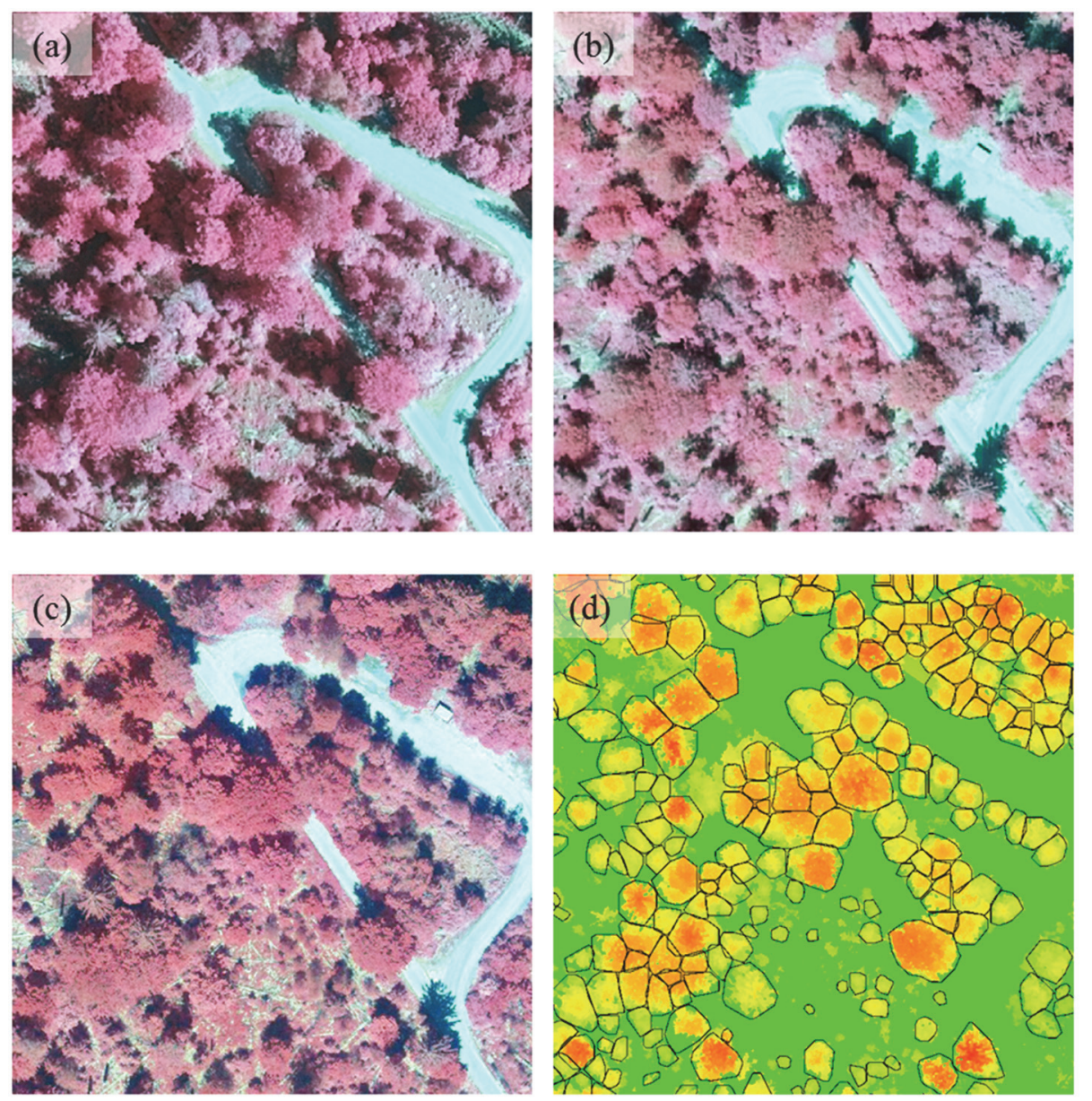

Fig. 3.2 The digital CIR orthophotos of a sample area from 2015 (a), 2016 (b) and 2017 (c). The 3D segmentation of tree crown is displayed with the background of the LiDARderived canopy height model (d).

\subsubsection{Feature derivation}

\subsubsection{Geometric and radiometric features from LiDAR data}

Once the sample trees were correctly linked to the 3D segments, the normalized point clouds within the segments were extracted to derive LiDAR metrics for each sample tree. The derived LiDAR metrics can be grouped into four categories: (1) height distribution (including maximum, mean, standard deviation, coefficient of variation, skewness, kurtosis, variance, 25th percentile and 95th percentile of the normalized height); (2) crown shape (including the ratio of crown base height to tree height, the ratio of crown volume to crown area, and 
canopy relief ratio); (3) point distribution (including the percentage of first returns to all returns, the percentage of last returns to all returns, first returns above mean height, and all returns above $2 \mathrm{~m}$ ); and (4) intensity and echo width metrics (including the abovementioned statistical variables applied for height distribution, the mean value of the first-or-single returns, and the mean value of single returns). As a result, 40 LiDAR metrics were assigned to each sample tree. A detailed description of the generated LiDAR metrics can be found in Table 2.3.

\subsubsection{Texture features from multi-temporal digital CIR orthophotos}

The Grey Level Co-Occurrence Matrix (GLCM) is one of the best known texture analysis methods (Haralick et al. 1973). The texture features obtained by the GLCM method have been widely employed in the remote sensing community for forest modelling (Kayitakire et al. 2006; Ozdemir et al. 2008; Pasher and King 2010) and tree species classification (Kuzmin et al. 2016; Singh et al. 2015). Based on the crown polygons delineated by 3D segmentation, we used the GLCM to derive texture features of each sample tree. Texture features were calculated for each pixel within a tree crown, based on a window size of 3 by 3 pixels (i.e. each pixel and its 8 neighbours). We used the following eight texture parameters: mean, homogeneity, contrast, dissimilarity, entropy, variance, angular second moment and correlation. After calculating the parameters for all the pixels within an individual tree crown, we used the mean value of each parameter to represent the texture features of each sample tree. All texture features were normalized for clarity. In total, 72 texture features, generated from the NIR, red and green bands from three years of digital CIR orthophotos, were assigned to each sample tree. The description of the texture features is shown in Table 3.2. The derivation of texture features was performed in the R language environment (http://www.rproject.org/). 
Table 3.2 Description of generated texture features. In formulas, $i$ and $j$ are row and column numbers, respectively. $N$ is the total number of pixels. $u_{i}, u_{j}, \sigma_{i}{ }^{2}$, and $\sigma_{j}{ }^{2}$ are the means and standard deviations of $P_{i}$ and $P_{j} . P(i, j)$ is the normalized co-occurrence matrix.

\begin{tabular}{|c|c|c|}
\hline GLCM texture features & Formula & Description \\
\hline MEAN & ${ }_{j=0}^{\mathrm{v}-1} i P_{i, j}$ & $\begin{array}{l}\text { The average gray level in the } \\
\text { local window }\end{array}$ \\
\hline Homogeneity (HOM) & $i \frac{P_{i j}}{1+(i-j)^{2}}$ & $\begin{array}{l}\text { A measure of lack of variability } \\
\text { or the amount of local of } \\
\text { similarity in an image }\end{array}$ \\
\hline Contrast (CON) & ${ }_{i, j=0}^{N-1} i P_{i j}(i-j)^{2}$ & $\begin{array}{l}\text { A measure of the amount of local } \\
\text { variation in pixels values among } \\
\text { neighbouring pixels }\end{array}$ \\
\hline Dissimilarity (DIS) & ${ }_{j=0}^{-1} i P_{i, j}|i-j|$ & $\begin{array}{l}\text { A measure of lack of similarity } \\
\text { in an image }\end{array}$ \\
\hline Entropy (ENT) & $i P_{i, j}\left(-\ln P_{i, j}\right)$ & $\begin{array}{l}\text { A measure of the degree of } \\
\text { disorder in an image }\end{array}$ \\
\hline Variance (VAR) & $\sum_{i, j=0}^{N-1} P_{i j}\left(i-u_{i}\right)^{2}$ & $\begin{array}{l}\text { A measure of the degree of } \\
\text { variation in an image }\end{array}$ \\
\hline $\begin{array}{l}\text { Angular second } \\
\text { moment (ASM) }\end{array}$ & $\sum_{i, j=0}^{N-1} i P_{i, j}^{2}$ & $\begin{array}{l}\text { A measure of textural uniformity } \\
\text { or pixel pair repetitions }\end{array}$ \\
\hline Correlation (COR) & $\sum_{i, j=0}^{N-1} P_{i, j}\left(\frac{\left(i-u_{i}\right)\left(j-u_{j}\right)}{\sigma_{i} \sigma_{j}}\right)$ & $\begin{array}{l}\text { A measure of gray level linear } \\
\text { dependencies in an image }\end{array}$ \\
\hline
\end{tabular}

\subsubsection{Feature selection and tree species classification}

Many of the second-order texture measures proposed by Haralick et al. (1973) have been found to be highly correlated (Clausi 2002). The selection of the most relevant texture features should be therefore implemented before classification (Rodriguez-Galiano et al. 2012). As such, we examined the correlation coefficient, and its significance, between each pair of texture features. The test of the significance of the correlation coefficient was performed to assess whether the linear relationship between the input features was strong enough to consider elimination. We ranked the texture features by the frequency of low correlation $(|r|<0.70)$ and high insignificance $(p>0.05)$ with other features, and the top 10 texture features of each year were used for further analysis, along with selected LiDAR metrics. The selection of LiDAR metrics was based on Chapter 2.

The Random Forest algorithm was used for feature selection and classification (see chapter 2, section 2.2.6 for a detailed description). In total, 22 variables were selected for tree species classification, including 12 LiDAR metrics and 10 texture features derived from the multi-temporal digital CIR orthophotos. Two tuning parameters are required in the Random Forest classifier: the number of trees "Ntree" and the number of predictors sampled for splitting at each node 
"Mtry". To obtain the optimal combination of the parameters for best classification performance, we tested Random Forest models by varying "Ntree" from 1 to 500 and "Mtry" from 1 to 30. The resulting models were evaluated using the kappa index (Cohen 1960). The model with the highest kappa coefficient was chosen as the best model.

Accuracy of the models were assessed using overall accuracy, producer's and user's accuracy, and the kappa coefficient. We also employed McNemar's test (McNemar 1947) to determine if statistical significant differences occur among classifications using different datasets. The feature selection and classification procedures were carried out using the R package "randomForest" (Liaw and Wiener 2002).

\subsection{Results}

\subsubsection{Correlation and feature selection}

High correlations $(|r|>0.7)$ were observed between the texture features of the NIR, red and green bands generated from the digital CIR orthophotos of the same year (Fig. 3.3a). The average gray level (MEAN) showed the lowest correlations with other texture features between the three years. When comparing the texture features between different years, the angular second moment (ASM), homogeneity (HOM) and entropy (ENT) features all showed high correlations with each other. When comparing the texture features from the same year, high correlation occurred between the angular second moment (ASM), homogeneity $(\mathrm{HOM})$, contrast $(\mathrm{CON})$ and variance (VAR) features.

Fig. 3.3b shows the correlation and significance between texture features from 2015. The features were ranked by the frequency of low correlation $(|r|<0.70)$ and high insignificance $(p>0.05)$ with other features. As a result, the top 10 features were selected from 2015: DIS_NIR, VAR_NIR, VAR_G, CON_NIR, ASM_NIR, ASM_R, ASM_G, MEAN_NIR, MEAN_R, and MEAN_G (See Table 3.2 for the definitions of the texture features. R, G, and NIR represent the texture features that were derived from the red, green and near-infrared bands, respectively). Similarly, the 10 top-ranked texture features were selected from the years 2016 and 2017.

Table 3.3 lists the selected variables derived from airborne LiDAR and multitemporal digital CIR orthophotos for the classification. 9 out of 12 selected LiDAR metrics were radiometric metrics (intensity and echo width related 
metrics). Among 10 selected texture features, 7 were generated from NIR bands. The average gray level (MEAN) and the dissimilarity (DIS) were the most frequently selected texture features.

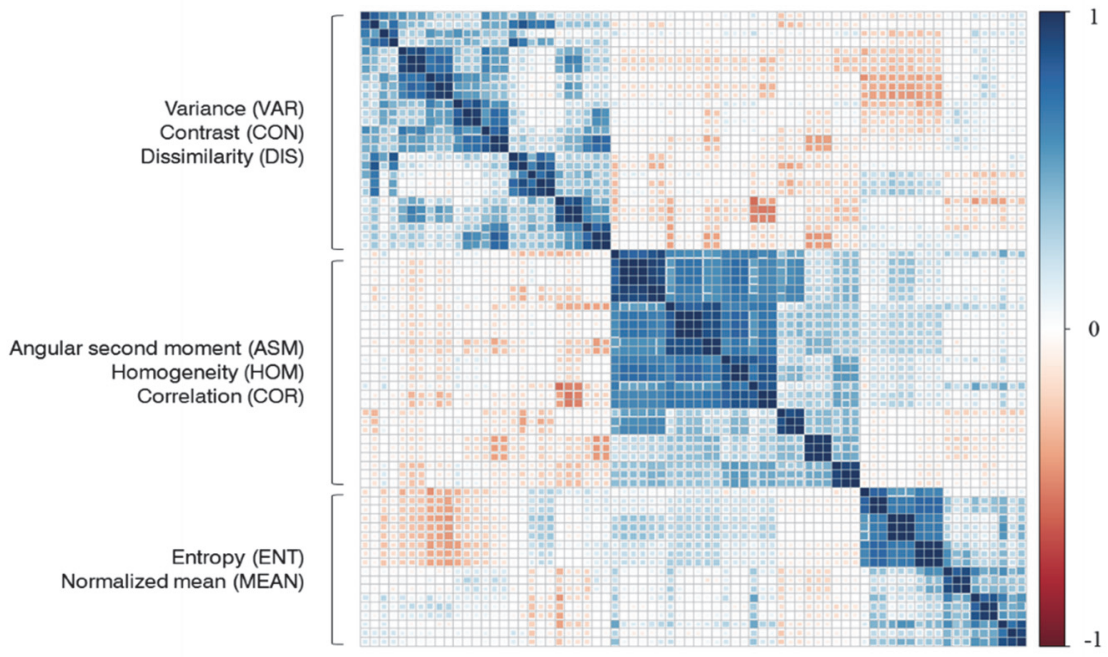

(a)

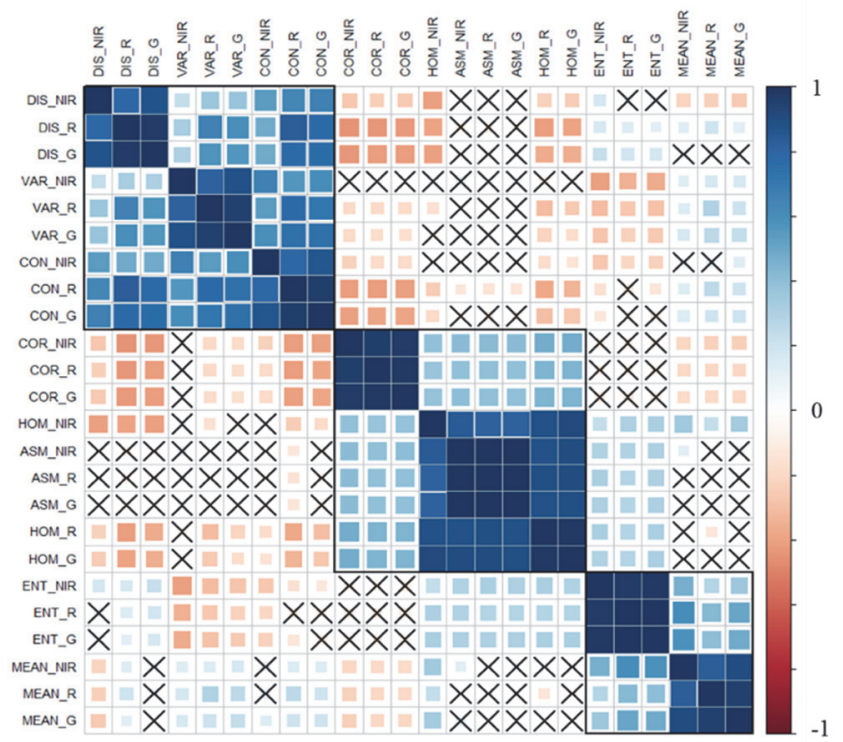

(b)

Fig. 3.3 The cross-correlation matrix of the texture features derived from multi-temporal digital CIR orthophotos (a) and the combined correlogram with the significance test of 2015 (b). Blue colours indicate positive correlations and red colours indicate negative correlations. The insignificant values $(p>0.05)$ were marked with black crosses. The features were sorted by hierarchical clustering order (black rectangles). 
Table 3.3 Selected variables derived from LiDAR and digital CIR orthophotos for classification

\begin{tabular}{ll}
\hline Input variables & Index or description \\
\hline LiDAR metrics (12) & \\
Imean_first & The mean intensity of first-or-only returns \\
Ip75 & The 75th percentile of intensity \\
Height & The height of tree \\
Ewmean_first & The mean echo width of first-or-single returns \\
EWmean & The mean value of echo width \\
Icv & The coefficient variation of intensity \\
Ewmean_single & The mean echo width of single returns \\
Imean_single & The mean intensity of single returns \\
Imean & The mean intensity \\
Ivar & The variation of intensity \\
Hvar & The variation of height \\
Hsd & The standard deviation of height \\
Texture features (10) & \\
2015_MEAN_NIR & The average gray level of NIR band from 2015 \\
2016_MEAN_NIR & The average gray level of NIR band from 2016 \\
2017_DIS_NIR & The dissimilarity of NIR band from 2017 \\
2015_MEAN_G & The average gray level of green band from 2015 \\
2015_DIS_NIR & The dissimilarity of NIR band from 2015 \\
2017_MEAN_NIR & The average gray level of NIR band from 2017 \\
2016_DIS_R & The dissimilarity of red band from 2016 \\
2015_ASM_NIR & The angular second moment of NIR band from 2015 \\
2016_DIS_G & The dissimilarity of green band from 2016 \\
2017_VAR_NIR & The variance of NIR band from 2017 \\
\hline
\end{tabular}

\subsubsection{Classification performance from different combinations of datasets}

Table 3.4 shows the confusion matrices and corresponding producer's and user's accuracies, the overall accuracy and kappa coefficient for classification results using different feature combinations. The best classification accuracy was achieved with a model containing both LiDAR metrics and texture variables from three years of digital CIR orthophotos: overall accuracy of $77.4 \%$ with a 0.68 kappa coefficient. The classifications using only LiDAR and only CIR texture features yielded similar results, with overall accuracies of $69.3 \%$ and $66.7 \%$, 
respectively. Birch trees had the highest user's accuracy in each of the models. The lowest user's and producer's accuracies occurred for the fir and maple trees using only multi-temporal texture features. The highest misclassifications were found between the pairs of beech and maple, and fir and spruce.

Table 3.5 shows the significance levels between classification results generated from different combinations, based on $p$ values in the McNemar's test. Combining LiDAR and texture features from three years of digital CIR orthophotos significantly improved the accuracy compared to using LiDAR and digital CIR orthophotos alone. However, the combination of one or two years of digital CIR orthophotos with LiDAR data did not yield statistically significant improvements compared to using LiDAR data alone. Moreover, there was no statistically significant difference between the classification performance of using only LiDAR or digital CIR orthophotos. 


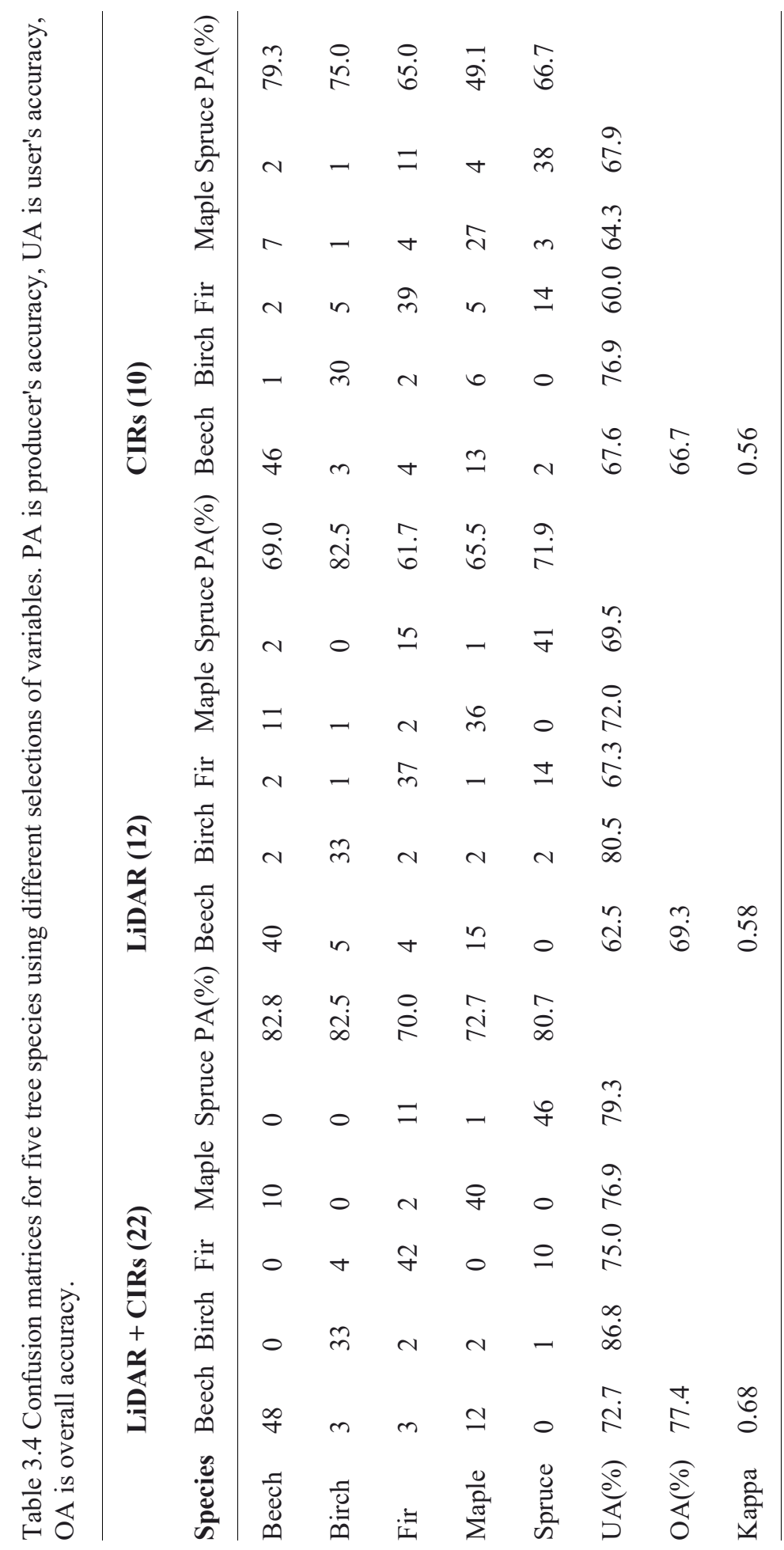


Table 3.5 McNemar's test for pairwise comparison between classification results using different combinations. CIR $(\times 3)$ means all three years of digital CIR orthophotos; CIR $(\times 2)$ means any two years of digital CIR orthophotos; CIR $(\times 1)$ means any one year of digital CIR orthophoto. ${ }^{* *}: p<0.001 ; * *: p<0.01 ; *: p<0.05 ; \mathrm{NS}: p>0.05$.

\begin{tabular}{|c|c|c|c|c|c|}
\hline & $\begin{array}{l}\text { LiDAR+ } \\
\text { CIR }(\times 3)\end{array}$ & $\begin{array}{l}\text { LiDAR }+ \\
\text { CIR }(\times 2)\end{array}$ & $\begin{array}{l}\text { LiDAR+ } \\
\text { CIR }(\times 1)\end{array}$ & LiDAR & $\begin{array}{l}\text { CIR } \\
(\times 3) \\
\end{array}$ \\
\hline $\begin{array}{l}\text { LiDAR+ } \\
\text { CIR }(\times 3)\end{array}$ & - & $* *$ & $* * *$ & $* * *$ & $* * *$ \\
\hline $\begin{array}{l}\text { LiDAR+ } \\
\text { CIR }(\times 2)\end{array}$ & & - & NS & NS & $*$ \\
\hline $\begin{array}{l}\text { LiDAR+ } \\
\text { CIR }(\times 1)\end{array}$ & & & - & NS & NS \\
\hline LiDAR & & & & - & NS \\
\hline $\operatorname{CIR}(\times 3)$ & & & & & - \\
\hline
\end{tabular}

\subsubsection{The contribution of selected features for tree species discrimination}

Fig. 3.4 shows the relative importance of the selected variables for the classification based on the MDA index. In the combined model (LiDAR metrics and texture features), the three most important variables were LiDAR metrics (Imean_first, Ip75 and Height), followed by the average gray level of the NIR bands from 2015 and 2016. Specifically, the top-ranked LiDAR metric was the mean intensity of first-or-single returns (Imean_first) and the top-ranked texture feature was the average gray level of the NIR band from 2015 (2015_MEAN_NIR). The average gray level and the dissimilarity of NIR bands were found to be more important than other texture features (e.g. contract, variance and the angular second moment).

Table 3.6 shows five top-ranked features which contributed most to the discrimination of one tree species from the others in the classification. The texture features derived from the NIR bands of the multi-temporal digital CIR orthophotos contributed most to the discrimination of beech and spruce from the other species. On the contrary, birch and maple were most distinguishable by using LiDAR metrics - echo width and height related metrics contributed most for the classification of birch, while intensity related metrics were more important for the identification of maple. 
Fig. 3.5 shows the inter-species comparison of different variables derived from multi-temporal digital CIR orthophotos and LiDAR data. The average gray level and the dissimilarity of NIR bands (MEAN_NIR and DIS_NIR) from different years have similar patterns, however, the differences between species vary between years. For example, the difference of the average gray level of the NIR band (MEAN_NIR) between beech and birch was more pronounced in 2015 (first row in Fig. 3.5), while the difference in the dissimilarity of the NIR band (DIS_NIR) became more distinguishable between maple and spruce in 2016 (second row in Fig. 3.5). Out of the most important LiDAR metrics (e.g. Imean_first, Ewmean and Height), the mean intensity of first-or-single returns (Imean_first) distinguished beech and maple from other species. In contrast, the mean value of echo width (Ewmean) was the most important feature for the discrimination of birch (Table 3.6).

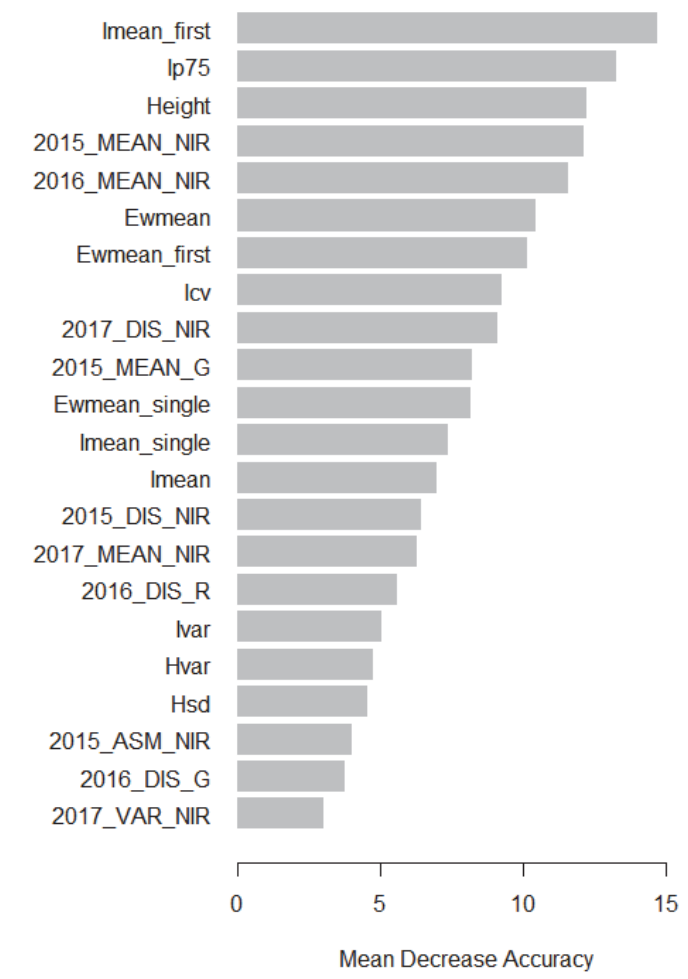

Fig. 3.4 The relative importance of the selected variables for tree species classification. 
Table 3.6 Top five most important variables for discriminating each tree species in the classification. See Table 3.3 for the definitions of the metrics.

\begin{tabular}{llllll}
\hline & Beech & Birch & Fir & Maple & Spruce \\
\hline 1 & 2015_MEAN_NIR & Ewmean & Imean_first & Ip75 & 2016_MEAN_NIR \\
2 & 2016_MEAN_NIR & Ewmean_first & Ip75 & Imean_first & 2015_DIS_NIR \\
3 & 2015_MEAN_G & Height & Imean_single & Icv & 2017_DIS_NIR \\
4 & 2017_DIS_NIR & Hvar & Height & Imean & Ewmean_single \\
5 & Imean_first & Hsd & 2015_MEAN_NIR & Ivar & Height \\
\hline
\end{tabular}
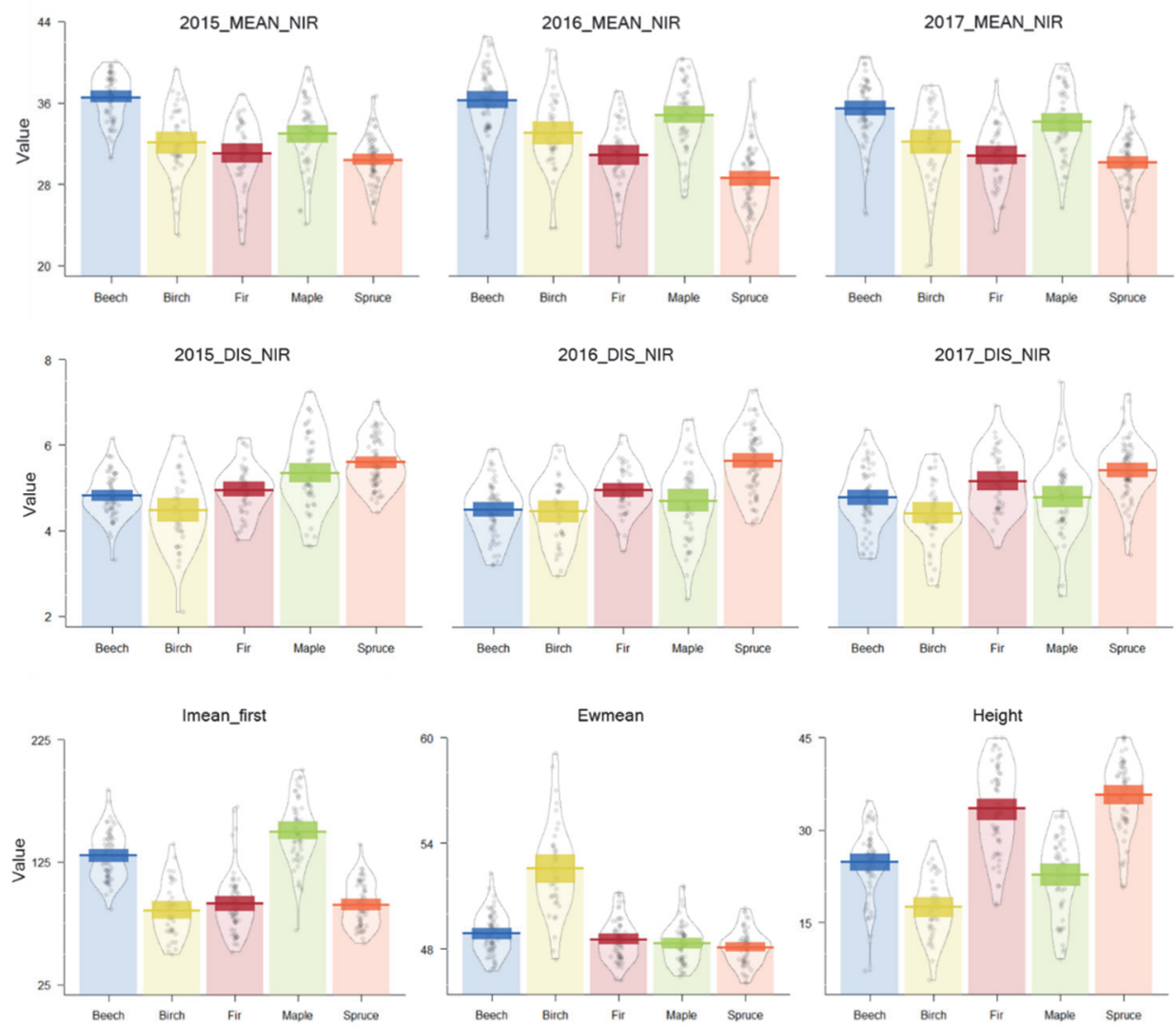

Fig. 3.5 Interspecies comparison of different variables derived from multi-temporal digital CIR orthophotos and LiDAR data. The first row is the average gray level of the NIR band (MEAN_NIR) from 2015, 2016 and 2017 digital CIR orthophotos; the second row is the dissimilarity of the NIR band (DIS_NIR) from 2015, 2016 and 2017 digital CIR orthophotos; and the last row is the mean intensity of first-or-only returns (Imean first), the mean value of echo width (Ewmean), and the tree height (Height) derived from airborne LiDAR data. 


\subsection{Discussion}

In this study, we explored the potential of using texture features derived from multi-temporal digital CIR orthophotos to improve LiDAR-based individual tree species classification in a temperate mixed forest in Germany. Our study found that adding texture features from multi-temporal digital CIR orthophotos to LiDAR-based tree species classification significantly increased classification accuracy. In particular, the average gray level and the dissimilarity of the NIR band within a tree crown, derived from multi-temporal digital CIR orthophotos, were found to provide valuable information for discriminating tree species. However, we did not find significant improvements between the results of using only one or two years of digital CIR orthophotos with LiDAR data and that of using LiDAR data alone. This finding aligns with Korpela et al (2014), who used a single year of aerial photography and concluded that interspecies differences in directional reflectance anisotropy do not constitute a significant improvement to tree species classification. However, our results showed that the texture features derived over three years from digital CIR orthophotos resulted in a significant improvement to the classification performance compared with only using LiDAR data. The texture features derived from the same year are more likely to be highly correlated than those from different years, which may partly explain why adding one year or two years of digital CIR orthophotos did not yield significant improvements. The spectral variance within species hampered the ability of using only digital CIR orthophotos for species identification, which highlights the benefits of fusing multiple sources of data.

While digital aerial photographs have been used as complementary data sources for LiDAR-based individual tree species mapping, they have been mainly used to visually identify tree tops, tree crowns and tree types (conifers or deciduous trees) (e.g. Korpela 2006; Koukoulas and Blackburn 2005; Persson et al. 2004). In our study, the tree crowns were delineated from high density airborne LiDAR data using a 3D segmentation algorithm. In comparison to manual delineation of tree crown from digital aerial photographs, which usually eliminates the shaded parts (e.g. Heinzel et al. 2008; Persson et al. 2004), LiDAR-based tree crown delineation provides an opportunity to automatically generate more complete tree crowns, without the influence of shadow effects. This allows species-specific information to be captured by the texture patterns within a tree crown, especially the texture features derived from multi-temporal digital aerial photographs with different illumination geometry. Although multi-temporal digital aerial photographs are not always able to capture seasonal changes, the variation of 
brightness patterns under different view-illumination conditions still provides valuable information for individual tree species classification. Our study demonstrates the potential of improving tree species mapping with the assistance of archived digital aerial photographs, which are widely available in many countries. It should be noted that our study was conducted in a temperate mixed forest in Central Europe with relatively low variability regarding forest type and tree species. Therefore, the potential capability of multi-temporal digital aerial photographs for LiDAR-based individual tree species classification in other forest ecosystems or species-rich habitats requires further investigation.

Our results highlighted the importance of texture features derived from the NIR band of digital aerial photographs in discriminating between tree species. The average gray level of the NIR band was found to be less correlated with other texture features and contributed more to the classification. This is most likely due to its sensitivity to illumination variation and foliage. Similar results were reported by Coops et al (2004), who found a greater discrimination between canopy and understory vegetation in the NIR band than the green band. In our study, beech and spruce showed distinct differences in the NIR band, and in fact the texture features of the NIR band contributed more than LiDAR metrics to the discrimination of beech and spruce from other species (Table 3.6). The dissimilarity of the NIR band was another texture feature that was identified as relatively important in tree species classification. In agreement with our previous study (Shi et al. 2018a), radiometric LiDAR metrics (intensity and echo width related metrics) played an important role in the tree species classification - by well representing the structural and morphological characteristics of the tree crown. Our results demonstrated that LiDAR metrics were more capable than texture features in distinguishing tree species. For example, using the mean value of echo width (Ewmean), we were able to distinguish birch from other four species.

Digital aerial photographs have several advantages over hyperspectral and LiDAR data, including lower deployment and data collection costs, easier access to a variety of platforms, and the opportunity for forest managers to capture and process data over a long time span. As reported by Key et al. (2001), phenological information such as spring leaf-out, leaf maturity, and autumn senescence captured by multi-temporal digital aerial photographs are important indicators in the spectral separation of individual tree species. Digital aerial photographs taken in the spring, shortly after the flushing of leaves, or in autumn, after the trees have senesced (Holmgren et al. 2008), would be able to provide more species-specific 
information for species identification. We note that system differences and misregistration between images may hamper the utility of multi-temporal datasets, especially those from different platforms and sensors. Furthermore, the spectral bands available in most commercial sensors are located in the blue, green, red and near-infrared parts of the spectrum, which only describe the differences between the tree species across limited wavelengths. As discussed by Heikkinen et al. (2010), tree species classification based on multispectral images could be improved by including the red edge region of the spectrum, which is more unique to tree species, age and health (Cho and Skidmore 2006). Future decisions regarding the use of multispectral sensors for mapping tree species at the individual tree level should be made with consideration of the trade-offs between spatial resolution, spectral resolution, flight season and revisit cycle.

\subsection{Conclusions}

To our knowledge, this is the first study that investigates how archived multitemporal digital aerial colour-infrared photographs can be used to improve LiDAR-based individual tree species mapping. Our results show that the texture features generated from multi-temporal digital CIR orthophotos under different view-illumination conditions are species-specific. Combining these texture features with LiDAR metrics was shown to significantly improve the individual tree species mapping accuracy in a temperate mixed forest in eastern Germany. Specifically, the average gray-level and the dissimilarity of the NIR band contributed most (among texture features) to the classification. Our results demonstrate that the fusion of multi-temporal digital aerial photographs with airborne LiDAR data can accurately classify individual tree species in Central European mixed forests.

The main restriction of digital aerial photographs for discriminating individual tree species is the imagery can only portray the differences between tree species across limited wavelengths. Hyperspectral imagery, on the contrary, provides hundreds of available wavebands for reflecting the species-specific traits. The next chapter combines hyperspectral and LiDAR data for species-specific traits retrieving and tree species classification. 


\section{Chapter 4}

\section{Tree species classification using remotely sensed plant functional traits *}

* This chapter is based on: Shi, Y., Skidmore, A.K., Wang, T., Holzwarth, S., Heiden, U., Pinnel, N., Zhu, X., \& Heurich, M. (2018). Tree species classification using plant functional traits from LiDAR and hyperspectral data. International Journal of Applied Earth Observation and Geoinformation, 73, 207-219 


\begin{abstract}
Plant functional traits have been extensively used to describe, rank and discriminate species according to their variability between species in classical plant taxonomy. However, the utility of plant functional traits for tree species classification from remote sensing data in natural forests has not been clearly established. In this study, we integrated three selected plant functional traits (i.e. equivalent water thickness $(\mathrm{Cw})$, leaf mass per area $(\mathrm{Cm})$ and leaf chlorophyll (Cab)) retrieved from hyperspectral data with hyperspectral derived spectral features and airborne LiDAR derived metrics for mapping five tree species in a natural forest in Germany. Our results showed that when plant functional traits were combined with spectral features and LiDAR metrics, an overall accuracy of $83.7 \%$ was obtained, which was statistically significantly higher than using LiDAR $(65.1 \%)$ or hyperspectral $(69.3 \%)$ data alone. The results of our study demonstrate that plant functional traits retrieved from hyperspectral data using radiative transfer models can be used in conjunction with hyperspectral features and LiDAR metrics to further improve individual tree species classification in a mixed temperate forest.
\end{abstract}




\subsection{Introduction}

Tree species information serves as fundamental data for sustainable forest management (Dalponte et al. 2012; Heinzel and Koch 2012; Kirby and Potvin 2007), ecosystem services assessment (Jones et al. 2010), as well as biodiversity monitoring (Shang and Chazette 2014; Skidmore et al. 2015). Accurately identifying individual tree species is of significance for scientific research and management purposes. Thus, effective and efficient techniques for characterizing and classifying tree species are widely demanded (Lin and Hyyppä 2016).

Compared with conventional field tree species investigation approaches, remote sensing offers a practical and economical means for mapping tree species, especially for large scales (Heinzel and Koch 2012). Multiple types of remotely sensed data, including very high resolution aerial photography (spatial resolution $<50 \mathrm{~cm}$ ), airborne multispectral, hyperspectral and Light Detection and Ranging (LiDAR) data, have been widely used for tree species classifications at the individual tree level (reviewed by Fassnacht et al. (2016)). Combining airborne LiDAR and hyperspectral datasets, as a state-of-the-art remote sensing technology, provides both horizontal and vertical information about tree species and has shown great potential in improving tree species discrimination (Zhang et al. 2016).

The spectral bands reflectance of hyperspectral data, selected using feature reduction methods, have been commonly used in tree species classification (Richter et al. 2016). Spectral vegetation indices, similarly, based on information from a few significant spectral wavelengths, have been employed to measure a range of plant attributes (Liu et al. 2017), such as pigment content in the visible 400-700 nm range (Fassnacht et al. 2015), and water content in near infrared 700-1100 nm range (Liu et al. 2017; Zhang et al. 2015). However, high spectral intraspecies variability, that can exceed the interspecies variability (Debba et al. 2009) due to phenological effects and differences in tree age and health, openness of canopies, shadowing effects, and environment variability (Clark et al. 2005; Waser et al. 2010), restricts tree species separability (Richter et al. 2016).

Airborne LiDAR data represent one of the most accurate methods for measuring structural attributes of trees. LiDAR is a favoured data source for individual tree delineation (Heinzel and Koch 2012; Heurich 2008; Jakubowski et al. 2013; Kim 2007; Vauhkonen et al. 2014), while also providing valuable geometric and radiometric information for tree species discrimination. While the geometric information from LiDAR represents the architecture of crowns, branching, and 
foliage (Coops et al. 2007; Riaño et al. 2004), the radiometric information from the backscattered signal is additionally connected to foliage type (Suratno et al. 2009), leaf size, orientation, and foliage density (Korpela et al. 2010). Suites of those properties extracted from LiDAR point cloud at the individual tree level offer complementary information to the spectral features garnered from hyperspectral data (Alonzo et al. 2014). Increasingly, airborne LiDAR and hyperspectral data have been combined at the pixel or feature level for tree species classification and higher mapping accuracies (compared to using either dataset alone) were reported (e.g. Asner et al. 2008; Dalponte et al. 2008, 2012; Ghosh et al. 2014; Liu et al. 2017; Sommer et al. 2016). However, most of these studies focused on data-driven or algorithm-driven approaches and pursued an optimization of classification accuracy (Fassnacht et al. 2016; Shi et al. 2018b). An in-depth bioecological understanding of the relationship between plant traits and remote sensing observations for tree species classification has not been performed.

From an ecological or biological perspective, classification of individual tree species requires species-specific features, which can be morphological (e.g. tree height, tree branching pattern), physiological (e.g. photosynthesis rate), biochemical (e.g. leaf chlorophyll, leaf water content) or phenological (e.g. leaf phenology, flowering time) features. Tree species mapping with remote sensing should be linked to an understanding that tree species have unique structural profiles and spectral signatures as well as genetic characteristic properties (Asner et al. 2009; Cho et al. 2010; Clark et al. 2005). One way to reconcile the dissimilarity between tree species is by collecting information on their functional traits (Lefcheck et al. 2015). For instance, effective water thickness represents approximately $66 \%$ of leaf fresh mass averaged over a large number of leaf types (Jacquemoud et al. 1996). Leaf mass per area is mainly composed of cellulose, lignin, protein, starch and minerals which related to strategies for assimilation, respiration and evapotranspiration, hence relevant for modelling carbon cycle, identification of functional types, seasonality or leaf turnover rate (Kokaly et al. 2009).

During the past few decades, the capabilities of remote sensing to determine plant functional traits have been established, primarily due to improvements in 400$2500 \mathrm{~nm}$ imaging spectrometers (Asner and Martin 2009; Martin et al. 2018; Milton et al. 2009; Townsend et al. 2003). The spectral separability of species depends, in large part, on the chemical attributes of the leaves, which have already proven to be taxonomically organized (Asner and Martin 2011; Féret and Asner 
2011). Previous studies have indicated that tree species vary markedly in leaf water content (Huber et al. 2008), leaf mass per area (Casas et al. 2014), nitrogen and carbon concentration (Huber et al. 2008; Wang et al. 2016). Based on the data collected in a highly diverse tropical forest, Asner and Martin (2009) found that spectral signatures linked to seven leaf functional traits (chlorophyll a, chlorophyll b, carotenoids, nitrogen, phosphorus, water content, and specific leaf area) are unique for 41 species. Afterwards, Asner and Martin (2011) well discriminated more than 300 Amazon rainforest canopy species by using 11 plant functional traits derived from leaf spectra. These findings showed that there might be potential to develop a phylogeny to estimate plant functional traits, thereby allowing for the improved discrimination of tree species. However, most studies focus on effectively quantifying plant functional traits composition (Clevers et al. 2010; Huber et al. 2008; Kokaly et al. 2009), evaluating approaches for estimating plant functional traits (Ali et al. 2016b; Sehgal et al. 2016; Zhao et al. 2013), or linking related spectra bands and indices to specific plant functional traits (Carter and Knapp 2001; Feilhauer et al. 2015). Very few studies used retrieved plant functional traits in the context of individual tree species classification (Féret and Asner 2011). Although some studies have successfully retrieved plant functional traits in natural forests using physical or empirical approaches (e.g. Ali et al. 2016a; Ali et al. 2016b; Essery et al. 2008; Kötz et al. 2004; Wang et al. 2015), the role of plant functional traits retrieved from hyperspectral data for tree species classification still remains underexplored.

Here, the main objective is to evaluate the capacity of combining plant functional traits retrieved from radiative transfer models (RTMs) with airborne LiDARderived metrics and spectral features generated from hyperspectral data for mapping five tree species in a natural forest in Germany. Specifically, we aim to: (1) generate geometric and radiometric metrics from airborne LiDAR data as well as spectral features from hyperspectral data, (2) retrieve three plant functional traits from airborne hyperspectral data using the INFORM model, (3) combine plant functional traits, spectral features and LiDAR metrics for tree species classification, and (4) evaluate the capability of plant functional traits for discriminating individual tree species. 


\subsection{Materials and Methods}

\subsubsection{Study area}

The study area is located in the Bavarian Forest National Park $\left(49^{\circ} 3^{\prime} 19^{\prime \prime} \mathrm{N}\right.$, $\left.13^{\circ} 12^{\prime} 9^{\prime \prime} \mathrm{E}\right)$, a mixed temperate forest situated in the south-eastern part of Germany (Fig. 4.1) (Shi et al. 2018b). The park covers a total area of 24218 hectares with an elevation varying from $600 \mathrm{~m}$ to $1452 \mathrm{~m}$. The climate of the region is temperate, with a total annual precipitation between $1200 \mathrm{~mm}$ and 1800 $\mathrm{mm}$ and an average annual temperature between $3^{\circ} \mathrm{C}$ and $6^{\circ} \mathrm{C}$. The dominant tree species include Norway spruce (Picea abies), European beech (Fagus sylvatica), and silver fir (Abies alba). Rare species such as sycamore maple (Acer pseudoplatanus), white birch (Betula pendula), common rowan (Sorbus aucuparia), European aspen (Populus tremula), and European ash (Fraxinus excelsior) are also found in the mixed forests (Heurich and Neufanger 2005).

Two species-rich sites (approximately 25 hectares for each site) were identified as study areas of interests in this study (Fig. 4.1). The elevation of two study sites is $675-732 \mathrm{~m}$ and $845-906 \mathrm{~m}$ with a slope of $9.12 \pm 5^{\circ}$ and $8.76 \pm 3^{\circ}$, for site $\mathrm{A}$ and site $\mathrm{B}$, respectively. According to the land cover and land use data provided by Department of Conservation and Research, Bavarian Forest National Park, Site A is dominated by mature coniferous and mixed stands with partly mature and medium deciduous stands. Site B mainly consists of mature deciduous and mixed stands. 


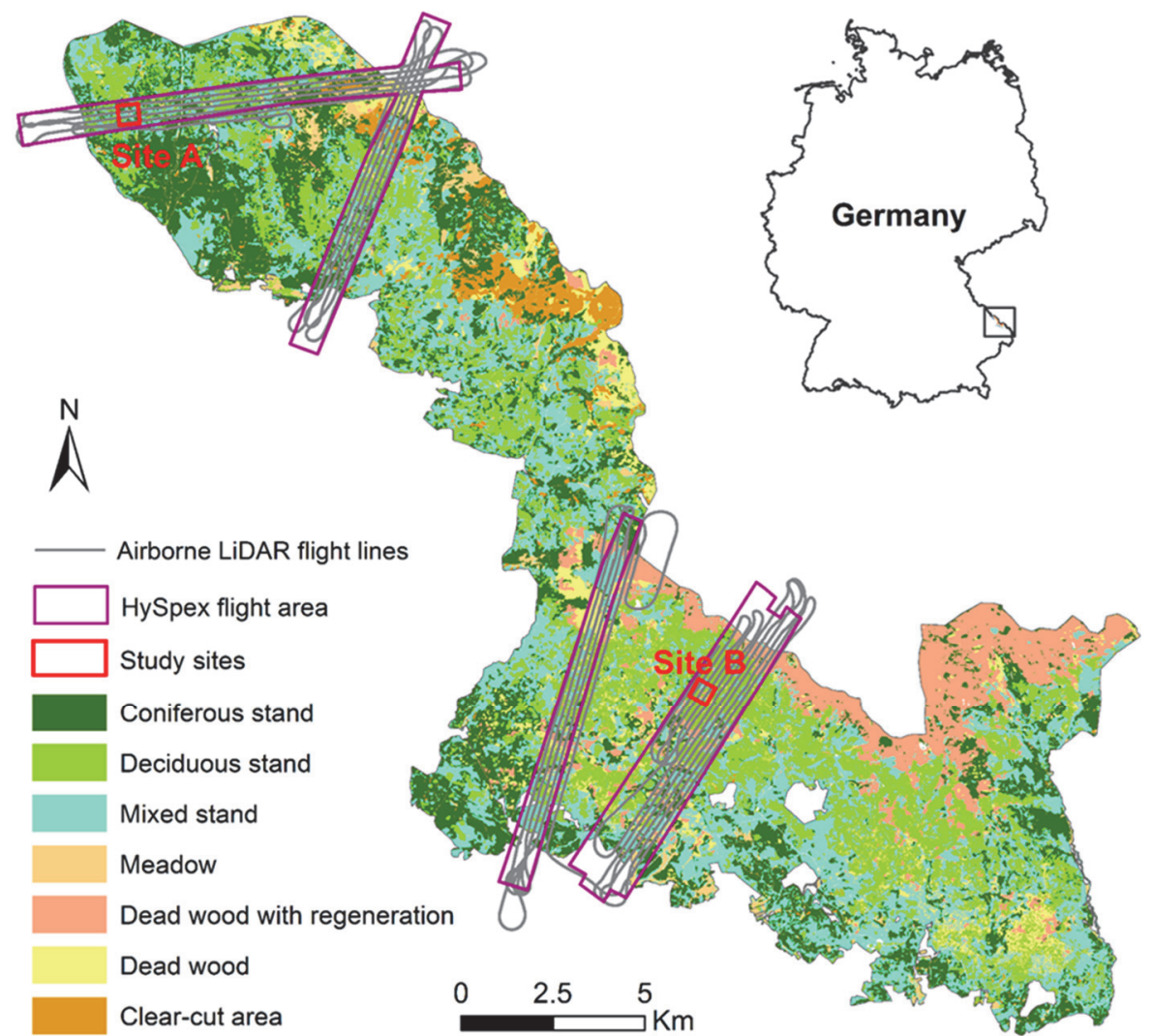

Fig. 4.1 Airborne LiDAR and HySpex data collections and the location of the two study sites in the Bavarian Forest National Park.

\subsubsection{Data acquisition and pre-processing}

\subsubsection{LiDAR data}

The airborne LiDAR data were acquired on 18 August 2016 using the Riegl LMSQ680i scanner operating at a wavelength of $1550 \mathrm{~nm}$. The mean flight speed was $50 \mathrm{~ms}^{-1}$ with a pulse repetition frequency of $400 \mathrm{kHz}$. The flying altitude was approximately $300 \mathrm{~m}$ above ground with a scan angle around $\pm 15^{\circ}$ (see Chapter 3 , section 3.2.3 for a detailed description). 


\subsubsection{Hyperspectral data}

The hyperspectral data were obtained with the HySpex sensor developed by the Norwegian Norsk Elektro Optikk (NEO) company. The HySpex sensor consists of two imaging spectrometers covering spectral ranges of 400-992 nm (VNIR) and 968-2498 nm (SWIR). The HySpex sensor comprises 160 and 256 spectral channels with spectral resolution of $3.6 \mathrm{~nm}$ and $6 \mathrm{~nm}$, and spatial resolution of $1 \mathrm{~m}$ and $2 \mathrm{~m}$, for VNIR and SWIR, respectively.

The HySpex instrument was flown over four transects in the park (Fig. 4.1) on 25 August 2016 on board the Cessna 208B Grand Caravan at an average altitude of $3010 \mathrm{~m}$ above sea level (Köhler 2016). The HySpex data were supplied by the DLR (German Aerospace Centre) team after atmospheric correction performed with the ATCOR4 model, orthorectified and georeferenced using standard aircraft in-flight information such as attitude and flight path data in combination with a digital terrain model (Müller et al. 2005; Richter and Schläpfer 2002; Schwind et al. 2014). Savitzky-Golay filter was applied ( 5 by 5 window size) to correct for random and systematic noise (Schläpfer and Richter 2011). The number of bands was reduced to 290, as bands affected by strong noise or atmospheric effects (water vapour absorption) were eliminated. In detail, the following bands were removed: $887-1000 \mathrm{~nm}, 1093-1189 \mathrm{~nm}, 1303-1489 \mathrm{~nm}$ and 1777-2022 nm. Finally, the hyperspectral data were registered to LiDAR data based on about 40 ground control points for each study site using polynomial warp method (2 degree) and nearest neighbour resampling method in the ENVI software (version 5.2). The overall accuracy of geometric correction was higher than $0.25 \mathrm{~m}$.

\subsubsection{Tree species and plant functional traits measurement}

Fieldwork was carried out in July 2017, including (1) the measurement of the spatial location of individual trees and species identification, (2) the collection of leaf samples from recorded trees, and (3) the measurement of leaf parameters in a local laboratory. We collected the spatial location of individual trees using a Leica Viva GS10 Plus differential GPS (Leica Geosystems AG, Switzerland). After post-processing the raw GPS data, differentially corrected coordinates were obtained with an accuracy higher than $0.50 \mathrm{~m}$. In total, 115 locations of trees at site $\mathrm{A}$ and 107 locations of trees at site $\mathrm{B}$ were collected, including 57 beech trees, 38 birch trees, 41 fir trees, 40 maple trees and 46 spruce trees. Once the location of a tree recorded, mature top-of-canopy leaf samples were collected from at least two sunlit branches within each tree crown, using a crossbow or an extended pole 
pruner when necessary to ensure proper collection. Once acquired, each sample was immediately placed in a zip-locked plastic bag together with wet pulp paper and stored in a portable cooler with frozen icepacks until being transported to the local laboratory for processing within 4-6 $\mathrm{h}$ of the initial collection.

We followed the protocol for standardized measurement of plant functional traits by Cornelissen et al. (2003). The fresh weight of each sample was determined by using a digital scale of high precision. Leaf area of the samples was measured using AM350 Portable Leaf Area Meter. Specifically, Norway spruce needles are cylindrical and therefore their total surface was first computed as a projected area multiplied by a universal conversion factor of 2.57 derived experimentally (Waring 1983). Then, the total needle surface area was divided by two to acquire the hemispherical-surface projection of sampled spruce needles (Ali et al. 2017). Leaf chlorophyll (Cab) was measured by CCM-300 Chlorophyll Content Meter. Finally, scanned leaves were oven dried at $65^{\circ} \mathrm{C}$ for $72 \mathrm{~h}$ and then equivalent water thickness $(\mathrm{Cw})$ and leaf mass per area $(\mathrm{Cm})$ were computed based on fresh and oven-dried leaf mass and leaf areas. The summary statistics of three plant functional traits measured in the laboratory are presented in Table 4.1.

Table 4.1 Summary of the sample trees and three plant functional traits measured in site $\mathrm{A}$ and site B: equivalent water thickness $(\mathrm{Cw})$, leaf mass per area $(\mathrm{Cm})$, leaf chlorophyll (Cab).

\begin{tabular}{|c|c|c|c|c|c|c|c|c|c|c|}
\hline \multirow[b]{2}{*}{$\begin{array}{l}\text { Tree } \\
\text { species }\end{array}$} & \multirow[b]{2}{*}{$\begin{array}{l}\text { Sample } \\
\text { size }\end{array}$} & \multicolumn{2}{|l|}{$\mathrm{CW}(\mathrm{cm})$} & \multirow[b]{2}{*}{ Max } & \multicolumn{2}{|c|}{$\mathrm{Cm}\left(\mathrm{g} \mathrm{cm}^{-2}\right)$} & \multirow[b]{2}{*}{ Max } & \multicolumn{2}{|c|}{$\mathrm{Cab}\left(\mu \mathrm{g} \mathrm{cm}^{-2}\right)$} & \multirow[b]{2}{*}{ Max } \\
\hline & & $\begin{array}{l}\text { Mean } \\
\text { (sd) }\end{array}$ & Min & & $\begin{array}{l}\text { Mean } \\
\text { (sd) }\end{array}$ & Min & & $\begin{array}{l}\text { Mean } \\
\text { (sd) }\end{array}$ & Min & \\
\hline Beech & 57 & $\begin{array}{l}0.0067 \\
(0.0013)\end{array}$ & 0.0044 & 0.0107 & $\begin{array}{l}0.0059 \\
(0.0017)\end{array}$ & 0.0027 & 0.0112 & $\begin{array}{l}41.4 \\
(4.2)\end{array}$ & 31.5 & 49.8 \\
\hline Birch & 38 & $\begin{array}{l}0.0098 \\
(0.0010)\end{array}$ & 0.0081 & 0.0133 & $\begin{array}{l}0.0061 \\
(0.0013)\end{array}$ & 0.0041 & 0.0097 & $\begin{array}{l}38.6 \\
(4.5)\end{array}$ & 30.0 & 50.4 \\
\hline Fir & 41 & $\begin{array}{l}0.0166 \\
(0.0012)\end{array}$ & 0.0139 & 0.0209 & $\begin{array}{l}0.0141 \\
(0.0018)\end{array}$ & 0.0115 & 0.0177 & $\begin{array}{l}56.3 \\
(11.0)\end{array}$ & 38.1 & 74.5 \\
\hline Maple & 40 & $\begin{array}{l}0.0095 \\
(0.0012)\end{array}$ & 0.0071 & 0.0120 & $\begin{array}{l}0.0060 \\
(0.0013)\end{array}$ & 0.0033 & 0.0086 & $\begin{array}{l}43.3 \\
(5.3)\end{array}$ & 25.7 & 51.6 \\
\hline Spruce & 46 & $\begin{array}{l}0.0140 \\
(0.0024)\end{array}$ & 0.0106 & 0.0206 & $\begin{array}{l}0.0103 \\
(0.0017)\end{array}$ & 0.0074 & 0.0157 & $\begin{array}{l}34.0 \\
(6.4)\end{array}$ & 22.2 & 46.4 \\
\hline
\end{tabular}

\subsubsection{Individual tree segmentation}

We used an adapted 3D segmentation algorithm proposed by Yao et al. (2013) to automatically extract individual trees from airborne LiDAR data (see chapter 2, section 2.2.3 for a detailed description). We linked the sample trees to the correct LiDAR segmentation results with the assistance of a very high resolution aerial photograph ( $0.25 \mathrm{~m}$ spatial resolution) using the procedures we proposed in Chapter 2. As a result, 215 sample trees (including 55 beech trees, 37 birch trees, 40 fir trees, 38 maple trees and 45 spruce trees) from site A and site B were 
selected for further analysis. The cloud points within each correct segments were extracted and assigned to the corresponding sample trees for the derivation of LiDAR metrics.

\subsubsection{Feature derivation}

\subsubsection{LiDAR metrics derivation}

Based on the LiDAR metrics derivation from Chapter 2, forty commonly used LiDAR metrics were extracted from each tree segment and classified into two categories: geometric metrics (e.g. tree height, crown shape and crown volume) and radiometric metrics (e.g. intensity and echo width). Statistical parameters (i.e. maximum, mean, standard deviation, coefficient variation, skewness, kurtosis, variance, 25th percentile and 90th percentile) of the height, intensity and echo width values of the laser points within a tree crown were calculated respectively. Point distribution metrics (i.e. the percentage of first return to all returns, the percentage of last returns to all returns, first returns above mean height, and all returns above $2 \mathrm{~m}$ ) and crown shape metrics (i.e. the ratio of crown base height to tree height, the ratio of crown volume to crown area, and canopy relief ratio) were employed. Additionally, the value of height, intensity and echo width with respect to two different echo categories: "first-or-single returns" and "single returns" were also generated from each segment (Hovi et al. 2016; Ørka et al. 2010; Shi et al. 2018b). Height related LiDAR metrics were normalized by tree height to eliminate scale dependency. The derivation of LiDAR metrics was conducted in $\mathrm{R}$ language environment (http://www.r-project.org/).

\subsubsection{Spectral features derivation}

Due to issues such as shadowing as well as errors in individual tree crown delineation, it has often been advantageous to use only sunlit pixels for plant functional traits retrieval (Ali et al. 2017; Martin et al. 2018) as well as for tree species classification (Clark et al. 2005; Dalponte et al. 2013; Ghosh et al. 2014; Immitzer et al. 2012; Richter et al. 2016). For hyperspectral data, we therefore manually selected the sunlit pixels within each tree crown and only the spectral information of selected pixels was considered for further analysis. For each tree crown, we first overlaid the tree top derived from LiDAR data with the hyperspectral image, then based on the size of the tree crown we chose 1-4 sunlit pixels centred by the tree top to represent the tree (Fig. 4.2). The selected pixels within each crown were assigned to the sample tree and were further used for 
extracting spectral features as well as for retrieving plant functional traits (Asner 2007).

The mean value of spectral reflectance of each waveband (B1, B2, .., B290) and the first derivation of each waveband (D1, D2, ..., D290) were calculated from selected pixels within each crown. We computed 27 vegetation indices from the VNIR and SWIR regions of the spectrum based on previous research (Table 4.2).

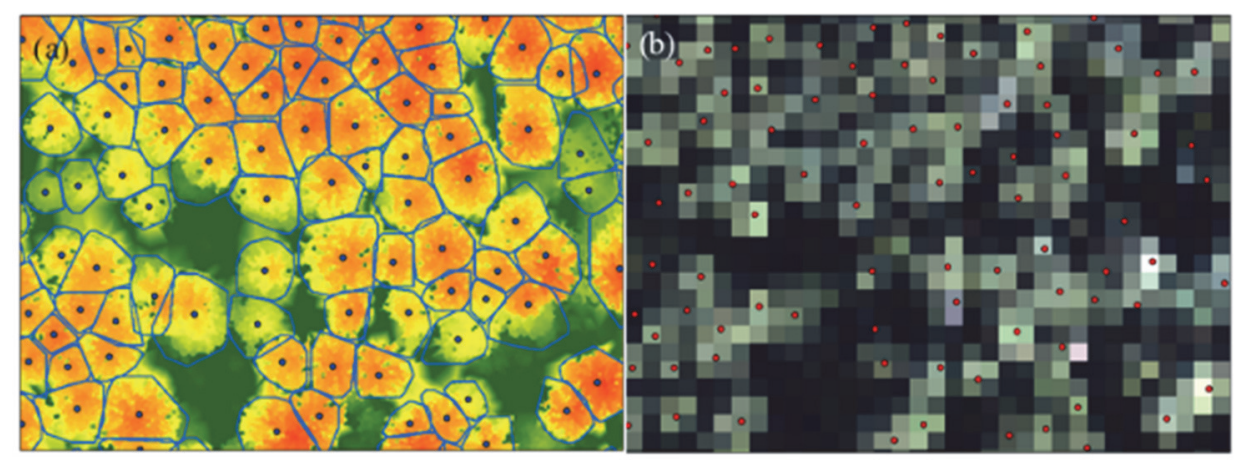

Fig. 4.2 3D individual tree segmentation using the approach of Yao et al. (2013) on (a) CHM with tree crown and tree top derived from LiDAR data, and (b) HySpex data with tree top derived from LiDAR data. 
Table 4.2 Description of generated hyperspectral features

\begin{tabular}{|c|c|c|c|}
\hline Variables & Index or description & Formula & Reference \\
\hline \multicolumn{4}{|l|}{ Band reflectance } \\
\hline B1-B290 & $\begin{array}{l}\text { Band reflectance from 415.7-2496.5 } \\
\mathrm{nm}\end{array}$ & & \\
\hline \multicolumn{4}{|l|}{ First derivation } \\
\hline D1-D290 & First derivation of band reflectance & & \\
\hline \multicolumn{4}{|c|}{ Vegetation indices } \\
\hline ACI2 & Anthocyanin Content Index & $\mathrm{ACI} 2=\rho 650 / \rho 550$ & (Gamon and Surfus 1999) \\
\hline Carter5 & Ratio vegetation stress index & Carter $5=\rho 695 / \rho 670$ & (Carter 1994) \\
\hline CI & Chlorophyll Index & $\mathrm{CI}=\rho 675^{*} \rho 690 / \rho 683^{2}$ & $\begin{array}{l}\text { (Zarco-Tejada et al. } \\
\text { 2000) }\end{array}$ \\
\hline $\mathrm{CI} 2$ & Chlorophyll Index & CI $2=\rho 760 / \rho 700-1$ & (Gitelson et al. 2003a) \\
\hline CRI2 & Chlorophyll Reciprocal Index & $\mathrm{CRI} 2=1 / \rho 515-1 / \rho 770$ & (Gitelson et al. 2003a) \\
\hline Datt & Chlorophyll content index & Datt $=(\rho 850-\rho 710) /(\rho 850-\rho 680)$ & (Datt 1999) \\
\hline DWSI1 & Disease-Water Stress Index & DWSI $1=\rho 800 / \rho 1660$ & (Apan et al. 2004) \\
\hline DWSI2 & Disease-Water Stress Index & DWSI $2=\rho 1660 / \rho 550$ & (Apan et al. 2004) \\
\hline DWSI5 & Disease-Water Stress Index & DWSI5 $=(\rho 800+\rho 550) /(\rho 1660+\rho 680)$ & (Apan et al. 2004) \\
\hline Green_NDVI & $\begin{array}{l}\text { NDVI in the green and red edge } \\
\text { regions }\end{array}$ & $\begin{array}{l}\text { Green_NDVI }=(\rho 800- \\
\rho 550) /(\rho 800+\rho 550)\end{array}$ & (Gitelson et al. 2003b) \\
\hline LWVI-1 & Leaf Water Vegetation Index & LWVI-1=( $(1094-\rho 983) /(\rho 1094+\rho 983)$ & (Galvão et al. 2005) \\
\hline Mean_B690_B7 & $\begin{array}{l}\text { The average reflectance between } 690 \\
\mathrm{~nm} \text { to } 740 \mathrm{~nm}\end{array}$ & & (Merton 1999) \\
\hline MNDVI & $\begin{array}{l}\text { Modified Normalized Difference } \\
\text { Vegetation Index }\end{array}$ & $\begin{array}{l}\text { MNDVI }=(\rho 800-\rho 680) /(\rho 800+\rho 680- \\
\left.2^{*} \rho 445\right)\end{array}$ & (Sims and Gamon 2002) \\
\hline MPRI & $\begin{array}{l}\text { Modified Photochemical Reflectance } \\
\text { Index }\end{array}$ & MPRI $=(\rho 515-\rho 530) /(\rho 515+\rho 530)$ & $\begin{array}{l}\text { (Hernández-Clemente et } \\
\text { al. 2011) }\end{array}$ \\
\hline NDVI & $\begin{array}{l}\text { Normalized Difference Vegetation } \\
\text { Index }\end{array}$ & $\mathrm{NDVI}=(\rho 800-\rho 670) /(\rho 800+\rho 670)$ & (Rouse Jr et al. 1974) \\
\hline PRI & Photochemical reflectance index & $P R I=(\rho 531-\rho 570) /(\rho 531+\rho 570)$ & (Gamon et al. 1995) \\
\hline PSI & Plant Stress Index & $\mathrm{PSI}=\rho 695 / \rho 760$ & (Carter and Miller 1994) \\
\hline PSSR1 & Pigment Specific Simple Ratio & PSSR $1=\rho 800 / \rho 680$ & (Blackburn 1998) \\
\hline PSSR2 & Pigment Specific Simple Ratio & $\operatorname{PSSR} 2=\rho 800 / \rho 635$ & (Blackburn 1998) \\
\hline R1 & Ratio vegetation stress index & $\mathrm{R} 1=\rho 694 / \rho 760$ & (Carter and Miller 1994) \\
\hline R2 & Ratio vegetation stress index & $\mathrm{R} 2=\rho 600 / \rho 760$ & (Carter and Miller 1994) \\
\hline R3 & Ratio vegetation stress index & $\mathrm{R} 3=\rho 710 / \rho 760$ & (Carter and Miller 1994) \\
\hline RVSI & Red-edge Vegetation Stress Index & RVSI $=(\rho 714+\rho 752) / 2-\rho 733$ & (Merton 1998) \\
\hline SL & Slope of red-edge & $\mathrm{SL}=(\rho 740-\rho 690) / \mathrm{N} 740-690$ & (Merton 1998) \\
\hline SWIR_VI & Shortwave-Infrared Vegetation Index & $\begin{array}{l}\text { SWIR_VI=37.72*( }(\rho 2210- \\
\rho 2090)+26.27 *(\rho 2280-\rho 2090)+0.57\end{array}$ & (Lobell et al. 2001) \\
\hline TVI & Triangular Vegetation Index & $\begin{array}{l}\text { TVI }=0.5 *(120 *(\rho 2210-\rho 2090)- \\
200 *(\rho 670-\rho 550)\end{array}$ & (Broge and Leblanc 2001) \\
\hline Vogelmann2 & $\begin{array}{l}\text { Ratio vegetation index in red edge } \\
\text { regions }\end{array}$ & Vogelmann $2=(\rho 734-\rho 747) /(\rho 715+\rho 726$ & (Vogelmann et al. 1993) \\
\hline
\end{tabular}

\subsubsection{Retrieval of plant functional traits from hyperspectral imagery using INFORM model}

Two approaches have been developed to estimate plant functional traits from remote sensing data: (i) empirical approach (such as spectral indices and multiple regressions), and (ii) radiative transfer models (RTMs) inversion (Ali et al. 2017). The most commonly used method in the empirical approach is the vegetation index (VI). Vegetation indices (VIs) constitute simple and convenient algebraic combinations of spectral reflectance to extract information from remotely sensed data, which facilitate the processing and analysis of large amounts of remotely sensed data, but they have the limitation of often being site-specific based on a limited number of species, contributing to the diverging forms of indices available in the literature even for the same plant functional traits (Ali et al. 2017; 
Schlerf et al. 2010). RTMs allow the creation of simulated training databases covering a wide range of spectral data to which inversion algorithms such as Look-Up Tables (LUT) inversion and Artificial Neural Network (ANN) can be applied to retrieve parameters from remote sensing data (Ali et al. 2017). Nevertheless, RTMs still require local information, such as cover type, in order to be accurately upscaled (Si et al. 2012).

The invertible forest reflectance model (INFORM) (Atzberger 2000) was chosen for canopy reflectance modeling and inversion. INFORM is a hybrid model with geometrical-optical (crown shadowing) and turbid-medium (multiple scattering) representations (Schlerf and Atzberger 2006). The choice of INFORM was a trade-off between suitability for forest structure characterization and simplicity in generating scenes and inversion (Schlerf and Atzberger 2012). The ranges of $\mathrm{Cw}, \mathrm{Cm}$ and $\mathrm{Cab}$ were set based on the field data, while the solar zenith angle, observation angle and azimuth angle were determined based on the HySpex acquisition metadata. The ranges of stem density ( $\mathrm{SD}, \mathrm{n} / \mathrm{ha}$ ), stand height ( $\mathrm{SH}$, $\mathrm{m}$ ), crown diameter $(\mathrm{CD}, \mathrm{m})$ and average leaf angle (ALA, degree) were decided based on the field measurement (Ali et al. 2016b; Wang et al. 2018). The measured background reflectance was introduced as psoil (Atzberger 2000). Other leaf, canopy, and external input parameters were selected similarly in agreement with the existing literature (e.g. Ali et al. 2016b; Casas et al. 2014; Clevers et al. 2010; Schlerf and Atzberger 2006; Verhoef and Bach 2007). Input parameters and ranges are shown in Table 4.3.

A Look-Up Table (LUT), containing 100,000 randomly generated (uniform distributions) parameter combinations, was generated from the forward calculation of the INFORM model. To find the solution to the inversion for a given canopy spectrum for each estimated reflectance spectrum of the LUT, the $R M S E$ between measured and estimated spectra was calculated according to:

$$
R M S E=\sqrt{\frac{\sum_{i=1}^{n}\left(R_{\text {measured }_{\lambda}}-R_{L U T_{\lambda}}\right)^{2}}{n}}
$$

where $R_{\text {measured }}$ is a measured reflectance at wavelength $\lambda$ and $R_{L U T_{\lambda}}$ is an estimated reflectance at wavelength $\lambda$ in the LUT, and $n$ is the number of wavelengths (Darvishzadeh et al. 2008). To enhance the consistency of the estimated variables, we used the mean value of the best 100 simulations as the final parameter combination (Sehgal et al. 2016). 
Table 4.3 Input parameters and ranges used for generating the LUT from the INFORM model

\begin{tabular}{llll}
\hline & Parameters & Unit & Range \\
\hline $\mathrm{N}$ & Structure parameter & - & $1.5-2.5$ \\
$\mathrm{Cab}$ & Leaf chlorophyll & $\mu \mathrm{g} \mathrm{cm}^{-2}$ & $20-80$ \\
$\mathrm{Cm}$ & Leaf mass per area & $\mathrm{g} \mathrm{cm}^{-2}$ & $0.003-0.030$ \\
$\mathrm{CW}$ & Equivalent water thickness & $\mathrm{cm}$ & $0.005-0.035$ \\
$\mathrm{LAI}_{\mathrm{s}}$ & Single tree LAI & - & $2-8$ \\
$\mathrm{LAI}_{\mathrm{u}}$ & Understory LAI & - & $0-1$ \\
$\mathrm{SD}$ & Stand density & $\mathrm{ha}^{-1}$ & $400-1800$ \\
$\mathrm{SH}$ & Stand height & $\mathrm{m}$ & $5-45$ \\
$\mathrm{CD}$ & Crown diameter & $\mathrm{m}$ & $3-11$ \\
$\mathrm{ALA}$ & Average leaf angle & degree & $20-70$ \\
$\theta_{\mathrm{s}}$ & Sun zenith angle & degree & $45-49$ \\
$\theta_{\mathrm{o}}$ & Observation zenith angle & degree & $20-50$ \\
$\Psi$ & Azimuth angle & degree & $120-150$ \\
Skyl & Fraction of diffused radiation & - & 0.1 \\
\hline
\end{tabular}

\subsubsection{Feature selection and classification algorithm}

Prior knowledge regarding important LiDAR metrics and the useful spectral features for species discrimination was considered during feature selection. Chapter 2 evaluated the performance of 37 commonly used LiDAR metrics for tree species discrimination in the same study area, which provided a solid foundation for the selection of LiDAR metrics in the current study. We also referred previous studies on tree species classification using hyperspectral data for the selection of species-related spectral bands and derivations as well as commonly used vegetation indices (e.g. Ali et al. 2017; Dalponte et al. 2012; Liu et al. 2017; Sommer et al. 2016; Wang et al. 2016), among which Ali et al. (2017), Wang et al. (2016) and Sommer et al. (2016) also conducted their experiments in Bavarian Forest National Park on the similar tree species.

The Random Forest algorithm (Breiman 2001) was used to assess the performance of input variables and classify tree species. It utilizes a composite set of decision trees and can be used to select and rank the predictor variables according to their ability to discriminate between the target classes (Liu et al. 2017). The ability of handling a large number of input variables and reducing overfitting makes the Random Forest algorithm preferable to other classifiers when mixed sets of input variables (e.g. spectral, texture, geometric, indices) are 
integrated into classification (Fassnacht et al. 2016). The Mean Decrease Accuracy (MDA) index of each variable is determined during the out-of-bag error calculation. The greater the decrease in accuracy of the classification due to the exclusion of a single variable, the more important that variable is to the model, therefore a higher MDA value indicates a variable is more important than others (Immitzer et al. 2012; Liu et al. 2017). The procedure of feature selection was conducted by the following steps: firstly, we classified tree species using LiDAR metrics and hyperspectral features alone, and ranked the input variables by their importance indicated by MDA index, respectively; secondly, we calculated the accumulated contribution rate which varied by increasing the number of input variables for classification and we then retained the variables which reached the highest accuracy during classification; finally, 12 LiDAR derived metrics and 15 hyperspectral derived spectral features were chosen for the final classification combined with three retrieved plant functional traits.

\subsubsection{Assessing the performance of classification and input variables}

To assess the classification performance with different variable combinations, we used producer's accuracy, user's accuracy, overall accuracy and kappa coefficient (Cohen 1960). We also employed the McNemar's test to determine the significance level between classification results using different variable combinations (de Leeuw et al. 2006; McNemar 1947). The importance of input variables for species classification was assessed using MDA index (Breiman 2001).

\subsection{Results}

\subsubsection{Retrievals of plant functional traits using INFORM}

Fig. 4.3 shows the relation between estimated plant functional traits against the lab measured plant functional traits. The prediction is more accurate for $\mathrm{Cm}\left(\mathrm{R}^{2}\right.$ $=0.7781)$ and $\mathrm{Cab}\left(\mathrm{R}^{2}=0.7624\right)$ than for $\mathrm{Cw}\left(\mathrm{R}^{2}=0.6784\right)$. $\mathrm{Cw}$ is slightly underestimated compared to $\mathrm{Cm}$ and $\mathrm{Cab}$. 

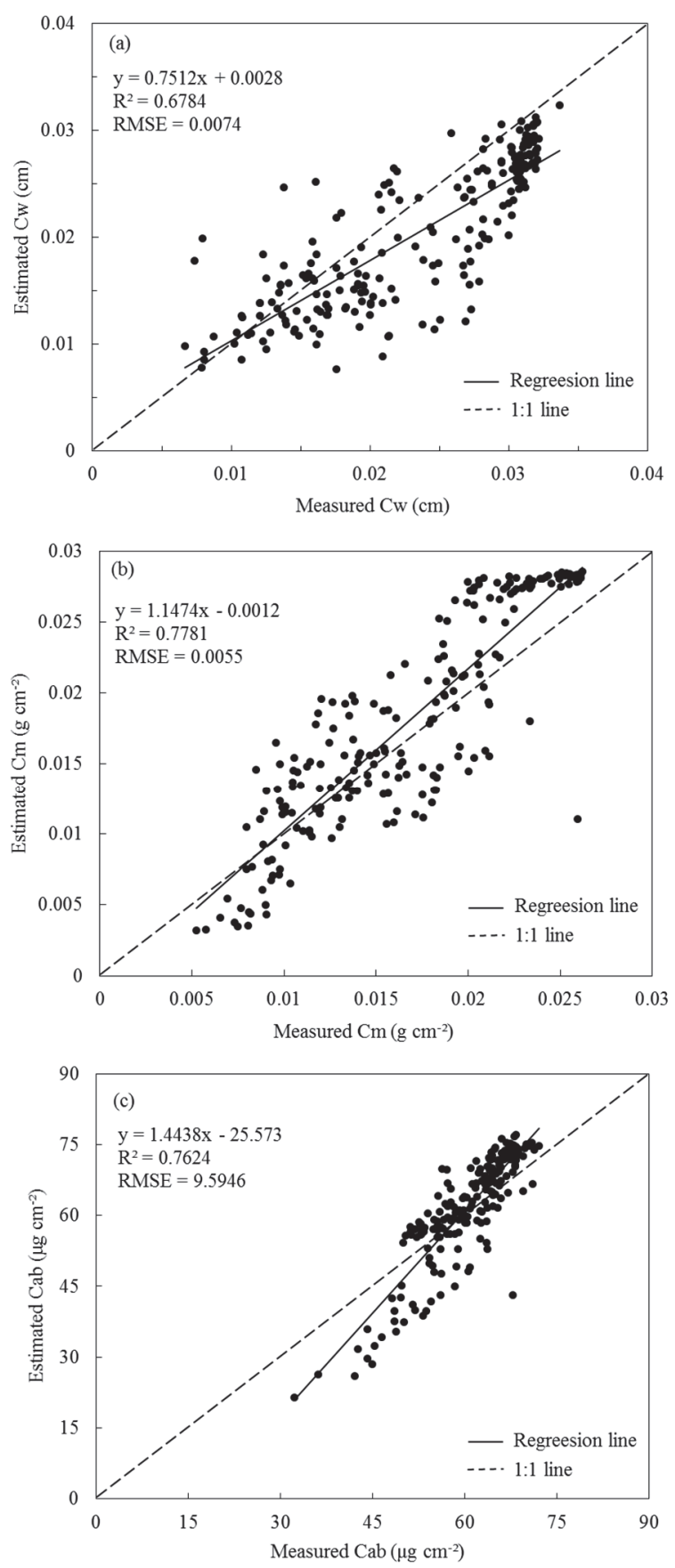

Fig. 4.3 Scatter plots of measured and estimated $\mathrm{Cw}$ (a), Cm (b) and Cab (c) using the INFORM model. Data points are derived from the measured (validation) dataset collected from the study site, and each point represents a sample tree (215 sample trees in total). 


\subsubsection{Feature selection}

Fig. 4.4 shows the average reflectance of the five tree species at $400-2498 \mathrm{~nm}$ wavelengths. The coniferous and deciduous trees had different spectral ranges, while the reflectance variation between deciduous trees was more distinguishable than coniferous trees, especially in the NIR wavelengths and the first peak of SWIR wavelengths. Beech and maple as well as fir and spruce have been identified as two pairs of species with similar spectral signatures, while maple had the highest reflectance variation within the NIR wavelengths compared to other four species. Among the five species analysed, differences in spectral reflectance were most evident in the range of 730-1300 nm as well as 1550-1900 $\mathrm{nm}$. Eventually, 30 variables were selected for tree species classification, including 12 LiDAR derived metrics (LiDAR) and 15 spectral features derived from hyperspectral data (HSI) as well as three retrieved plant functional traits (PFTs) (Table 4.4).
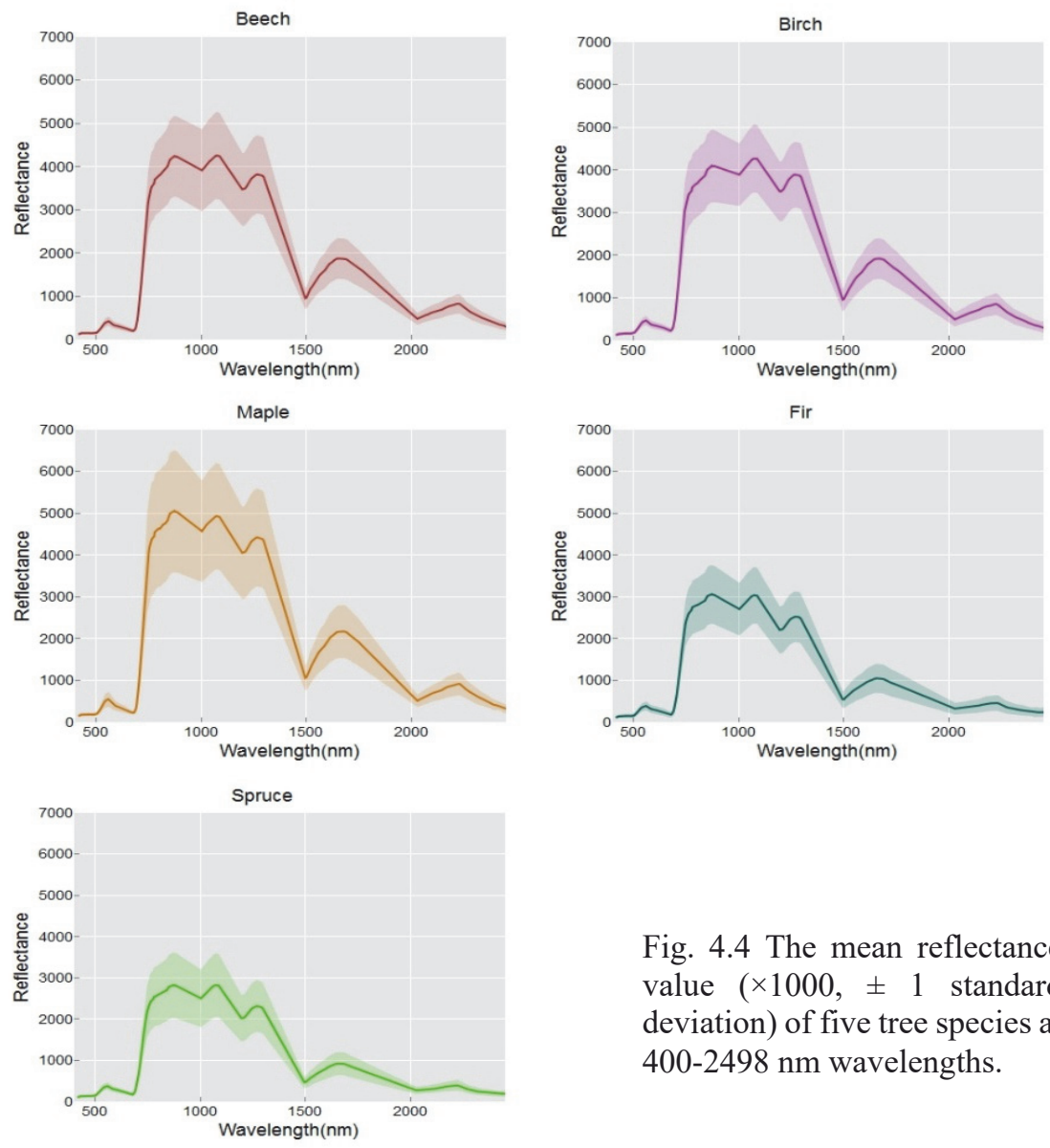
Table 4.4 Selected variables derived from LiDAR and hyperspectral data for classification

\begin{tabular}{ll}
\hline Input variables & Index or description \\
\hline LiDAR metrics (12) & Mean intensity of first-or-only returns \\
Imean_first & Tree height \\
Height & Mean echo width of first-or-single returns \\
Ewmean_first & Mean intensity of single returns \\
Imean_single & Mean echo width of single returns \\
Ewmean_single & 90th percentile of intensity \\
Ip90 & Mean echo width \\
EWmean & Mean intensity \\
Imean & Variation of intensity \\
Ivar & Standard deviation of intensity \\
Isd & Coefficient variation of echo width \\
EWcv & Mean height of single returns \\
Hmean_single & \\
Spectral features (HSI) (15) & D1531.3, D1561.3, D1657.2, D1663.2, D1693.2, \\
First derivation of spectral band & D1771.1, D2034.9, D2082.9 \\
reflectance & B1561.3, B1717.2, B1771.1 \\
Band reflectance (nm) & \\
Vegetation Indices & Anthocyanin content index (ACI2) \\
ACI2 & DWSI2= $1660 / \rho 550$ \\
DWSI2 & SWIR_VI=37.72*( $\rho 2210-\rho 2090)+26.27 *(\rho 2280-$ \\
SWIR_VI & $\rho 2090)+0.57$ \\
RVSI & RVSI= ( $\rho 714+\rho 752) / 2-\rho 733$ \\
Plant functional traits (PFTs) (3) & \\
Cw (cm) & Equivalent water thickness \\
Cm (g cm $\left.{ }^{-2}\right)$ & Leaf mass per area \\
Cab ( $\mu g$ cm $\left.{ }^{-2}\right)$ & Leaf chlorophyll \\
\hline
\end{tabular}

\subsubsection{Comparison of classification accuracies}

Table 4.5 summarizes confusion matrices and producer's and user's classification accuracies for each species using all selected variables. The classification of birch trees obtained both the highest user's and producer's accuracy $(86.8 \%$ and $89.2 \%$, respectively). The lowest user's and producer's accuracies were obtained in the classification of beech and maple, which also had unequal samples ( 55 beech trees and 38 maple trees).

Table 4.6 compares the classification results of different variable combinations. When combined LiDAR, HSI and PFTs variables, the best overall classification accuracy of $83.7 \%$ with 0.74 kappa coefficient was obtained. The classification using LiDAR + HSI and LiDAR + PFTs yielded similar results, with an overall 
accuracy improvement of $11.6 \%$ and $12.6 \%$ from using LiDAR alone, respectively. Moreover, the classification using HSI alone provided a better result than using LiDAR dataset.

Table 4.7 displays the significant levels between classification results generated from different combinations based on $p$ values in McNemar's test. Combining LiDAR, HSI and PFTs variables significantly improved the accuracy from using LiDAR + HSI and LiDAR + PFTs. However, there was no statistically significant difference between the classification performance of using LiDAR + HSI and LiDAR + PFTs, and the same result was found between the use of LiDAR and HSI variables (McNemar's test, $p>0.05$ ).

Table 4.5 Confusion matrix of classification performance

\begin{tabular}{lllllll}
\hline & Beech & Birch & Fir & Maple & Spruce & PA(\%) \\
\hline Beech & 46 & 3 & 0 & 4 & 2 & 83.6 \\
Birch & 2 & 33 & 1 & 1 & 0 & 89.2 \\
Fir & 2 & 1 & 33 & 0 & 4 & 82.5 \\
Maple & 6 & 1 & 0 & 30 & 1 & 78.9 \\
Spruce & 2 & 0 & 4 & 0 & 39 & 86.7 \\
UA(\%) & 79.3 & 86.8 & 86.8 & 85.7 & 84.8 & \\
\hline
\end{tabular}

UA: user's accuracy; PA: producer's accuracy

Table 4.6 The classification results using different sets of variables (LiDAR: LiDAR derived metrics, HSI: spectral features, PFTs: retrieved plant functional traits)

\begin{tabular}{llll}
\hline Dataset & Number of variables & Overall accuracy & Kappa \\
\hline LiDAR+ HSI+ PFTs & 30 & $84.2 \%$ & 0.74 \\
LiDAR+ PFTs & 15 & $77.7 \%$ & 0.70 \\
LiDAR+ HSI & 27 & $76.7 \%$ & 0.69 \\
HSI & 15 & $69.3 \%$ & 0.59 \\
LiDAR & 12 & $65.1 \%$ & 0.57 \\
\hline
\end{tabular}

Table 4.7 McNemar's test for pairwise comparison between classification results generated from different combinations. ${ }^{* *}: p<0.001 ;{ }^{* *}: p<0.01 ;{ }^{*}: p<0.05$; NS: $p>$ 0.05 .

\begin{tabular}{lllll}
\hline & LiDAR + PFTs & LiDAR + HSI & LiDAR & HSI \\
\hline LiDAR + HSI + PFTs & $*$ & $* *$ & $* * *$ & $* * *$ \\
LiDAR + PFTs & & NS & $* * *$ & $* * *$ \\
LiDAR + HSI & & & $* * *$ & $* * *$ \\
LiDAR & & & & NS \\
\hline
\end{tabular}




\subsubsection{Performance of selected features in tree species classification}

Fig. 4.5 presents the relative importance and ranking of the selected variables for the classification using MDA. When combined LiDAR metrics and retrieved plant functional traits, $\mathrm{Cw}$ and $\mathrm{Cm}$ showed greater importance than most LiDAR metrics. Specifically, the top-ranking LiDAR metric is the mean intensity of firstor-single returns (Imean_first) (Fig. 4.5c). When combined LiDAR metrics and spectral features, 7 out of the 10 top-ranked variables were first derivations and vegetation indices, though the best-performing metric was still LiDAR derived (i.e. Imean_first) (Fig. 4.5b).

When combined LiDAR metrics and spectral features with retrieved plant functional traits, the mean intensity of first-or-single returns was the most important variable for species classification, followed by retrieved equivalent water thickness $(\mathrm{Cw})$ and first derivation of band $1771.1 \mathrm{~nm}$. Among the 30 selected variables used for classification, top 10 variables were consisted of 4 LiDAR derived radiometric metrics (i.e. Imean_first, Imean_single, Ip90 and Ewmean), 4 spectral features ( 3 first derivations and 1 vegetation index) and 2 retrieved plant functional traits (i.e. $\mathrm{Cw}$ and $\mathrm{Cm}$ ).

\subsubsection{The capability of metrics for tree species discrimination}

Fig. 4.6 shows differences between the 5 tree species for 6 important variables derived from LiDAR and hyperspectral data. Fig. 4.6a and $\mathrm{b}$ show a distinct difference of equivalent water thickness $(\mathrm{CW})$ and leaf mass per area $(\mathrm{Cm})$ among 5 tree species, which differentiates coniferous trees from deciduous trees. Similarly, the first derivation of band $1771.1 \mathrm{~nm}$ (Fig. 4.6e) and SWIR_VI (Fig. 4.6f) separate coniferous trees from deciduous trees. It is evident that beech and maple have a higher value of the mean intensity of first-or-single returns compared to other species (Fig. 4.6c), which aid discrimination of fir and spruce from beech and maple, as well as birch from other deciduous trees. Furthermore, the mean value of echo width gives a great chance to separate birch from other four species (Fig. 4.6d). 


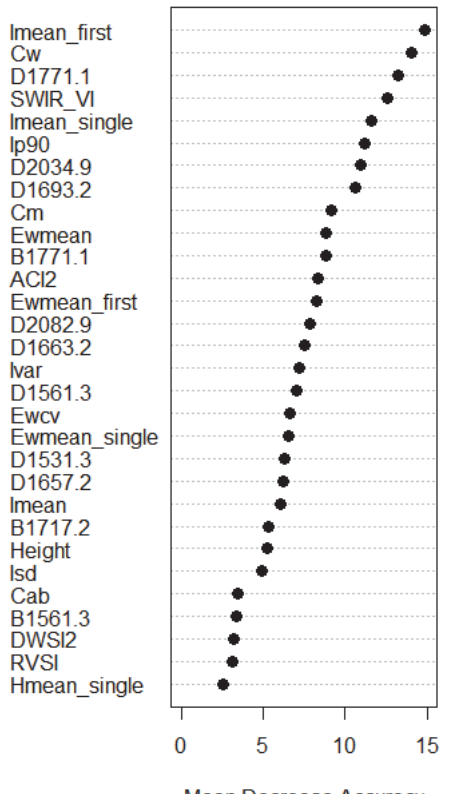

(a) LiDAR+ HSI+ PFTs

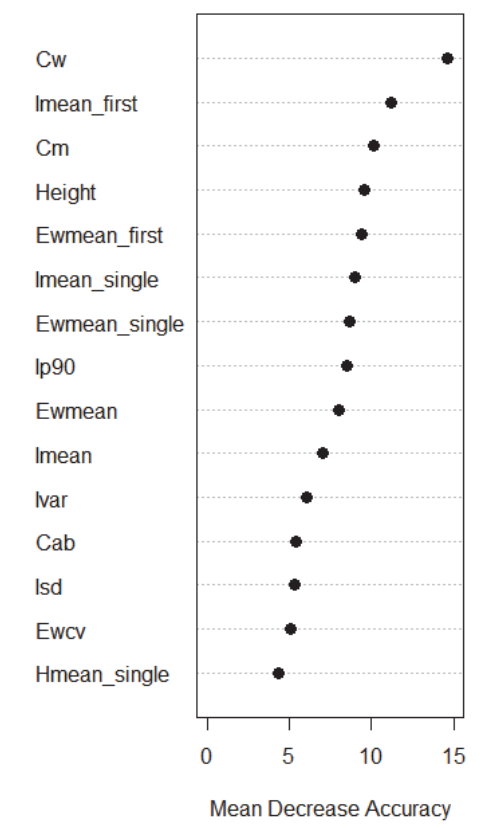

(c) $\mathrm{LiDAR}+$ PFTs

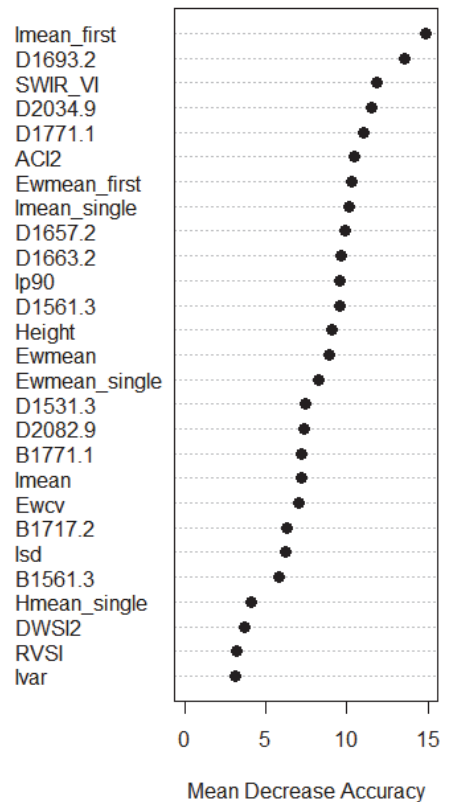

(b) LiDAR+ HIS

Fig. 4.5 The relative importance and ranking of the selected variables for tree species classification under different combinations (a) LiDAR + HSI + PFTs, (b) LiDAR + HSI and (c) LiDAR+ PFTs. 

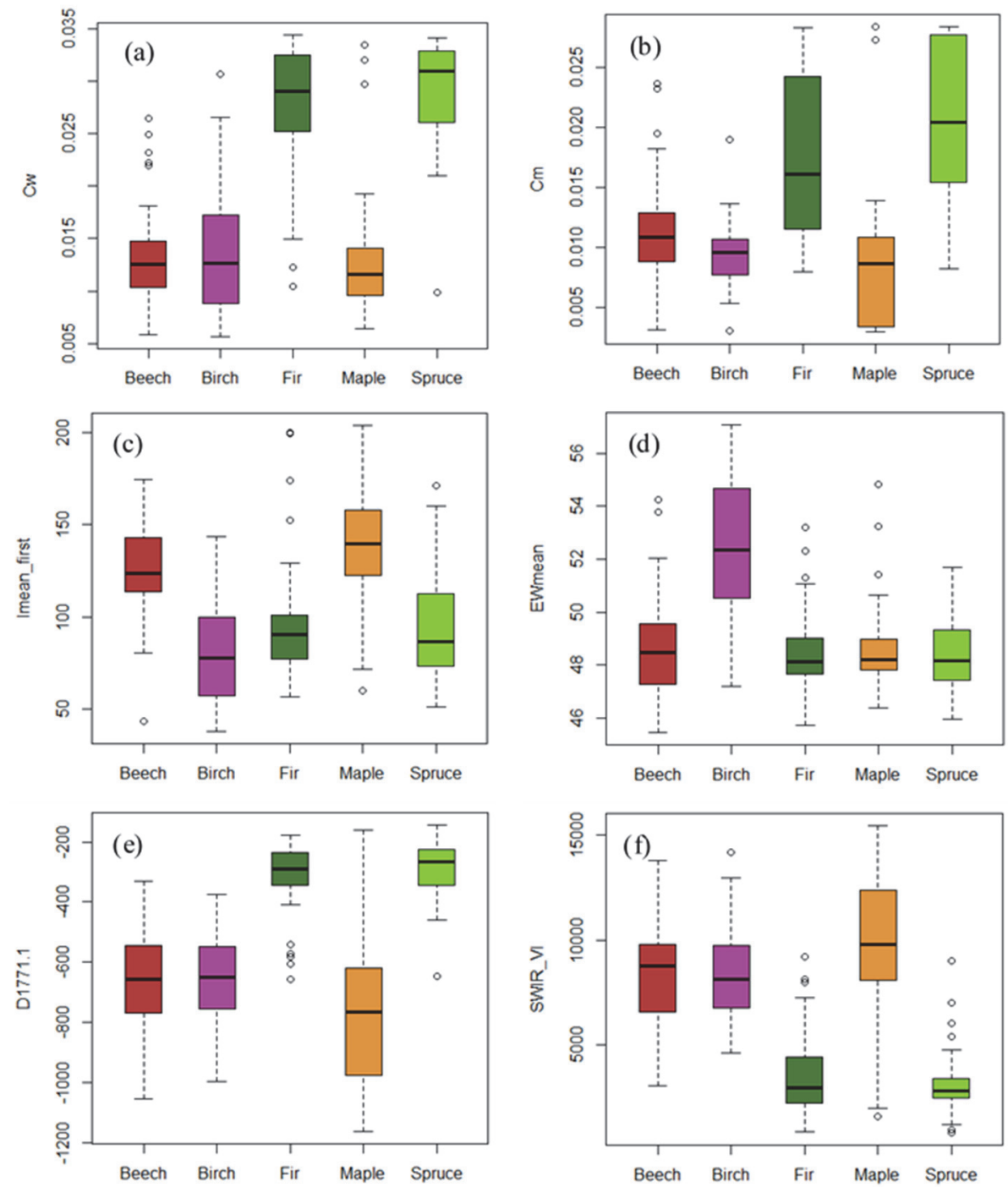

Fig. 4.6 Box plots of equivalent water thickness (a), dry matter content (b), mean intensity of first-or-single returns (c), mean value of echo width (d), first derivation of band 1771.1 nm (e), SWIR_VI (f) among five tree species. 


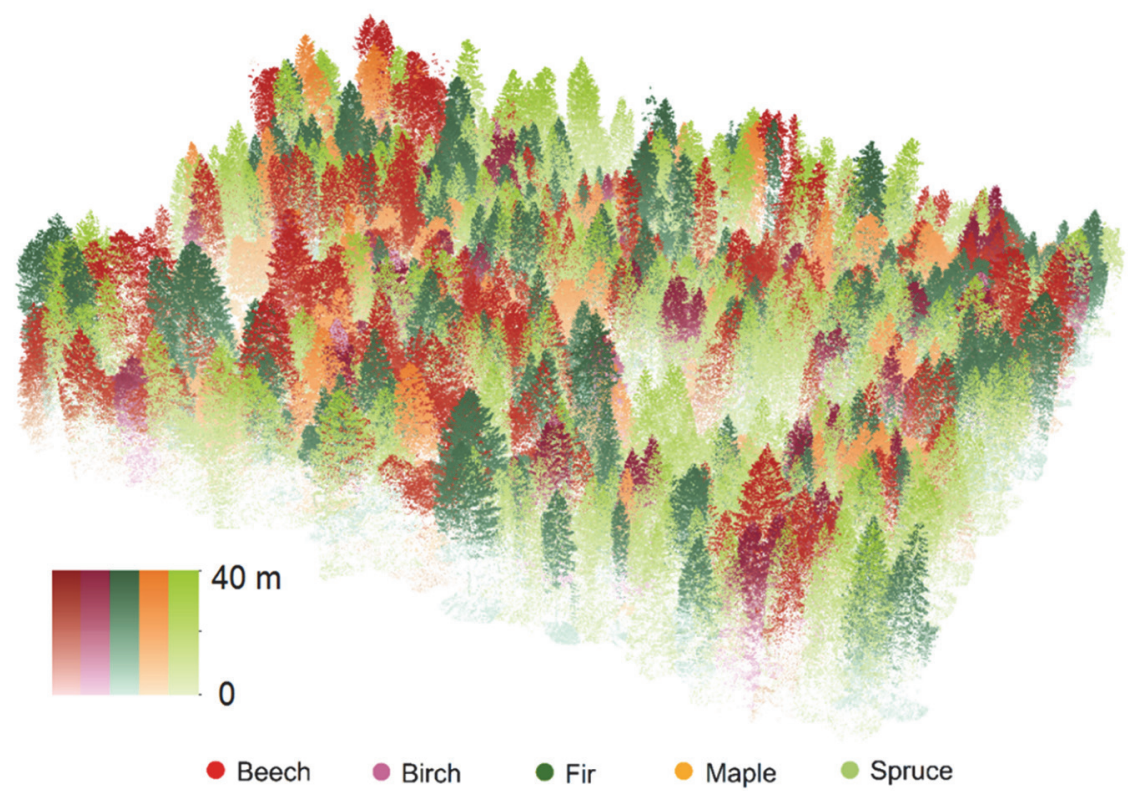

Fig. 4.7 Map of individual tree species classification for an example area (located in site A) in Bavarian Forest National Park.

\subsection{Discussion}

In this study, we integrated three plant functional traits (i.e. $\mathrm{Cw}, \mathrm{Cm}$ and $\mathrm{Cab}$ ) with hyperspectral derived spectral features and airborne LiDAR derived metrics for mapping five tree species in a natural forest. Our results demonstrate that combining retrieved plant functional traits with spectral features and LiDAR metrics yielded the highest classification results, with an overall accuracy of 84.2\% compared to LiDAR (65.1\%) and hyperspectral (69.3\%) variables alone. It is noteworthy that using the retrieved plant functional traits significantly increased tree species classification accuracy - especially when combined with LiDAR metrics. This result validated the assumption that adding characteristic plant functional traits into classification with unique structural profiles aids the discrimination of tree species, which, has a profound meaning for ecology and remote sensing research. The analysis also revealed that using combined datasets (i.e. LiDAR + PFTs, LiDAR + HSI and LiDAR + HSI + PFTs) significantly improved the discrimination of the five tree species compared to using LiDAR or hyperspectral variables alone. Contradictory to the study conducted by Liu et al. (2017), where LiDAR metrics provided greater accuracy than hyperspectral variables, we found that using spectral features from hyperspectral data generated 
higher classification accuracy than using LiDAR metrics. Besides the differences in aimed tree species and employed datasets between these two studies, one reason might be the selection of pixels used for extracting spectral features. In our study, we used only sunlit pixels for spectral information extraction while Liu et al. (2017) used all pixels within a crown. Although there was no significant difference between the classification results using the combination of LiDAR + PFTs and LiDAR + HSI, it should be noted that the slight higher accuracy was obtained using the combination of LiDAR + PFTs with only 15 variables, while LiDAR + HSI involved 27 variables. It implies that combining retrieved plant functional traits with LiDAR metrics for tree species classification can reach a moderate degree of accuracy while avoiding the high data dimensionality which is an inherent characteristic of hyperspectral data.

According to the Random Forest variable importance analysis, equivalent water thickness $(\mathrm{Cw})$ and leaf mass per area $(\mathrm{Cm})$ showed greater importance than most other variables when combined with LiDAR metrics (Fig. 4.5c). When combining retrieved plant functional traits with hyperspectral features and LiDAR metrics, only equivalent water thickness $(\mathrm{Cw})$ consistently performed well among other variables, while leaf mass per area $(\mathrm{Cm})$ and leaf chlorophyll (Cab) achieved similar performances as selected vegetation indices (Fig. 4.5a). Similar performance of selected plant functional traits was observed in previous studies (e.g. Casas et al. 2014; Darvishzadeh et al. 2008; Sehgal et al. 2016). Regardless of the simplification of parameters in RTMs representation and the effect of the forest heterogeneity, it is also argued that there is always poor signal propagation from leaf to canopy level resulting in poor estimation of leaf parameters by canopy reflectance (Asner 1998). The complexity of canopy radiation interaction processes and background effect may also result in misestimating of plant functional traits during the retrievals. While the inversion of RTMs is generally ill-posed because of measurement and model uncertainties, mixed spectra at the level of HySpex pixels also bring additional uncertainties to the retrievals. A promising way to improve the situation of underestimation is taking into account canopy heterogeneity by using 3D RTMs, which could provide an additional advantage in understanding the structural influence upon functional traits retrievals.

LiDAR derived intensity metrics calculated from first-or-single returns played an important role in classifying tree species in this study, which confirmed results found in previous studies (Hovi et al. 2016; Korpela et al. 2010; Shi et al. 2018b). Identified as the most important variables, the mean intensity of first-or-single 
returns not only well represented the structural and morphological characteristics of the outside of the crown, but also reduced multiple scattering effects in discrete return systems. Moreover, the most important spectral features were the first derivation and band reflectance located in $1771.1 \mathrm{~nm}, 1693.2 \mathrm{~nm}$ and $2034.9 \mathrm{~nm}$, related to water content, lignin and cellulose respectively. The important contribution of spectral derivations indicated their potential to enhance pigment absorption features (Blackburn 2007) and to reduce influences of the soil background and other noise (Kokaly and Clark 1999). We also found the anthocyanin content index (ACI2) and shortwave-infrared vegetation index (SWIR_VI) were the two most useful vegetation indices for species discrimination, which also have been supported by Liu et al. (2017), GroßeStoltenberg et al. (2016) and Liu (2016). These vegetation indices were calculated using wavebands located in the green (near $550 \mathrm{~nm}$ ), red (near $650 \mathrm{~nm}$ ) and shortwave infrared (near $2100 \mathrm{~nm}$ and $2210 \mathrm{~nm}$ ). However, spectral variation among species is generated from phenotypic variation and does not directly measure the underlying genetic or phylogenetic relationships among species (Cavender-Bares et al. 2016). Although VIs represent an aggregated measure of significant phenotypic components that influence leaf spectral profiles, plant functional traits retrieved from full spectra allow for a deeper understanding of how tree species differ functionally and may inform our capacity to detect changes in biodiversity.

Our results showed that deciduous trees and coniferous trees were well differentiated by the variables used in this study, however, beech and maple as well as fir and spruce were identified as two pairs of species with similar characteristics and majority misclassifications (Table 4.5 and Fig. 4.7). Given the complexity of natural mixed forests under leaf-on condition, the morphological and spectral similarities among different tree species as well as the architectural variations within the same species might contribute to the misclassification (Shi et al. 2018b). From a morphological perspective, more detailed LiDAR derived structural features, such as branch patterns, may further the distinction between tree species. Meanwhile, from a biological point of view, more precise speciesrelated plant functional traits are valuable to explore for species discrimination. Here, we tested the importance of three plant functional traits retrieved from radiative transfer models, which serves as the substantial markers of tree species that can be used for further study. Although the validation of retrieved plant functional traits in this study was based on the field data with one year difference from the hyperspectral data, Rautiainen et al. (2018) showed that the variation of 
these leaf plant functional traits was minor within species when compared between species during peak season. Still, we suggest the concomitant collection of field data and hyperspectral data collections to minimize uncertainty and potential errors during plant functional traits retrieval. It should be noted that our analysis was based on two study sites with relatively low variability regarding forest type and topography. Therefore, the applicability of the important features needs to be further tested among other tree species in different types of forest.

\subsection{Conclusions}

This study has demonstrated the application of plant functional traits retrieved from RTM in conjunction with airborne LiDAR derived metrics and hyperspectral derived spectral features for individual tree species mapping. Adding plant functional traits to the classification significantly improved the accuracy than using LiDAR or hyperspectral data alone. Equivalent water thickness $(\mathrm{Cw})$ was the most robust plant functional trait for the classification of five tree species in our study, following the mean intensity of first-or-single returns derived from LiDAR data.

Individual tree species classification in temperate forests remains a challenging task. Compared to plantation and urban trees, forest stands are generally denser, deciduous tree crowns are often interlocked, and species mixture is greater and more irregular (Heinzel and Koch 2012). Improving individual tree delineation accuracy from airborne LiDAR data and optimizing feature selection may lead to a higher classification accuracy at individual tree level. Discovering and employing species-specific biophysical and biochemical plant traits remain important to improve the interpretability of both LiDAR and hyperspectral data, with some of the performances revealed here, raising themes for potential further investigations.

Multi-class tree species classification provides an overview of the distribution of tree species while requiring sufficient training data for each class. However, innovative image processing methods for the efficient classification of a focal class, or species, is required. In the next chapter, we successfully map an ecologically important species in the Bavarian Forest National Park using oneclass classification techniques. 


\section{Chapter 5}

\section{Mapping individual silver fir trees in a Norway spruce dominated forest $\$$}

\footnotetext{
$\S$ This chapter is based on: Shi, Y., Wang, T., Skidmore, A.K., Holzwarth, S., Heiden, U., \& Heurich, M.. Individual silver fir (Abies alba) trees accurately mapped using hyperspectral and LiDAR data in a Central European mixed forest. Agricultural and Forest Meteorology. (under review)
} 


\begin{abstract}
Silver fir (Abies alba) is considered an important ecological and functional tree species in European forests. However, in recent centuries, it has experienced a widespread decline across Europe. This study aimed to accurately map individual silver fir trees in a mixed temperate forest in Germany using integrated airborne hyperspectral and LiDAR data. Remote sensing and coincident field data were collected in the study area between 2015 and 2017. A set of spectral and structural features from the hyperspectral and LiDAR data were extracted and used to construct models for testing using different machine learning classification approaches. Specifically, we compared the performance of three one-class classifiers (i.e. one-class support vector machine, biased support vector machine, and maximum entropy) for mapping individual silver fir trees. Our results demonstrated that the biased support vector machine classifier yielded the highest mapping accuracy, with the area under the curve for positive and unlabeled samples ( $p u$ AUC) achieving 0.95 (kappa 0.90). The intensity value of 95th percentile of normalized tree height and the percentage of first returns were found to be the most influential structural features, capturing the main morphological difference between silver fir and Norway spruce at the top tree crown. The wavebands at $700.1 \mathrm{~nm}, 714.5 \mathrm{~nm}$, and $1201.6 \mathrm{~nm}$ were the most important spectral bands, which are strongly affected by chlorophyll and foliar water content. Our study suggests that discovering links between spectral and structural features captured by different remotely sensed data and species-specific traits can improve the accuracy of individual tree species mapping.
\end{abstract}




\subsection{Introduction}

Accurate tree species mapping is essential for a wide variety of applications, including mapping of species composition (Cho et al. 2012; Ørka et al. 2013), invasive species detection (Piiroinen et al. 2018; Somers and Asner 2013a), forest inventories (Bouvier et al. 2015; Yin and Wang 2016), and biodiversity assessment and monitoring (Baldeck et al. 2015; Vaglio Laurin et al. 2014). Large area species distribution maps are also valuable for an improved understanding of the ecology of tree species and the contribution of different species to ecosystem functions and services (Fassnacht et al. 2016; Homolová et al. 2013; Sánchez-Azofeifa et al. 2009).

Silver fir (Abies alba), native to the mountainous regions of Europe, is considered an important ecological and functional balancer of European forests (Tinner et al. 2013). Known as one of the most shade-tolerant European tree species, silver fir has a deep root system, which stabilizes soils and retains water (Lebourgeois et al. 2013), and it is less susceptible to windthrow and snow and ice breakage than Norway spruce (Senn and Suter 2003). Silver fir is a fundamental species for maintaining high biodiversity in forested ecosystems because of its shade tolerance, adaptability to environmental conditions and ability to coexist with many other tree species (Dobrowolska et al. 2017). Silver fir is also an important species in the context of climate change, due to its resistance towards natural disturbances (Desplanque et al. 1999), and it has been shown to respond favourably to climate warming in Central Europe and adjacent areas (Büntgen et al. 2014).

In the second half of the twentieth century, a widespread decline of silver fir has been reported in many mountain regions across Europe (e.g. Elling et al. 2009; Ficko et al. 2011; Vrška et al. 2009). Silver fir is susceptible to a number of abiotic and biotic disturbances, such as late frost, strong winter frost, and browsing by deer (Heuze et al. 2005). It is particularly sensitive to $\mathrm{SO}_{2}$ pollution (Elling et al. 2009), which is one of the reasons for its decline in some regions (Dobrowolska et al. 2017). This decline has been reported to be as high as $80 \%$ in the Carpathians (Vrška et al. 2009) and around 20\% in the Dinaric Mountains (Ficko et al. 2011) in recent decades. Based on the archived inventory documents in the Bavarian Forest National Park (BFNP), the proportion of silver fir in the BFNP was about $60 \%$ in 1856 , while Norway spruce and European beech were only $10 \%$ and $30 \%$, respectively. At the time of the establishment of the BFNP in 1970 , the proportion of fir had decreased to only $3.2 \%$, spruce had increased 
to $72 \%$, while beech remained stable at around $25 \%$ (Heurich and Englmaier 2010). While silver fir had been able to survive the type of forest use practiced by the glassworks, the period of shelter wood and expanding gap management systems led to a near elimination of the species. Both results of pollen analyses and historical descriptions indicate a dramatic decline of fir by at least $90 \%$ in the BFNP over the last two centuries. The increased domination of spruce continued until the late 1990s, when an infestation of bark beetle saw the proportion of fir to spruce increase, along with an increased abundance of other tree species. See Heurich and Englmaier (2010) for a detailed description.

This situation was not unique to the BFNP; similar declines were widespread across Central Europe (Heurich and Englmaier 2010). However, at this point in time, comprehensive maps of silver fir distribution, or indeed methods to accurately and regularly map this important species, are lacking. Silver fir tends to grow as individuals interspersed among other tree species, which makes mapping a particularly challenging task. Traditional plot-level classification methods may not suitable for accurately mapping of silver fir. Furthermore, in Central European mixed forests, silver fir and Norway spruce have been identified as a pair of species with similar spectral and structural characteristics, making it difficult to distinguish between them (Klopčič et al. 2017; Shi et al. 2018a; Vallet and Pérot 2011).

Traditionally, the occurrence of tree species was measured by field sampling, which is accurate but time-consuming and not feasible across large areas. Over the last four decades, advances in remote sensing technologies and machine learning methods have enabled the mapping of tree species from various sensor types (Marrs and Ni-Meister 2019). Hyperspectral sensors and Light Detection and Ranging (LiDAR) system are the most common sources of remotely sensed data used for the classification of tree species (Fassnacht et al. 2016). Hyperspectral sensors measure reflected radiation in hundreds of narrow bands and can detect subtle variations in the biochemical and biophysical properties of the forest canopy (Ferreira et al. 2016; Huber et al. 2008; Somers and Asner 2013b). LiDAR is an active remote sensing technique that uses lasers to capture the three-dimensional structure of forests. Therefore, it is well-suited for individual tree delineation, while also providing valuable geometric and radiometric information for tree species discrimination (Heinzel and Koch 2012; Muss et al. 2011; Vauhkonen et al. 2010a). To capitalize on the datasets from various airborne sensors and their advantages for individual tree species classification, it is necessary to establish connections between spectral and 
structural features derived from remote sensing datasets and the species-specific traits of trees. However, an in-depth understanding of how remotely sensed information depicts the species of trees, in other words, how trees display differently in remote sensing data, is still poorly developed.

A framework for multi-class tree species classification requires that representative training data must be collected for every class, regardless of whether a particular class is of interest to the researcher. In the case of remote tree species mapping, collecting adequate amounts of costly field-based training data for all species in an ecosystem is likely to be intractable (Baldeck and Asner 2015). Consequently, when collected training datasets are not sufficient to adequately characterize every species, understanding of the connection between tree species and remotely senses signatures is limited and the performance of classification is difficult to evaluate. Meanwhile, there is an increasing demand for efficient classification techniques that identify a focal class or species. In this scenario, one-class classification approaches, where labeled data are needed only for the positive class (that is, a single tree species) might be an efficient alternative (Muñoz-Marí et al. 2010). In remote sensing studies, one-class classification approaches have been used to detect focal tree species in tropical rainforests (Baldeck et al. 2015; Somers and Asner 2013b), invasive species detection (Piiroinen et al. 2018; Skowronek et al. 2017), and high nature value grassland habitats (Stenzel et al. 2017). However, the performance of one-class classifiers is highly dependent on the selection of parameters and thresholds (Waske 2017). Further comparison of the discriminative potential of different one-class classifiers - that is, the best achievable performance over all models and thresholds - is still needed for accurate tree species mapping.

The overall objective of this study was to accurately identify and map individual silver fir trees in a spruce-dominated natural forest using airborne LiDAR and hyperspectral data. Specifically, we set out to: (1) generate spectral and structural features for silver fir identification, (2) assess the performance of three one-class classifiers for silver fir mapping, and (3) identify the key spectral and structural features that contributed most to the identification of individual silver fir and understand how they link to species-specific traits. 


\subsection{Materials and Methods}

\subsubsection{Study area and tree species}

Our experiment was carried out in the Bavarian Forest National Park $\left(49^{\circ} 3^{\prime} 19^{\prime \prime}\right.$ $\mathrm{N}, 13^{\circ} 12^{\prime} 9^{\prime \prime} \mathrm{E}$ ), a mixed temperate forest situated in south-eastern Germany (Fig. 5.1). The park covers an area of 24,218 ha with elevations ranging from approximately $600 \mathrm{~m}$ to $1452 \mathrm{~m}$ (Heurich et al. 2010). The mean annual temperature is between $6.5^{\circ} \mathrm{C}$ in the valleys and $2{ }^{\circ} \mathrm{C}$ at higher elevations, and the climate is continental with an annual precipitation varying from 830 to 2230 $\mathrm{mm}$ (Cailleret et al. 2014). The dominant tree species in the national park are Norway spruce (Picea abies) (67\%) and European beech (Fagus sylvatica) (24.5\%), with sliver fir (Abies abies) (2.6\%), sycamore maple (Acer psudoplatanus) (1.2\%), and mountain ash (Sorbus aucuparia) (3.1\%) contributing to the remainder (Heurich and Neufanger 2005).

Within the park, two study sites were selected, each approximately 25 ha $(500 \mathrm{~m}$ $\times 500 \mathrm{~m})$ (Fig. 5.1). Detailed information about the two study sites, including elevation, tree density, soil type and forest type is provided in Table 5.1. The field work was conducted in July 2016 and July 2017, respectively. A Leica Viva GS14 Plus differential GPS (Leica Geosystems AG, Heerbrugg, Switzerland) was used to record the exact location of trees. As a result, 205 locations of trees at site one (T1) and 198 locations of trees at site two (T2) were collected, resulting in 78 beech trees, 58 birch trees, 108 fir trees, 70 maple trees and 89 spruce trees. The collected GPS data was post-processed to obtain differentially corrected coordinates with an accuracy less than $0.25 \mathrm{~m}$. 


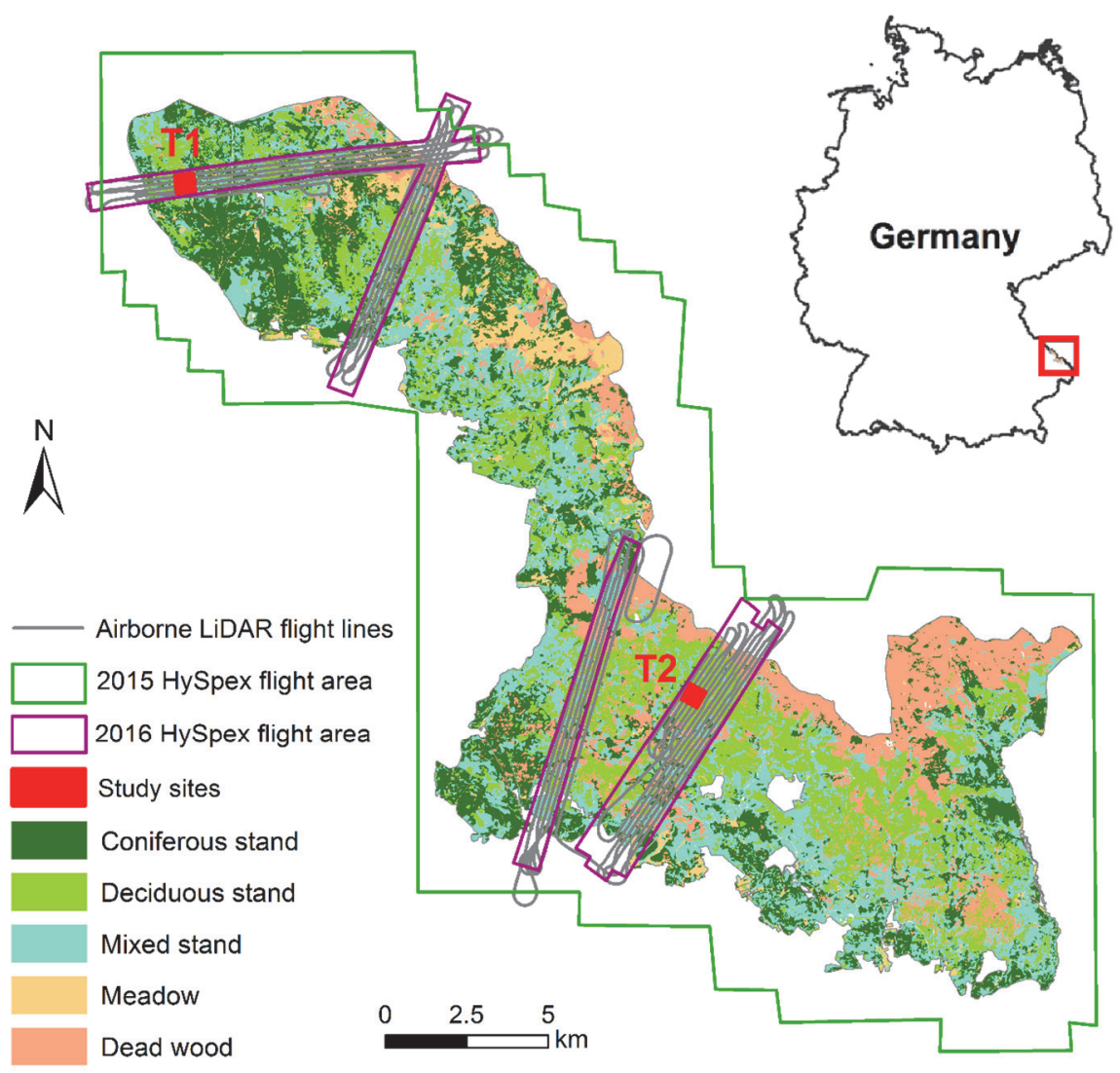

Fig. 5.1 Airborne LiDAR and HySpex flight area and the location of two study sites in the Bavarian Forest National Park, Germany.

Table 5.1 Characteristics of the two study sites

\begin{tabular}{llllll}
\hline $\begin{array}{l}\text { Study } \\
\text { sites }\end{array}$ & $\begin{array}{l}\text { Size } \\
\text { (ha) }\end{array}$ & $\begin{array}{l}\text { Elevation } \\
(\mathrm{m})\end{array}$ & $\begin{array}{l}\text { Tree } \\
\text { density } \\
\text { (per ha) }\end{array}$ & Soil type & Forest type \\
\hline & & & & $\begin{array}{l}\text { Brown forest } \\
\text { soils and peat } \\
\text { soils }\end{array}$ & $\begin{array}{l}\text { Mature } \\
\text { coniferous and } \\
\text { mixed stands }\end{array}$ \\
T2 & 25 & $675-732$ & 445 & $\begin{array}{l}\text { Loose brown } \\
\text { soils and gley } \\
\text { soils }\end{array}$ & $\begin{array}{l}\text { Mature } \\
\text { deciduous and } \\
\text { mixed stands }\end{array}$ \\
\hline
\end{tabular}




\subsubsection{Data acquisition and pre-processing}

\subsubsection{Airborne LiDAR data}

The airborne LiDAR data was collected by Milan Flug GmbH on 18 August 2016, covering four transects in the Bavarian Forest National Park (Fig. 5.1). LiDAR data processing and preparation was described in Chapter 2, section 2.2.4.

\subsubsection{Multi-temporal hyperspectral data}

Two hyperspectral flight campaigns were carried out by DLR (German Aerospace Centre) with the same HySpex sensor system, developed by Norsk Elektro Optikk (NEO), on 26 August 2015 and 25 August 2016, respectively. The HySpex sensor consists of two imaging spectrometers covering spectral ranges of 400-992 nm (VNIR) and 968-2498 nm (SWIR), with spectral resolutions of $3.6 \mathrm{~nm}$ and $6 \mathrm{~nm}$, respectively. The acquisition time, flight altitude, spectral and spatial resolution for each dataset are displayed in Table 5.2. The HySpex datasets were supplied by DLR after the unified pre-processing procedures. The preprocessing procedures included the following steps: radiance conversion and system correction using laboratory radiometric calibration information (Gege et al. 2009); atmospheric correction performed with the ATCOR4 model (Richter and Schläpfer 2002); ortho-rectification of the radiance data based on the parametric model and flight path data in combination with a digital terrain model (DEM) (Müller et al. 2005) and co-registration of VNIR and SWIR data cubes using brisk and sensor-model-based RANSAC (Schwind et al. 2014). A Savitzky-Golay filter was applied to correct for random and systematic noise (Schläpfer and Richter 2011). After eliminating the bands affected by strong noise or atmospheric effects (water vapour absorption), 290 bands for each dataset remained. Approximately 40 ground control points of each study site were chosen for the co-registration of hyperspectral and LiDAR data using a polynomial warp method ( 2 degree) and nearest neighbour resampling method in the ENVI software (version 5.2). The resulting geometric accuracy was higher than $0.20 \mathrm{~m}$. 
Table 5.2 The parameters of HySpex datasets

\begin{tabular}{lll}
\hline Acquisition date & 26 August 2015 & 25 August 2016 \\
\hline Acquisition time (UTC) & $10: 24$ & $08: 51$ \\
Flight altitude (m. asl) & 3544 & 2331 \\
Spectral resolution VINR (nm) & 3.6 & 3.6 \\
Spectral resolution SWIR (nm) & 6 & 6 \\
Spatial resolution VNIR (m) & 2 & 1 \\
Spatial resolution SWIR (m) & 4 & 2 \\
\hline
\end{tabular}

\subsubsection{Segmentation of individual trees}

The individual tree segmentation was described in Chapter 2, see section 2.2.3. We visually verified the identified sample trees that were visible in the two hyperspectral images using the procedure proposed in section 2.2.3. In total, 90 fir trees, 77 beech trees, 56 birch trees, 68 maple trees and 88 spruce trees from the two study sites were selected for further analysis. Once the sample trees were verified, the 3D points within each correct segment were extracted and assigned to the corresponding sample trees for the derivation of LiDAR metrics.
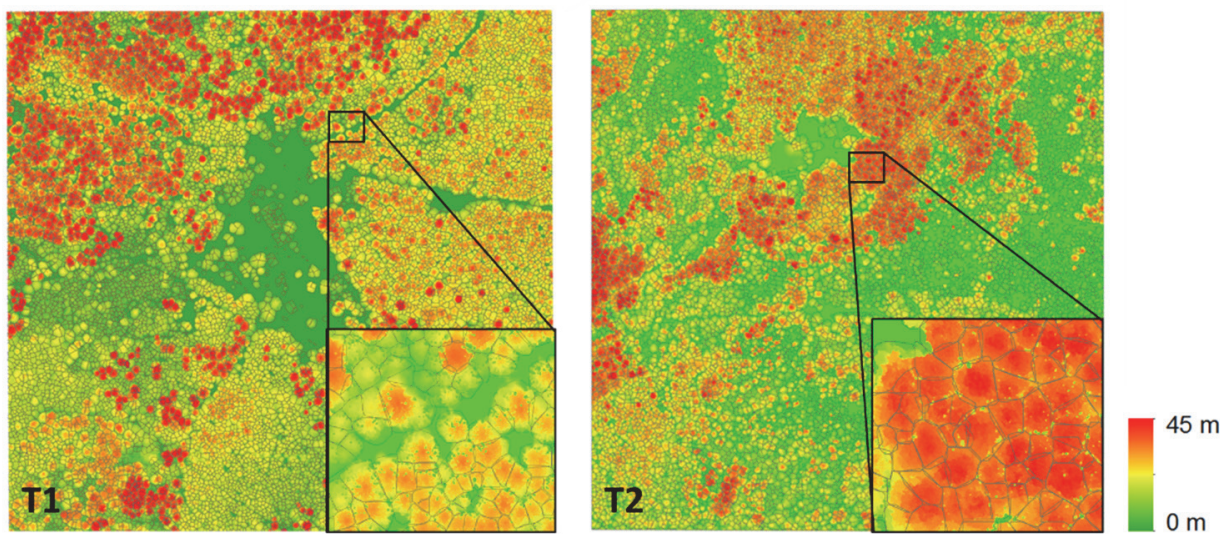

Fig. 5.2 Segmented individual tree crowns for the two study site.

\subsubsection{Feature generation}

Previous studies demonstrated that sunlit pixels of hyperspectral images can often provide more accurate species information for classification (e.g. Clark et al. 2005; Dalponte et al. 2013; Richter et al. 2016). To reduce the effect of shadowing, as well as the errors in individual tree crown delineation, we manually 
selected the sunlit pixels within each tree crown and extracted the spectral information. The mean spectral value of each waveband (B1, B2, .., B290) from each year's hyperspectral data was calculated within each tree crown. Based on the results of Chapter 4, we also derived four vegetation indices: the Anthocyanin Content Index (ACI2), the Disease-Water Stress Index (DWSI2), the Red-edge Vegetation Stress Index (RVSI), and the Shortwave-Infrared Vegetation Index (SWIR_VI), which is related to plant pigment content, water content and stress (Table 5.3).

To better understand how LiDAR metrics represent the structural characteristics of tree species, we classified the LiDAR metrics into three primary categories (Table 5.3). Specifically, these included (1) point distributions, which reflect the structural features of different tree species with the number of laser points tend to decrease from tree top to bottom due to laser obstruction by crowns (Lin and Hyyppä 2016); (2) radiometric metrics (i.e. intensity and echo width), which suggest that laser amplitudes tend to deteriorate from tree top to bottom, and have different behaviours according to the foliage type, leaf size and density from different tree species; (3) geometric metrics, including tree height, crown shape and crown volume features. The derivation of LiDAR metrics was conducted using the " $r$ LiDAR" package in the R language environment (http://www.rproject.org/). 
Table 5.3 List of generated LiDAR metrics and hyperspectral features

\begin{tabular}{|c|c|c|c|}
\hline Category & Generated features & Definition or formula & Reference \\
\hline \multirow[t]{8}{*}{$\begin{array}{l}\text { Point } \\
\text { distributions }\end{array}$} & Percent_first & Percentage of first returns above $2 \mathrm{~m}$ & $\begin{array}{l}\text { (Puttonen et al. } \\
2010)\end{array}$ \\
\hline & Percent_last & Percentage of last returns above $2 \mathrm{~m}$ & $\begin{array}{l}\text { (Dalponte et al. } \\
\text { 2012) }\end{array}$ \\
\hline & Percent_all & Percentage of all returns above $2 \mathrm{~m}$ & (Korpela et al. 2010) \\
\hline & Percent_first_mean & Percentage of first returns above mean height & \\
\hline & Percent_all_mean & Percentage of all returns above mean height & \\
\hline & All_counts & Total counts of all returns & \\
\hline & First_counts & Total counts of first returns & \\
\hline & $\begin{array}{l}\text { Counts_returns (1st- } \\
7 \text { th) }\end{array}$ & $\begin{array}{l}\text { The counts of different number of returns ( } 1 \text { st } \\
-7 \text { th returns) }\end{array}$ & \\
\hline \multirow[t]{4}{*}{$\begin{array}{l}\text { Radiometric } \\
\text { metrics }\end{array}$} & $\begin{array}{l}\text { Imean, Isd, Ivar, Icv, } \\
\text { Ikur, } \\
\text { Iske, Ip_nth }\end{array}$ & $\begin{array}{l}\text { Intensity parameters in the tree crown, } \\
\text { including mean value (Imean), standard } \\
\text { deviation (Isd), variation (Ivar), coefficient } \\
\text { variance (Icv), kurtosis (Ikur), skewness } \\
\text { (Iske), and } n \text {th percentile of intensity (5th, } \\
10 \text { th, ..., 95th, 99th) }\end{array}$ & $\begin{array}{l}\text { (Dalponte } \text { et al. } \\
\text { 2008) } \\
\text { (Yao et al. 2012) } \\
\text { (Heinzel and Koch } \\
\text { 2011) }\end{array}$ \\
\hline & $\begin{array}{l}\text { Imean_single, } \\
\text { Imean_first }\end{array}$ & $\begin{array}{l}\text { The mean intensity value of single returns } \\
\text { (Imean_single), } \\
\text { the mean intensity value of the first returns } \\
\text { (Imean_first) }\end{array}$ & \\
\hline & $\begin{array}{l}\text { EWmean, EWsd, } \\
\text { EWvar, EWcv, EWkur, } \\
\text { EWske, EWp_nth }\end{array}$ & $\begin{array}{l}\text { Echo width parameters in the tree crown, } \\
\text { including mean value (EWmean), standard } \\
\text { deviation (EWsd), variation (EWvar), } \\
\text { coefficient variance (EWcv), kurtosis } \\
\text { (EWkur), skewness (EWske), and } n \text {th } \\
\text { percentile of echo width (5th, 10th, ..., 95th, } \\
\text { 99th) }\end{array}$ & \\
\hline & $\begin{array}{l}\text { EWmean_single, } \\
\text { EWmean_first }\end{array}$ & $\begin{array}{l}\text { The mean echo width of single returns } \\
\text { (EWmean_single), } \\
\text { the mean echo width of the first returns } \\
\text { (EWmean_first) }\end{array}$ & \\
\hline \multirow[t]{3}{*}{$\begin{array}{l}\text { Geometric } \\
\text { metrics }\end{array}$} & $\begin{array}{l}\text { Height, Hmean, Hsd, } \\
\text { Hvar, Hcv, Hkur, Hske, } \\
\text { Hp_nth }\end{array}$ & $\begin{array}{l}\text { Height parameters of the tree, including tree } \\
\text { height (Height), mean value (Hmean), } \\
\text { standard deviation (Hsd), variation (Hvar), } \\
\text { coefficient variance (Hcv), kurtosis (Hkur), } \\
\text { skewness (Hske), and } n \text {th percentile of height } \\
\text { (5th, 10th, .., 95th, 99th) }\end{array}$ & $\begin{array}{l}\text { (Li } \text { et al. 2013) } \\
\text { (Lin and Herold } \\
2016 \text { ) } \\
\text { (Puttonen } \text { et al. } \\
\text { 2010) }\end{array}$ \\
\hline & $\begin{array}{l}\text { Hmean_single, } \\
\text { Hmean_first }\end{array}$ & $\begin{array}{l}\text { The mean height of single returns } \\
\text { (Hmean_single), } \\
\text { the mean height of first returns (Hmean_first) }\end{array}$ & \\
\hline & $\begin{array}{l}\text { CBH:H, C_volume:area, } \\
\text { CNR }\end{array}$ & $\begin{array}{l}\text { Ratio of crown base height to height }(\mathrm{CBH}: \mathrm{H}) \text {, } \\
\text { ratio of crown volume to crown area } \\
\text { (C_volume:area), and canopy relief ratio } \\
\text { (CNR) }\end{array}$ & \\
\hline $\begin{array}{l}\text { Band } \\
\text { reflectance }\end{array}$ & B1-B290 & Band reflectance from 415.7-2496.5 nm & \\
\hline \multirow[t]{4}{*}{$\begin{array}{l}\text { Vegetation } \\
\text { indices }\end{array}$} & $\mathrm{ACI} 2$ & $\begin{array}{l}\text { Anthocyanin Content Index, } \\
\text { ACI } 2=\rho 650 / \rho 550\end{array}$ & $\begin{array}{l}\text { (Gamon and Surfus } \\
1999 \text { ) }\end{array}$ \\
\hline & DWSI2 & $\begin{array}{l}\text { Disease-Water Stress Index, } \\
\text { DWSI2 }=\rho 1660 / \rho 550\end{array}$ & (Apan et al. 2004) \\
\hline & RVSI & $\begin{array}{l}\text { Red-edge Vegetation Stress Index, } \\
\text { RVSI }=(\rho 714+\rho 752) / 2-\rho 733\end{array}$ & (Merton 1998) \\
\hline & SWIR_VI & $\begin{array}{l}\text { Shortwave-Infrared Vegetation Index, } \\
\text { SWIR_VI }=37.72 *(\rho 2210-\rho 2090) \\
+26.27 *(\rho 2280-\rho 2090)+0.57\end{array}$ & (Lobell et al. 2001) \\
\hline
\end{tabular}




\subsubsection{Feature selection approaches}

Feature selection is a procedure that enables a meaningful interpretation of the selected predictors and, in the context of tree species classification, increases the understanding of what exactly drives the discrimination of the species (Fassnacht et al. 2016). To select the most valuable wavebands from the hyperspectral images, we aimed to optimize the spectral separability between fir and other species, and used the principles of the Uncorrelated Stable Zone Unmixing approach proposed by Somers and Asner (2013b) for spectral bands selection. The rationale for this method is to balance the relationship between the spectral separability and the spectral correlation in the final subset (Somers and Asner 2013b). Firstly, the spectral separability between fir and the other species (i.e. fir and beech, fir and birch, fir and maple, and fir and spruce) was evaluated using the Separability Index (SI), defined as the ratio of the inter-species and the intraspecies variability:

$$
S I_{i}=\frac{\Delta_{\text {inter }, i}}{\Delta_{\text {intra }, i}}=\frac{\left|R_{\text {mean }, 1, i}-R_{\text {mean }, 2, i}\right|}{1.96 \times\left(\sigma_{1, i}+\sigma_{2, i}\right)}
$$

where $R_{\text {mean }, 1, i}$ and $R_{\text {mean }, 2, i}$ are the mean reflectance values at wavelength $i$ for species 1 (i.e. fir) and species 2 (e.g. beech, birch, maple, and spruce), respectively, whereas $\sigma_{1, i}$ and $\sigma_{2, i}$ are the standard deviations of species 1 and 2, respectively. Higher SI values indicate greater separability between the species in the specified waveband (Somers and Asner 2013b). Secondly, the spectral correlation (Corr) of the selected band with all the other wavebands was calculated according to:

$$
\operatorname{Corr}(X, Y)=\frac{\operatorname{cov}(X, Y)}{\sigma_{X} \sigma_{Y}}
$$

where $\operatorname{cov}(X, Y)$ is the covariance between the selected band $(X)$ and the other wavebands $(Y)$, and $\sigma$ is the standard deviation of the wavebands. Finally, the selection of wavebands was done iteratively by repeatedly selecting the band with the highest separability index and removing the highest correlated band until no bands remained (Somers and Asner 2014).

To select the final set of features (i.e. from both the hyperspectral and LiDAR metrics) we employed a wrapper algorithm using Support Vector Machines (SVM), a method proposed by Maldonado and Weber (2009). It is based on a sequential backward selection, which uses the number of errors in a validation 
subset as the measure to decide which feature to remove in each iteration. This approach has several advantages with respect to the objectives of the current study, including (1) it determines the contribution of each feature to the respective classifier, (2) it is capable of measuring the validation error while avoiding overfitting by doing a random split of the dataset in each iteration, and (3) it can be easily generalized to variations of SVM classifiers (Maldonado and Weber 2009). The feature selection procedure was carried out with the packages "caret" and "e1071" in the R language environment (http://www.r-project.org/).

\subsubsection{One-class classifiers}

Among various one-class classifiers, one-class support vector machine (OCSVM), biased support vector machine (BSVM) and Maxent have been frequently used (Mack and Waske 2017). The OCSVM (Schölkopf et al. 1999) uses only data from the class of interest to train the classifier, while the BSVM is a semi-supervised classification algorithm that utilizes both positive and unlabelled samples (Liu et al. 2003). The BSVM is a special form of a binary SVM and is adapted to one-class classification with a positive and unlabelled data training set (Stenzel et al. 2017). The Maxent classifier is based on the maximum entropy approach (Sethna 2006), which is able to perform efficiently even with few occurrence records (Pearson 2007).

We used only positive and unlabelled data ( $P U$-data) during the model training. The spectral features derived from each hyperspectral image, along with the LiDAR metrics, were used in three different one-class modelling approaches, tuned with optimal parameters for fir classification (Table 5.4). More information on the kernel parameters, the method and the criteria can be found in the description of the R package "oneClass" (https://github.com/benmack/oneClass) and the corresponding publication (Mack et al. 2014). 
Mapping individual silver fir trees in a Norway spruce dominated forest

Table 5.4 Description of one-class classifiers

\begin{tabular}{llll}
\hline $\begin{array}{l}\text { One-class } \\
\text { classifiers }\end{array}$ & $\begin{array}{l}\text { Training } \\
\text { mode }\end{array}$ & Parameters & Tuning settings \\
\hline OCSVM & $P$-classifier & $\begin{array}{l}\text { Sigma }(\sigma) \text { : the width of Gaussian } \\
\text { radial basis function (RBF) kernel }\end{array}$ & $2^{-10}, 2^{-9}, \ldots, 2^{2}$ \\
& & $\mathrm{Nu}(v)$ : rejection fraction & $0.01,0.02, \ldots, 0.5$ \\
BSVM & $P U$-classifier & $\begin{array}{l}\text { Sigma }(\sigma) \text { : the width of Gaussian } \\
\text { radial basis function (RBF) kernel }\end{array}$ & $2^{-4}, 2^{-3}, \ldots, 2^{2}$ \\
& & $\begin{array}{l}\text { cNeg: penalty parameter for } \\
\text { unlabeled samples }\end{array}$ & $2^{-7}, 2^{-5}, \ldots, 2^{2}$ \\
& & $\begin{array}{l}\text { cMultiplier: penalty parameter for } \\
\text { positive samples }\end{array}$ & $2^{0}, 2^{1}, \ldots, 2^{6}$ \\
Maxent & $P U$-classifier & $\begin{array}{l}\text { Fc: feature class (lineal }(\mathrm{L}), \\
\text { quadratic }(\mathrm{Q}), \text { product }(\mathrm{P}),\end{array}$ & $\mathrm{LQHPT}$ \\
& & threshold (T), and hinge (H)) & \\
& & Beta $(\beta)$ : regularization multiplier & $1,2, \ldots, 40$ \\
\hline
\end{tabular}

\subsubsection{Mapping accuracy assessment}

The classifiers were trained using a 10 -fold cross-validation strategy. The training samples were split into 10 independent sets (folds), in which 9 were used for training and the remaining one for validation. After repeating 10 times, the best combination of parameters was chosen by minimizing an average error measurement computed with the predictions on the 10 different validation sets (Muñoz-Marí et al. 2010). We selected the best performing model based on the ranking of puAUC (Phillips et al. 2006; Phillips and Dudík 2008), which resembles the area under the receiver operator characteristic curve (AUC) as an independent measure using randomly sampled observations (Piiroinen et al. 2018). The Kappa coefficient was also measured for the each model. In comparative studies, it is rare to see Kappa reported, due to lack of absence samples in the validation data. However, it remains informative since it reveals the relative accuracy of the $P U$ data-based model selection approaches (Waske 2017). 


\subsection{Results}

\subsubsection{Differences in spectral and structure features between silver fir and other four tree species}

Fig. 5.3 shows the mean spectral signatures (400-2498 nm) of five tree species, derived from the hyperspectral data of 2015 and 2016. The spectral signature of fir is clearly distinguishable from the deciduous trees (i.e. beech, birch, and maple), but the difference between fir and spruce is minor. The difference between the spectral signatures of fir and spruce was larger in the 2016 data than that of 2015 .

Fig. 5.4 shows the four vegetation indices (ACI2, DWSI2, RVSI and SWIR_VI), derived from 2015 and 2016, for the five species. The differences between conifers and deciduous trees were more distinct in RVSI and SWIR_VI than in ACI2 and DWSI2. The variation pattern of vegetation indices among the five species was similar in 2015 and 2016, while for each single species, the distribution of vegetation indices varied between 2015 and 2016.

Fig. 5.5 shows the variation of derived LiDAR metrics between fir and the other four tree species. Six metrics from each LiDAR category (i.e. "Percent_first" and "Percent_all" from point distributions, "Ip95" and "EWmean_single" from radiometric metrics and "Hp99" and "Hmean_first" from geometric metrics) are displayed as examples. Fig. 5.5a shows that the variation of "Percent_first" (i.e. the percentage of first returns above $2 \mathrm{~m}$ ) of fir is the smallest among the five species. Fig. 5.5c and Fig. 5.5f show distinct differences for the 99th percentile of tree height (Hp99) and the mean height of first returns (Hmean_first) between fir and deciduous trees. However, the differences between fir and spruce were minor. 

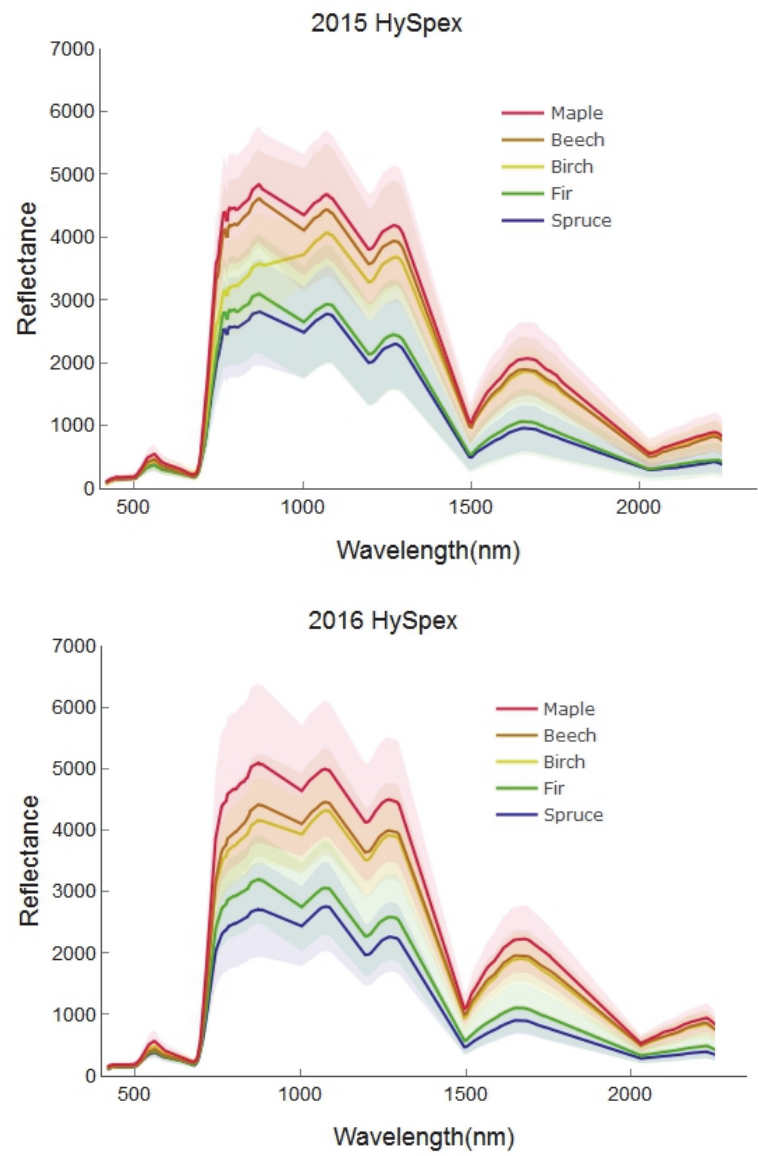

Fig. 5.3 The mean reflectance value $(\times 1000, \pm 1$ standard deviation) of five species (i.e. beech, birch, fir, maple and spruce) at 400-2498 nm wavelengths derived from HySpex data acquired in 2015 and 2016, respectively. 

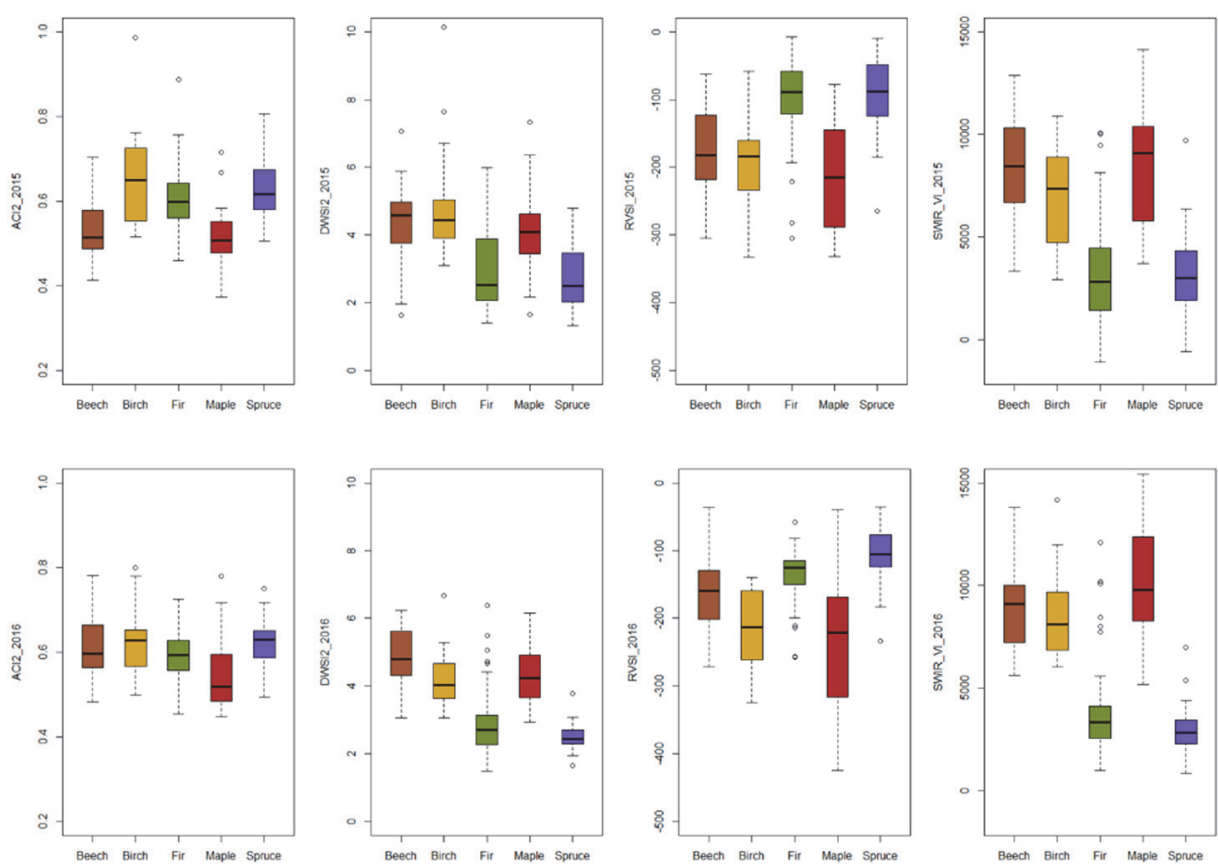

Fig. 5.4 Box plots of four vegetation indices (i.e. ACI2, DWSI2, RVSI and SWIR_VI) derived from 2015 (first row) and 2016 (second row) among sample tree species. 

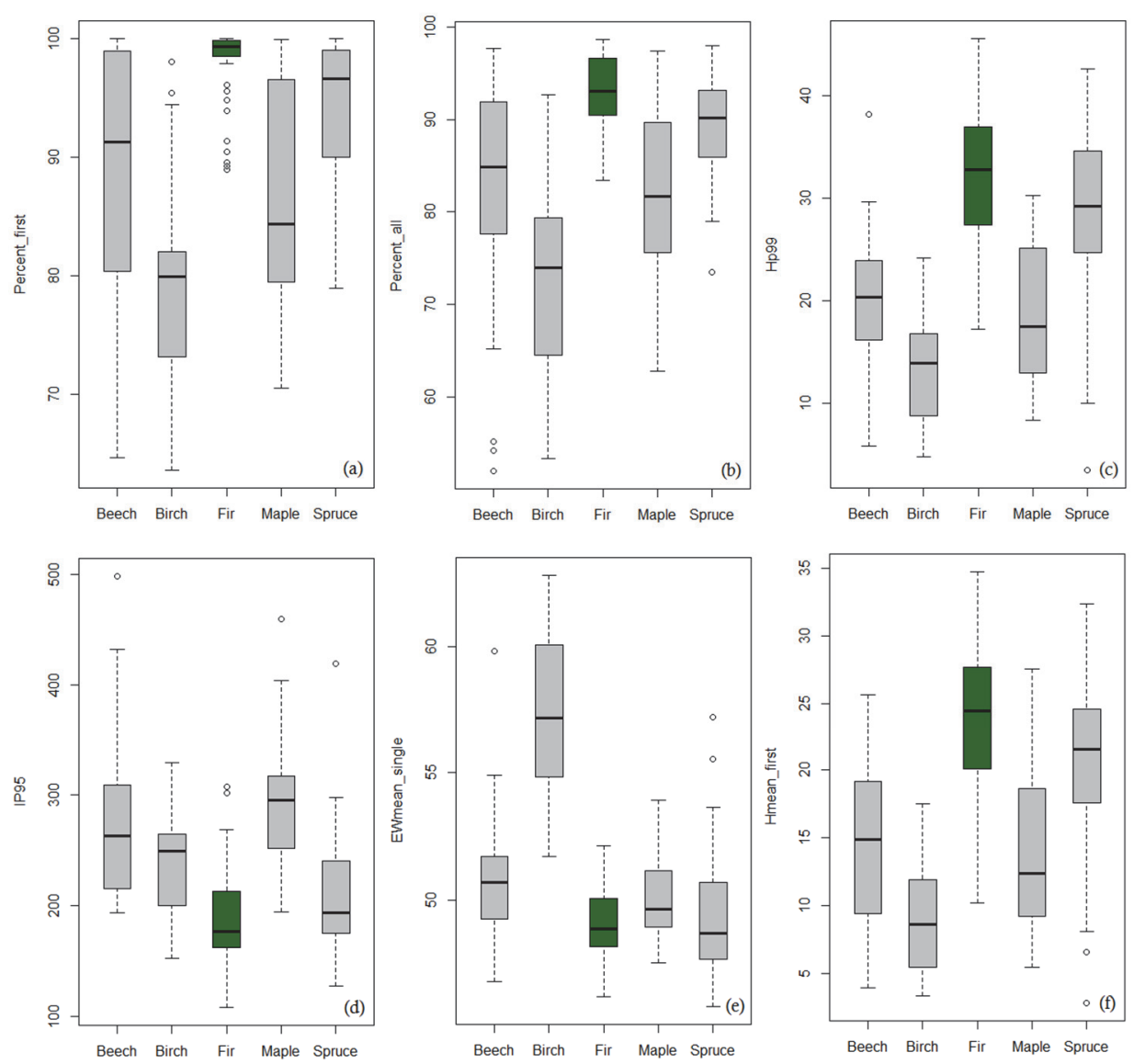

Fig. 5.5 Box plots of the percentage of first returns above $2 \mathrm{~m}$ (a), the percentage of all returns above $2 \mathrm{~m}$ (b), 99 th percentile of tree height (c), the intensity of 95 th percentile of normalized tree height $(\mathrm{d})$, the mean echo width of single returns (e), the mean height of first returns (f) among sample tree species.

\subsubsection{Feature selection results}

Based on the spectral separability and correlation assessment, we selected a set of bands maximizing the separability between each combination of two species. Fig. 5.6 shows the spectral separability index (SI) between fir and the other four tree species (i.e. maple, beech, birch, and spruce) based on each year of HySpex data. The most distinguishable wavelengths between all pairs were located at 689.3-743.3 nm, 1087.7-1219.6 nm, and 2244.7-2412.6 nm. After feature selection, using the Uncorrelated Stable Zone Unmixing approach and the wrapper SVM algorithm (see section 2.5), 18 wavebands from the 2015 HySpex, 
19 wavebands from the 2016 HySpex, five vegetation indices calculated from both years, and 14 LiDAR metrics, were selected for fir classification (Table 5.5).
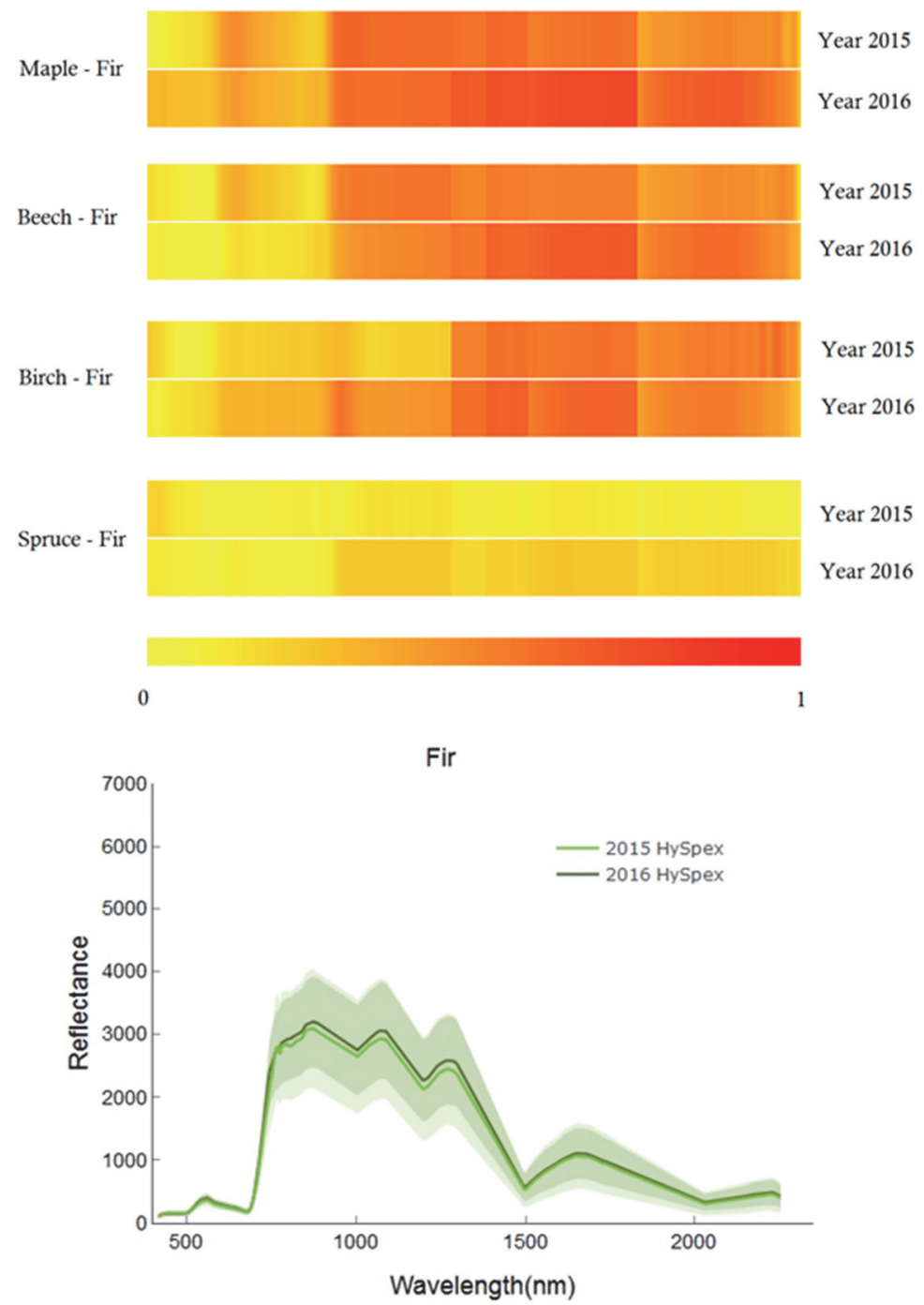

Fig. 5.6 The spectral separability index (SI) between fir and other four tree species (i.e. maple, beech, birch, and spruce) in the year of 2015 and 2016. 0 indicates the lowest SI and 1 indicates the highest SI between two species. 
Mapping individual silver fir trees in a Norway spruce dominated forest

Table 5.5 Selected features derived from hyperspectral and LiDAR data

\begin{tabular}{|c|c|}
\hline Input variables & Index or description \\
\hline \multicolumn{2}{|l|}{ LiDAR metrics } \\
\hline Imean_first & Mean intensity of first-or-single returns \\
\hline Imean_single & Mean intensity of single returns \\
\hline Imean & Mean intensity \\
\hline Ivar & Variation of intensity \\
\hline Isd & Standard deviation of intensity \\
\hline Ip95 & Intensity of 95th percentile of normalized tree height \\
\hline Ewmean_single & Mean echo width of single returns \\
\hline EWp55 & $\begin{array}{l}\text { Echo width of } 55 \text { th percentile of normalized tree } \\
\text { height }\end{array}$ \\
\hline Hmean_first & Mean height of first-or-single returns \\
\hline Hmean_single & Mean height of single returns \\
\hline Hvar & Variation of height \\
\hline Hp99 & 99th percentile of normalized tree height \\
\hline Percent_first & Percentage of first returns above $2 \mathrm{~m}$ \\
\hline Percent_all & Percentage of all returns above $2 \mathrm{~m}$ \\
\hline \multicolumn{2}{|c|}{ Spectral features (HSI) } \\
\hline \multicolumn{2}{|c|}{ Band reflectance (nm) } \\
\hline 2015 HySpex & $\begin{array}{l}415.7 \mathrm{~nm}, 433.7 \mathrm{~nm}, 502.1 \mathrm{~nm}, 527.3 \mathrm{~nm}, 692.9 \mathrm{~nm} \text {, } \\
700.1 \mathrm{~nm}, 714.5 \mathrm{~nm}, 732.5 \mathrm{~nm}, 764.9 \mathrm{~nm}, 872.9 \mathrm{~nm} \text {, } \\
1201.6 \mathrm{~nm}, 1219.6 \mathrm{~nm}, 1597.3 \mathrm{~nm}, 2106.8 \mathrm{~nm}, \\
2262.7 \mathrm{~nm}, 2382.6 \mathrm{~nm}, 2400.6 \mathrm{~nm}, 2418.6 \mathrm{~nm}\end{array}$ \\
\hline 2016 HySpex & $\begin{array}{l}419.3 \mathrm{~nm}, 437.3 \mathrm{~nm}, 458.9 \mathrm{~nm}, 678.5 \mathrm{~nm}, 689.3 \mathrm{~nm} \text {, } \\
692.9 \mathrm{~nm}, 700.1 \mathrm{~nm}, 707.3 \mathrm{~nm}, 714.5 \mathrm{~nm}, 746.9 \mathrm{~nm} \text {, } \\
854.9 \mathrm{~nm}, 883.7 \mathrm{~nm}, 1195.6 \mathrm{~nm}, 1591.3 \mathrm{~nm}, 1723.2 \\
\mathrm{~nm}, 1771.1 \mathrm{~nm}, 2070.9 \mathrm{~nm}, 2406.6 \mathrm{~nm}, 2412.6 \mathrm{~nm}\end{array}$ \\
\hline \multicolumn{2}{|c|}{ Vegetation Indices } \\
\hline $\mathrm{ACI} 2$ & $\mathrm{ACI} 2=\rho 650 / \rho 550$ \\
\hline SWIR_VI & $\begin{array}{l}\text { SWIR_VI=37.72*( }(\rho 2210-\rho 2090)+26.27 *(\rho 2280- \\
\rho 2090)+0.57\end{array}$ \\
\hline DWSI2 & DWSI $2=\rho 1660 / \rho 550$ \\
\hline RVSI & $\mathrm{RVSI}=(\rho 714+\rho 752) / 2-\rho 733$ \\
\hline
\end{tabular}

\subsubsection{Performance of classification}

Table 5.6 shows the one-class classification results of silver fir using three different classifiers. Classifications using BSVM generated higher accuracy than OCSVM and Maxent. The classification result using BSVM with LiDAR and HySpex data from 2016 achieved the highest accuracy ( $p u$ AUC 0.95 and Kappa 0.90), while the model using 2015 HySpex data had the same puAUC and a slightly lower Kappa value (0.89). 
Table 5.7 shows the significant levels between classification results generated from different combinations based on $p$ values in McNemar's test. Significant improvements were found between the results from BSVM and the results using OCSVM or Maxent.

Fig. 5.7 shows the normalized importance of selected features from hyperspectral and LiDAR data for fir classification. The most important spectral bands for fir classification varied between the different HySpex acquisitions: for 2015, the most important bands were $700.1 \mathrm{~nm}, 714.5 \mathrm{~nm}, 1201.6 \mathrm{~nm}, 1219.6 \mathrm{~nm}, 2262.7$ $\mathrm{nm}$, and $2382.6 \mathrm{~nm}$, while for 2016, the most important bands were $700.1 \mathrm{~nm}$, $714.5 \mathrm{~nm}, 1201.6 \mathrm{~nm}, 1591.3 \mathrm{~nm}, 1723.2 \mathrm{~nm}, 1771.1 \mathrm{~nm}, 2070.9 \mathrm{~nm}$. However, there were several bands that were considered important in both years $(700.1 \mathrm{~nm}$, $714.5 \mathrm{~nm}$, and $1201.6 \mathrm{~nm}$ ) (Fig. 5.7). The percentage of first returns above $2 \mathrm{~m}$ (Percent_first) and the intensity of 95th percentile of normalized tree height (Ip95) were the most important LiDAR metrics for fir classification.

Fig. 5.8 displays the fir mapping results using 2016 HySpex and LiDAR data, based on the optimized BSVM model, for the two study sites. The mapped fir crowns are marked in yellow against the background of a colour-infrared aerial photograph. The point clouds of fir trees are highlighted in red.

Table 5.6 One-class classification results of fir trees from hyperspectral and LiDAR data using three different classifiers

\begin{tabular}{lcccc}
\hline & \multicolumn{2}{c}{ LiDAR+2015 HySpex } & \multicolumn{2}{c}{ LiDAR+2016 HySpex } \\
& $p u$ AUC & Kappa & $p u$ AUC & Kappa \\
\hline OCSVM & 0.89 & 0.87 & 0.90 & 0.87 \\
BSVM & 0.95 & 0.89 & 0.95 & 0.90 \\
Maxent & 0.83 & 0.82 & 0.87 & 0.85 \\
\hline
\end{tabular}

Table 5.7 McNemar's test for pairwise comparison between classification results using different classifiers. NS: $p>0.05 . * * * p<0.001{ }^{* *} p<0.01 .{ }^{*} p<0.05$.

\begin{tabular}{lllll} 
& \multicolumn{2}{l}{ LiDAR +2015 HySpex } & \multicolumn{2}{l}{ LiDAR +2016 HySpex } \\
& OCSVM & Maxent & OCSVM & Maxent \\
\hline BSVM & $*$ & $* *$ & $*$ & $*$ \\
Maxent & $*$ & - & NS & - \\
\hline
\end{tabular}



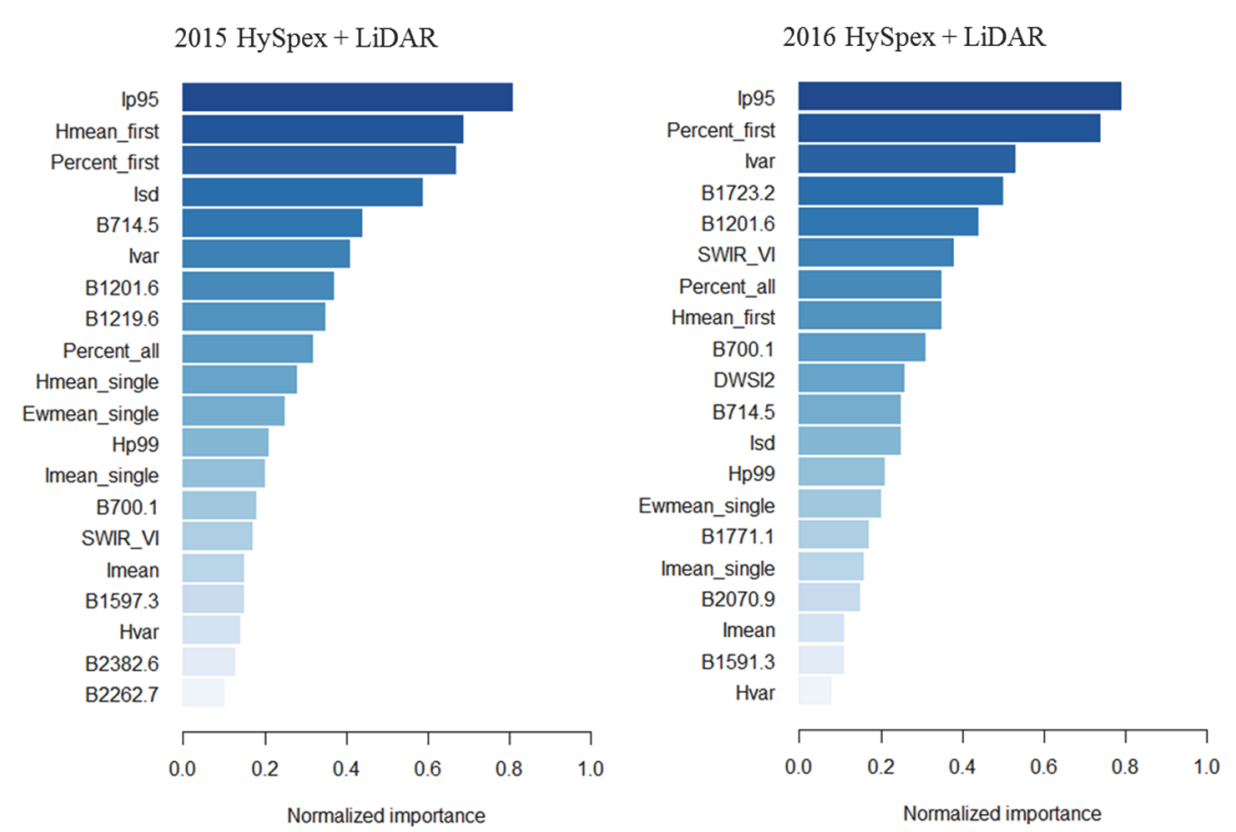

Fig. 5.7 The normalized importance of selected features from the combination of each year HySpex and LiDAR data for fir classification.
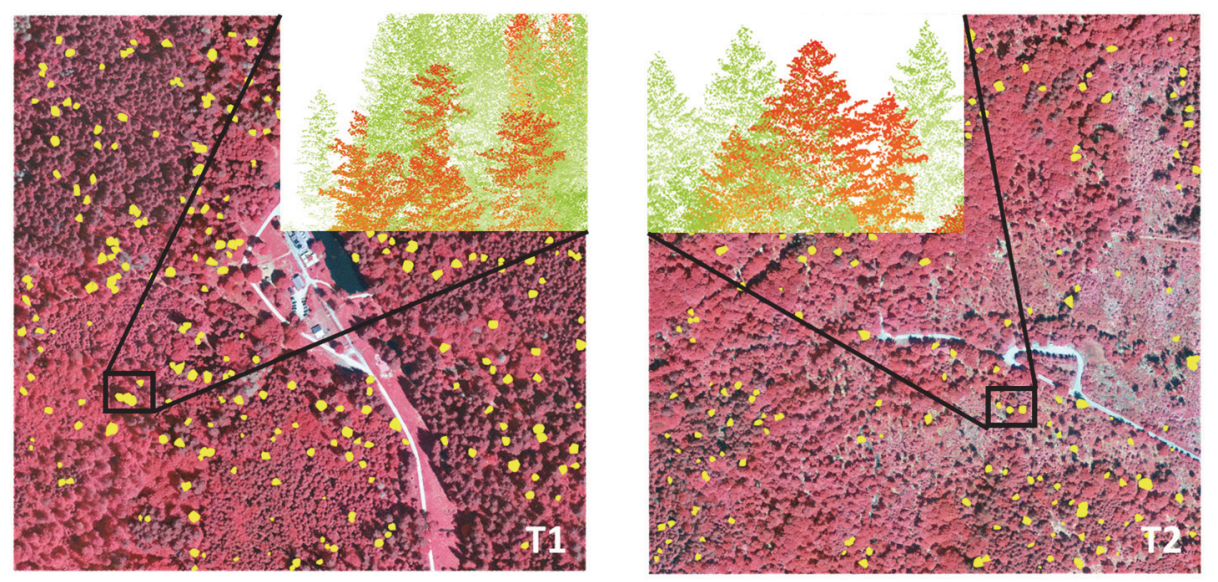

Fig. 5.8 Maps of fir trees in two study sites $(500 \mathrm{~m} \times 500 \mathrm{~m}$ for each site $)$ in the Bavarian Forest National Park. The crown of fir trees are highlighted in yellow. The point clouds of mapped fir are highlighted in red.

\subsection{Discussion}

This study accurately mapped individual silver fir trees in a Norway spruce dominated forest using one-class classification methods, employing key spectral 
and structure features closely linked to species-specific traits. Our results demonstrated that the biased support vector machine classifier yielded the highest mapping accuracy, with the area under the curve for positive and unlabeled samples (puAUC) achieving 0.95 (kappa 0.90).

Identifying key features that can reflect the specific traits of tree species is an important issue for tree species classification. Our study revealed that the most robust spectral bands from HySpex datasets for mapping of silver fir were located at wavebands $700.1 \mathrm{~nm}$ and $714.5 \mathrm{~nm}$, which are strongly affected by leaf chlorophylls (Ustin et al. 2009), as well as the waveband of $1201.6 \mathrm{~nm}$, which is sensitive to foliar water content (Kokaly et al. 2009). This result is in line with Gitelson et al. (2003) and Ustin et al. (2009), who indicated that the total chlorophyll content in leaves is closely related to the green $(540-560 \mathrm{~nm})$ and red edge (700-730 nm) wavelengths. In Chapter 4, leaf chlorophyll (Cab) and equivalent water thickness $(\mathrm{Cw})$ were measured from field samples of five tree species (i.e. beech, birch, fir, maple and spruce); fir showed the highest leaf chlorophyll (mean: $56.3 \mu \mathrm{g} \mathrm{cm}^{-2}$, sd: $11.0 \mu \mathrm{g} \mathrm{cm}^{-2}$ ) and equivalent water thickness (mean: $0.0166 \mathrm{~cm}$; sd: $0.0012 \mathrm{~cm}$ ) among the five tree species. Norway spruce, as the other conifer, caused the main confusion in fir classification. However, it showed a comparatively low leaf chlorophyll (mean: $34.5 \mu \mathrm{g} \mathrm{cm}{ }^{-2}$, sd: $6.4 \mu \mathrm{g} \mathrm{cm}$ ${ }^{2}$ ) and equivalent water thickness (mean: $0.0140 \mathrm{~cm}$; sd: $0.0024 \mathrm{~cm}$ ). This highlights that optimizing spectral separability between the focal tree species and others can help the discrimination of tree species, since absorption features caused by biochemical composition control the shapes of leaf reflectance spectra. It is worth noting that the specific wavebands that contribute most in a focal species mapping are highly depend on the species of interest and the neighboring species (e.g. the species with similar reflectance) as well as the forest characteristics (e.g. homogeneity and heterogeneity).

Our results indicated that the most important LiDAR metrics for fir identification were the percentage of first returns (Percent_first) and the intensity of 95th percentile of normalized tree height (Ip95). The percentage of first returns reflects the shape of "crown shell" and the pattern of outer layers of a tree. Similarly, the intensity of 95th percentile of normalized tree height is related to the distribution of branches at the top of the canopy. From an autecological point of view, silver fir has a pyramidal crown that becomes flat-topped with age - the so called stork's nest - while spruce displays a conic crown with an ascending upper level and drooping lower level (Farjon 1990; Silba 1986) (Fig. 5.9). The variation of the "Percent_first" captured the "flat-topped" crown traits of fir, which was the 
smallest among other tree species. Our results suggest that the structural differences at the top of canopy among tree species that can be captured by airborne LiDAR are valuable for tree species discrimination. Moreover, combining the complimentary information from both airborne hyperspectral and LiDAR data provides additional perspectives for the discrimination of the focal species.

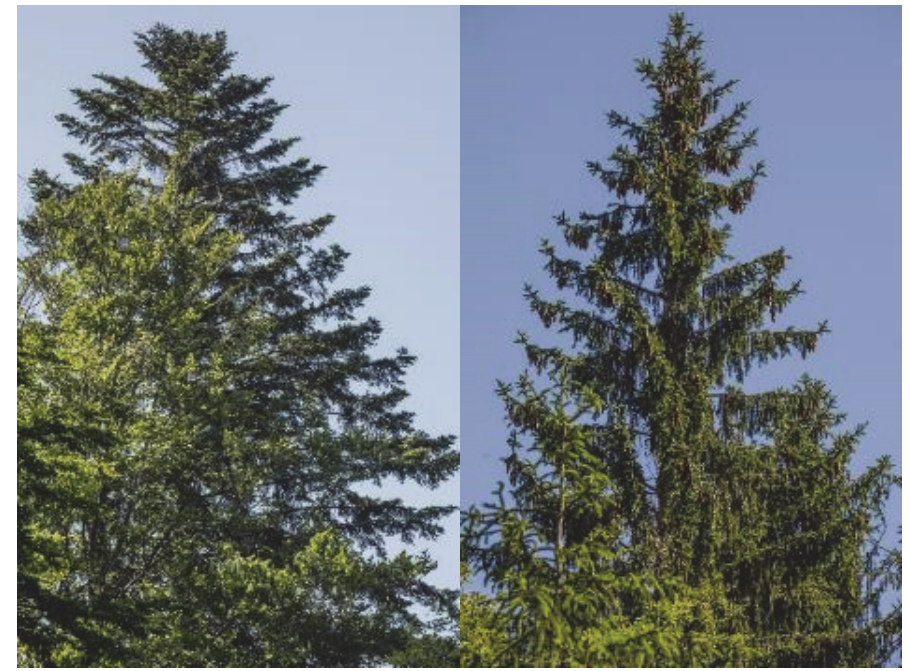

(a) Sliver fir

(b) Norway spruce

Fig. 5.9 The crown shape of a silver fir tree (a) and a Norway spruce tree (b). (Photos by Rainer Simonis)

We did not find a significant difference between the classification results using HySpex at $4 \mathrm{~m}$ (2015) and HySpex at $2 \mathrm{~m}$ (2016) (0.03-0.06 improvement of puAUC and 0.02-0.04 improvement of Kappa). The slight difference between the results may due to the variances in illumination conditions, the number of reference pixels as well as the spatial resolution from different years. Given that the crown size of the sample trees in this study were 5-12 m, a spatial resolution smaller than the scale of tree crowns was found to be adequate for individual tree species discrimination, as also indicated by Clark et al. (2005). Our reason for using two HySpex images in this study was to evaluate the robustness of the selected features and classification methods. Given the similar acquisition date of the year and the focal species (i.e. fir as a conifer species), we did not attempt to capture phenological changes among different years for classification. However, it is notable that our study was situated in a natural temperate forest in Central 
Europe, the optimal spatial resolution for individual tree species mapping in other forests is closely related to the ecosystem under consideration.

In this study, the BSVM classifier produced the highest mapping accuracies under all dataset combinations, especially in comparison to Maxent. Similar results have been reported in previous studies. For example, Waske (2017) showed that BSVM had the highest discriminative potential, followed by Maxent (with parameter tuning), Maxent (with default parameters) and OCSVM, while Stenzel et al. (2017) found that BSVM outperformed Maxent (with default parameters) and OCSVM in the classification of high nature value grassland areas (Piiroinen et al. 2018). Although the model performance has been optimized by searching for the best combination of parameters in our study, it should be noted that input feature combinations may also influence the performance of classifiers, as mentioned by Skowronek et al. (2017). This suggests that the correlation between input features as well as the optimization of parameters within the models should be both considered for improving classification results.

Our study accurately mapped silver fir in a natural temperate forest using oneclass classification, however, broadly speaking, tree species mapping via remote sensing remains challenging. The mapping accuracy of the focal species is highly dependent on the tree species diversity in the study area, the similarity between the species of interest and co-existing species, and the amount of field data available. More presence and absence field data may be required before the model can be applied over a larger extent. Furthermore, as mentioned by Budei et al. (2018), having a diversity of tree ages in the sampled crowns comparatively increases the intra-species variability and the classification error probability because of the changes in tree architecture, leaf shapes and reflectance with tree age. Given the architectural variability of the top crown of fir between different ages, for example, an understanding of whether stratification by tree age or environmental factors (e.g. soil type and topographic condition) could improve classification performance should be explored in future research.

\subsection{Conclusions}

This study demonstrated that spectral and structure features derived from airborne hyperspectral and LiDAR data can capture species-specific traits, which, when used in a one-class classification model, can accurately identify the ecologically important silver fir trees in a temperate mixed forest. The mapping results identified areas with a high occurrence probability of silver fir trees, which can increase the efficiency of subsequent field campaigns for forest management and 
biodiversity monitoring. The methodology we demonstrated in this study could be applied in the mapping of other tree species, which enriches the knowledge of species-specific characteristics and the related remotely sensed signatures. Forest managers and policy makers increasingly require individual tree species information, which can inform management strategies to assist forest inventory in larger spatial scales and protect important species such as the silver fir. 


\section{Chapter 6}

Synthesis: Mapping individual tree species using multi-source remotely sensed data 


\subsection{Summary}

Accurately identifying individual tree species is crucial for economic and ecological applications. Tree species mapping is also important for forestry production and conservation management. Therefore, more efficient techniques for mapping of tree species are required (Lin and Hyyppä 2016).

This thesis explored species-specific features derived from various remote sensing datasets, including digital aerial colour-infrared photographs, airborne hyperspectral and LiDAR data for accurately mapping individual tree species using machine learning algorithms. Chapter 2 evaluated the performance of 37 frequently used LiDAR metrics derived under leaf-on and leaf-off conditions, respectively, for discriminating six tree species in a mixed natural forest. It revealed that radiometric features, especially intensity related metrics, provided more consistent and significant contributions than geometric features for tree species discrimination. Chapter 3 examined whether multi-temporal colourinfrared (CIR) orthophotos can be used to improve airborne LiDAR-based individual tree species mapping in a natural forest. The results showed that texture features derived from multi-temporal CIR orthophotos (three years) under different illumination conditions can capture the species-specific differences which significantly improved the species classification performance. Chapter 4 integrated three plant functional traits (i.e. equivalent water thickness, leaf mass per area and leaf chlorophyll) retrieved from hyperspectral data with hyperspectral derived spectral features and airborne LiDAR derived metrics for tree species mapping. We found that adding plant functional traits significantly improved the classification accuracy than using LiDAR or hyperspectral data alone. Chapter 5 chose silver fir (Abies alba) as an example focal species in the Bavarian Forest National Park and identified key spectral and structure features derived from remotely sensed data closely linked to species-specific traits, which contributed accurately mapping of this species using one-class classification. Morphological and spectral difference between silver fir and other four tree species were identified and linked with species-specific traits, leading higher mapping accuracy of silver fir.

The region covered in Chapters 2, 3, 4 and 5 comprised two study sites identified in the Bavarian Forest National Park. The fieldwork was conducted in 2016 and 2017. However, due to the different datasets employed in each chapter and the amount of sample trees coinciding with the integrated datasets, the sample size of tree species differs from each chapter. Moreover, although the Random Forest 
algorithm was employed in Chapters 2, 3 and 4, it serves different purposes in the thesis through evaluating various integrated features derived from multi-source remotely sensed data.

\subsection{The potential of geometric and radiometric features derived from LiDAR in tree species mapping}

The availability of LiDAR system to measure three-dimensional information of trees, such as foliage distributions and branching patterns, provides an opportunity to significantly improve forest tree species classification accuracy ( $\mathrm{Li}$ et al. 2013). Among the LiDAR derived metrics, the geometric metrics describe the geometric structure of trees (e.g. crown shape, tree height and crown volume) while the radiometric metrics refer to specific echo parameters that are extracted from the received waveform (e.g. the backscatter cross-section, the energy of laser points, and the distance between two waveform echoes) (Koenig and Höfle 2016; Wagner 2010). Over the past decade, a large number of airborne LiDARderived metrics have been proposed for tree species classification (Kim et al. 2011; Ørka et al. 2009). Yet an in-depth ecological and biological understanding of the significance of these metrics for tree species mapping remains largely unexplored.

The potential of LiDAR derived metrics is essential for efficiently and accurately mapping individual tree species. Chapter 2 explored the potential of frequently used geometric and radiometric LiDAR metrics under leaf-on and leaf-off conditions for the classification of six tree species in a mixed temperate forest. Chapter 2 indicated that radiometric features consistently contributed a higher accuracy compared to geometric features for classifying tree species under both leaf-on and leaf-off conditions. Particularly, the mean intensity of first-or-single returns provides consistent valuable contribution for tree species discrimination due to its ability of depicting the outer "crown shell" of trees. It implies that the difference in the spatial distribution of branches between different species is most prominent at the top of canopy. However, many geometric features are affected by tree height, which may be also related to other properties, such as crown volume, crown shape and the interior structure of the tree crown. Misclassifications may be due to the similarities of the morphology among different tree species and also the architectural variations within the same tree species. For future research, more detailed radiometric features derived from multi-spectral LiDAR related to internal foliage and branch patterns of an individual tree may be used to increase species discrimination. 


\subsection{The contribution of multi-temporal airborne remotely sensed data in tree species mapping}

An important contribution of remotely sensed data to tree species mapping is its potential to capture the changes that occur in different tree species over extended period of time. Multi-temporal passive remote sensing data, such as aerial photographs, multispectral and hyperspectral imagery, can capture the spectral signatures linked to biochemical and biophysical attributes of tree species during different phenological periods (e.g. bud burst, leaf flushing, flowering, senescence and dormancy) (Fang et al. 2018). Multi-temporal active remote sensing data, such as LiDAR under leaf-on and leaf-off conditions, can measure the changes of three dimensional features describing species differences in crown architecture and structure (Fig. 6.1). Digital colour-infrared (CIR) aerial photographs, which have been collected routinely in many parts of the world, are an invaluable data source for the monitoring and assessment of forest resources. Yet, the potential of these data for automated mapping of individual tree species remains largely unexplored. Moreover, due to the incompatibility of hyperspectral and LiDAR collections, data availability as well as the high costs associated with acquisitions and data processing efforts, the full potential of multi-temporal remotely sensed datasets for tree species classification has yet to be realized.

To evaluate the capacity of multi-temporal LiDAR data for discriminating tree species, chapter 2 tested 37 frequently used LiDAR metrics under leaf-on and leaf-off conditions to classify six different tree species in the Bavarian Forest National Park. Chapter 2 indicated a significant improvement of overall accuracy and kappa value when combining leaf-on and leaf-off LiDAR metrics (OA: 66.5\%, kappa: 0.58) than using only leaf-on LiDAR metrics (OA: 58.2\%, kappa: 0.47) or only leaf-off LiDAR metrics (OA: 62.0\%, kappa: 0.51). However, there was no statistically significant difference between the use of leaf-on and leaf-off LiDAR metrics. Nonetheless, due to the LiDAR metrics under leaf-off condition may offer a better chance to describe the interior structure of trees, a large proportion of important metrics were generated under leaf-off condition (Shi et al. 2018b). 


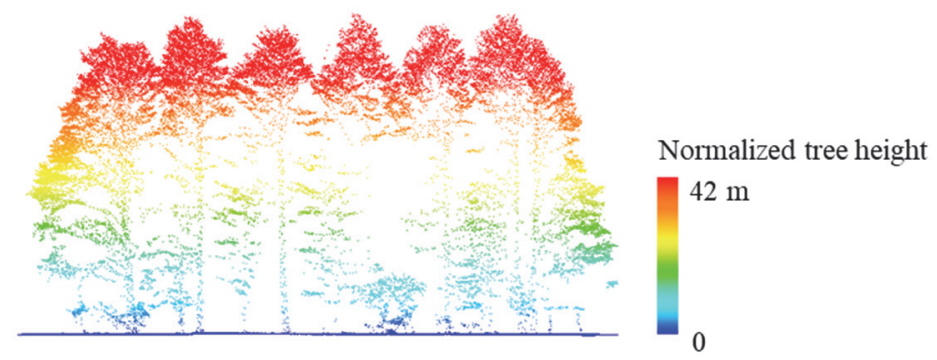

Leaf-off
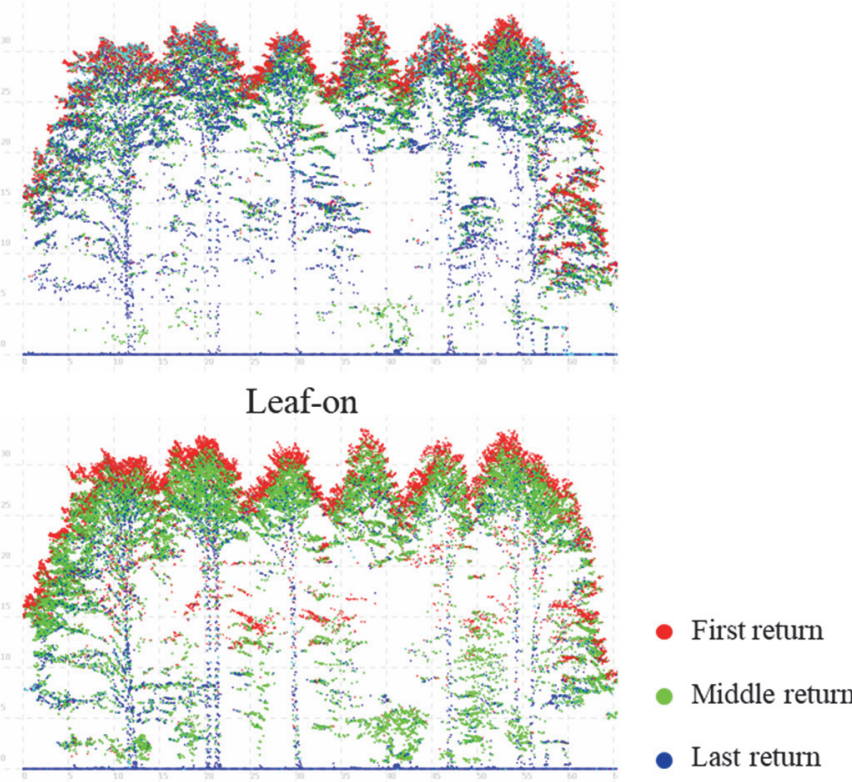

Fig. 6.1 An example of the LiDAR returns under leaf-on and leaf-off conditions

To examine the role of multi-temporal aerial photographs in LiDAR-based individual tree species mapping, chapter 3 demonstrated how texture features generated from multi-temporal digital CIR orthophotos under different viewillumination conditions can further improve the mapping accuracy in a temperate mixed forest. Species-specific information can be captured by the texture features derived from multi-temporal digital aerial photographs with different illumination geometry. Due to the high correlation between texture features (especially those derived from the same year) as well as the spectral variance within same species, only combining digital CIR orthophotos from more than two years with LiDAR data delivered a statistical significant improvement compared with using LiDAR data alone. 
To explore the potential of multi-temporal hyperspectral data for individual tree species mapping, spectral, spatial and temporal variability are important considerations for classifying tree species (Dudley et al. 2015). Chapter 5 presented an example of how hyperspectral imageries with different spatial resolution ( $2 \mathrm{~m}$ from 2016 and $4 \mathrm{~m}$ from 2015) and one-year time difference can be used for a focal tree species mapping in conjunction with LiDAR data. The difference between the classification results using $2 \mathrm{~m}$ and $4 \mathrm{~m}$ hyperspectral imageries was marginal. However, the multi-temporal hyperspectral data were mostly collected in July and August in the Bavarian Forest National Park, thus, phenological changes can hardly see from the datasets. Further research is required to understand how multi-temporal hyperspectral imageries may capture distinct phenological periods for tree species mapping.

\subsection{The role of remotely sensed plant functional traits in tree species mapping}

The characteristics of different tree species closely related to genetics, environmental conditions and the biophysical and biochemical compositions (Kozhoridze et al. 2016). Due to the improvements in 400-2500 nm imaging spectrometers, the capabilities of remote sensing to determine plant functional traits have been established during the past few decades (Asner and Martin 2009; Féret and Asner 2011; Martin et al. 2018). Previous studies have indicated that tree species vary markedly in leaf water content (Huber et al. 2008), leaf mass per area (Casas et al. 2014), nitrogen and carbon concentration (Huber et al. 2008; Wang et al. 2016). For example, based on the data collected in a highly diverse tropical forest, Asner and Martin (2009) found that spectral signatures linked to seven leaf functional traits (chlorophyll a, chlorophyll b, carotenoids, nitrogen, phosphorus, water content, and specific leaf area) are unique for 41 tree species. Based on our fieldwork conducted in July 2017, six tree species (including 53 beech trees, 37 birch trees, 44 fir trees, 44 maple trees, 12 rowan trees, 47 spruce trees) show distinct differences of measured plant functional traits (Fig 6.2). 

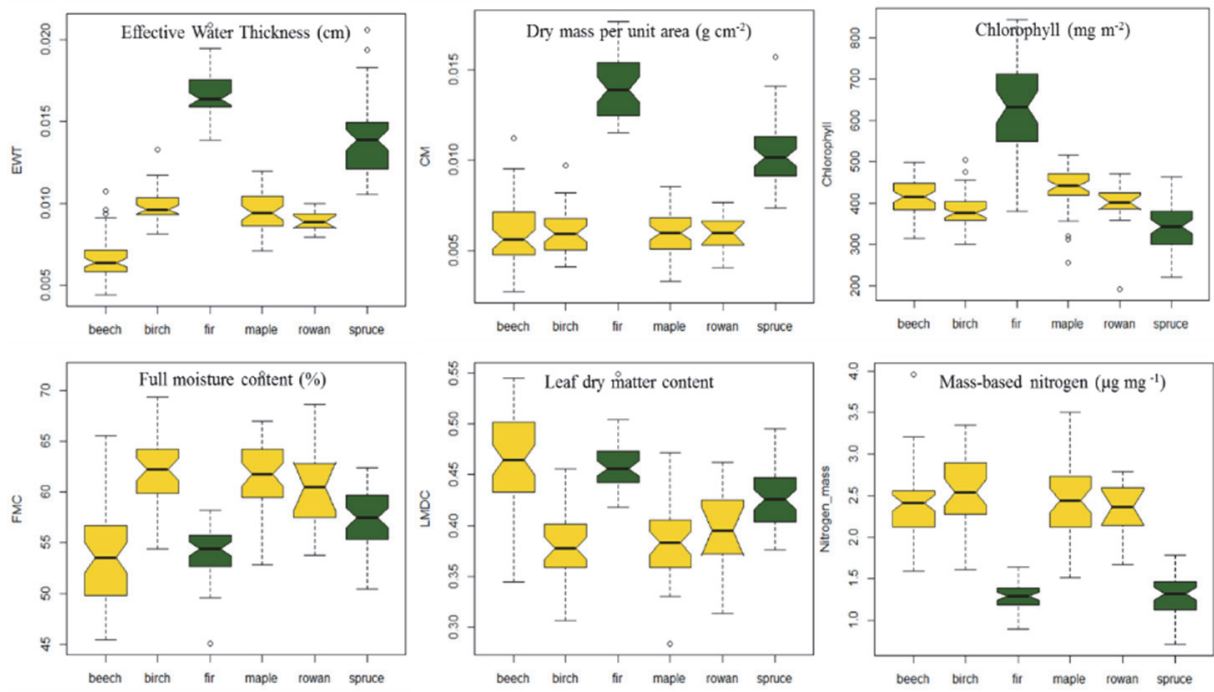

Fig. 6.2 Plant functional traits measured in the field among six tree species in the Bavarian Forest National Park, Germany.

Plant functional traits have been extensively used to describe, rank and discriminate species according to their variability between species in classical plant taxonomy. However, the utility of plant functional traits for tree species classification from remotely sensed data in natural forests has not been clearly established. In chapter 4, we have hypothesized that combining plant functional traits retrieved from hyperspectral data with airborne LiDAR-derived metrics and spectral features generated from hyperspectral data could improve the performance of tree species classification. One of the radiative transfer models (RTMs) - INFORM model was used to retrieve three plant functional traits (i.e. equivalent water thickness $(\mathrm{Cw})$, leaf mass per area $(\mathrm{Cm})$ and leaf chlorophyll (Cab)). Chapter 4 demonstrated that when plant functional traits (i.e. equivalent water thickness $(\mathrm{Cw})$, leaf mass per area $(\mathrm{Cm})$ and leaf chlorophyll $(\mathrm{Cab}))$ were combined with spectral features and LiDAR metrics, an overall accuracy of $84.2 \%$ was obtained, which was statistically significantly higher than using LiDAR (65.1\%) or hyperspectral $(69.3 \%)$ data alone. Using the retrieved plant functional traits significantly increased tree species classification accuracy - especially when combined with LiDAR metrics. This result validated the assumption that adding species-specific plant functional traits into classification with unique structural profiles aids the discrimination of tree species, which, has a profound meaning for ecology and remote sensing research. 
To further explore the potential of the various types of remote sensing data for applications dedicated to the accurate retrieval of plant functional traits of tree species, realistic and accurate simulations of forest ecosystems are required (Ferreira et al. 2018). More comprehensive 3D RTMs, such as the discrete anisotropic radiative transfer (DART) model, can be employed to simulate the radiative transfer of forest canopies, helping to interpret the radiometric signal measured by remote sensing. Combining airborne hyperspectral, LiDAR and in situ measurements for 3D RTMs parameterizing and plant functional traits retrieving for tree species classification purposes need to be further explored in the future work.

\subsection{Improving mapping accuracy of single tree species by connecting remotely sensed features and species- specific traits}

From an ecological and biological perspective, classification of individual tree species requires species-specific features, which can be morphological (e.g. tree height, tree branching pattern), physiological (e.g. photosynthesis rate), biochemical (e.g. leaf chlorophyll, leaf water content) or phenological (e.g. leaf phenology, flowering time) features (Shi et al. 2018a). Tree species mapping with remote sensing should be linked to an understanding that tree species have unique structural profiles and spectral signatures as well as genetic characteristic properties (Asner et al. 2009; Cho et al. 2010; Clark et al. 2005). Given the advantage of various remote sensing sensors, discovering the connections between spectral and structural features derived from remote sensing datasets and species-specific traits of trees becomes crucial for accurate classification of individual tree species. However, an in-depth understanding of how remotely sensed information depicts the species of trees, in other words, how trees display differently in remote sensing data, is still poorly developed. Moreover, the critical obstacles in traditional multi-class tree species classification remain challenging, such as the limited samples size, inter-species similarities, intra-species variations, feature redundancy and classifiers inapplicability.

The principle of tree species classification is to identify species-specific traits and link them to remotely sensed signals. Chapter 5 used silver fir as the focal tree species, and demonstrated a workflow of accurately mapping this species using species-specific traits. Chapter 5 showed that multi-source remote sensing datasets (i.e. LiDAR and hyperspectral data) can provide complimentary speciesspecific information. For example, wavebands of $700.1 \mathrm{~nm}, 707.3 \mathrm{~nm}$ from 
HySpex data provide great spectral information to identify fir trees, which are strongly affected by chlorophylls of leaves. Meanwhile, the best performed LiDAR metrics - the percentage of first returns and the 95th percentile of intensity capture the main morphological difference between silver fir and Norway spruce which appears at the top of tree crown. Given the architectural variability of top crown of fir between different age as an example, the understanding of whether stratification by tree age or environmental factors (e.g. soil type and topographic condition) could improve classification performance should be explored in future research.

\subsection{The role of machine learning techniques in tree species mapping}

Machine learning, a family of statistical techniques with origins in the field of artificial intelligence, is recognized as holding great promise for efficient processing of remote sensing data and modeling ecological systems (Olden et al. 2008). For remotely sensing the species of individual trees, machine learning has the capacity of handling datasets with high dimensionality, reduce variable redundancy and noise, and simplify the process of combining datasets (Marrs and Ni-Meister 2019). Many previous studies have attempted to identify and map individual tree species based on the developments in remote sensing technology coupled with machine learning techniques (e.g. Dalponte et al. 2019; Kamal and Phinn 2011; Lim et al. 2019; Marrs and Ni-Meister 2019; Maxwell et al. 2018). It is also increasingly easy to implement such techniques: free, user-friendly data mining and machine learning applications have already been made available in software such as Matlab, R, and Python.

Historically, visual interpretation of aerial photography was the most popular form of remote sensing of forests (Barrett et al. 2016; Spurr 1960). Compared to this traditional means based on expert knowledge, machine learning techniques allow the computer to learn from input data and establish a workflow presenting the prediction. The identification of spectral, textural, and structural variables from various remotely sensed datasets suggests that machine learning techniques can successfully identify variables with high explanatory power for differentiating among tree species, and opens the possibility of addressing largescale forestry questions using optimized remote sensing workflows. A sample workflow for comparing machine learning methods for tree species classification is shown in Fig. 6.3 (Marrs and Ni-Meister 2019). This thesis tested several machine learning algorithms for tree species classification. Chapter 2, chapter 3 
and chapter 4 showed great capability of the Random Forest algorithm for handling multi-source variables, feature importance evaluation and multi-class classification. Chapter 5 compared three one-class classifiers (i.e. OCSVM, BSVM, and Maxent) for the focal species identification. Despite the advantages of machine learning techniques for tree species mapping from remote sensing, our results also indicate that feature selection and parameters tuning should be carefully investigated for optimizing the classification performance. Future research is needed to further investigate the uncertainties and transferability of machine learning techniques for tree species mapping.

As machine learning is still an active area of research in remote sensing and the wider research community, new developments and new algorithms are likely to provide enhanced and improved functionality for tree species classification from remotely sensed data (Maxwell et al. 2018). For example, deep learning methods (e.g. deep neural networks) allow for new input features to be produced from the low-level input data, such as image bands, that can more adequately describe the classes of interest. 


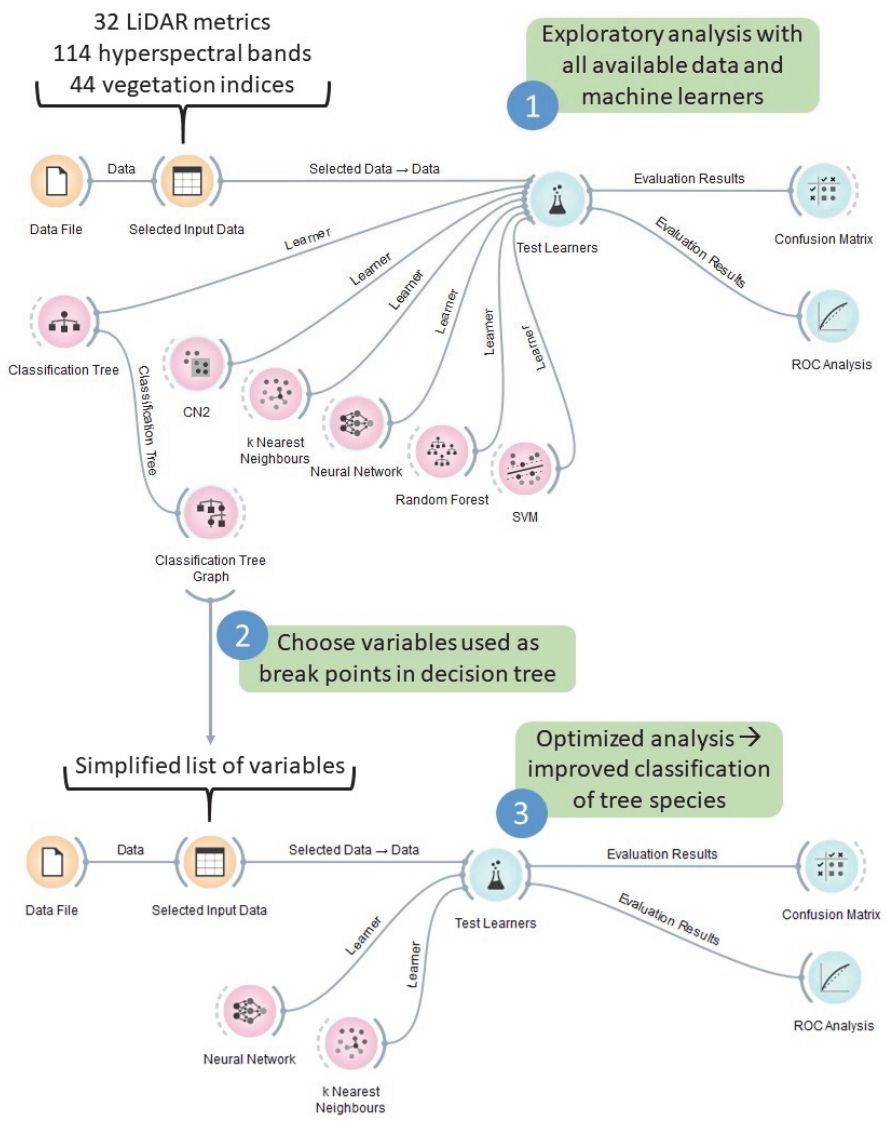

Fig. 6.3 Sample workflow for comparing machine learning methods for tree species classification (Marrs and Ni-Meister 2019).

\subsection{Applications of remote sensing in forestry improve the efficiency of government management and industry}

Remote sensing based mapping of forests can improve the efficiency of forestry management at multiple scales. It is essential that the spatial and temporal resolution of remotely sensed data be matched to the relevant scales of biodiversity, major threats, and management actions (Wiens et al. 2009). At a global scale, remote sensing based mapping of forests can build the foundation for setting spatial priorities about where to focus conservation efforts, assessing the condition of ecological systems, and evaluating the effectiveness of management practices or strategies (Hoekstra et al. 2005). At a regional scale, remote sensing based mapping of forests provides valuable information for 
national forest inventories which can also be updated accordingly for forest monitoring purposes. At a local scale, remote sensing based mapping of forests can display detailed forest characters and parameters at individual tree level, aiding site-specific and species-specific applications.

Remote sensing based mapping of forests assists efficient forestry management in long run. With the advances in spatial, spectral and temporal resolution of remotely sensed data, tree species composition and distribution can be mapped and monitored in various scales with long time span, deriving valuable information for biodiversity assessment and conservation planning. For example, time-series forest maps help managers identify early warning signs of climate change based on early identifications of changes in plant physiology and phenology (Nagendra et al. 2013). Meanwhile, remote sensing can be used to establish spatial priorities, assess the condition of ecological systems, and evaluate the effectiveness of management practices or strategies, as indicated by Wiens et al. (2009). Furthermore, remote sensing based mapping of forests is being used to assess forest cover changes observed across large areas with different forest types and management goals, while still providing high-quality, comparable data that allow characterization, quantification and interpretation of changes based on site-specific conservation objectives.

\subsection{Future work and broader implications}

Individual tree species classification in natural forests remains a challenging task. The ability to accurately discriminate tree species on local to global scales represents a major advance in remote sensing science (Asner 2013). However, success depends, in large part, on a solid understanding of the spectral, spatial and temporal resolution constraints on mapping species within and across a diverse set of ecosystems. Future work should be focusing on discovering and employing species-specific biophysical and biochemical plant traits to improve the interpretability of remotely sensed data for tree species mapping.

Recently, small-format hyperspectral cameras on-board unmanned aerial vehicles (UAVs) have been on the spotlight and they began to be explored for tree species classification in forests (Nevalainen et al. 2017; Tuominen et al. 2018). Compared to satellite and airborne data acquisition, UAV-borne methods have many advantages, such as the possibility to collect data even under poor imaging conditions (e.g. under cloud cover), which makes it very operational in a wide range of environmental measuring applications (Sothe et al. 2019); the cost-efficient data collection with the desired spatial and temporal resolutions; 
and the non-dependence on airports for take-off, or satellite availability in the desired area (Paneque-Gálvez et al. 2014). As they operate at a lower flight altitude than conventional aerial platforms, they offer a finer spatial resolution. On the other hand, UAVs have limited payload, short flight endurance and they present instability in windy conditions, which restrict their use in large scale applications (Nex and Remondino 2014).

Due to increased information on plant phenology, dense time series of multispectral Landsat- 8 and Sentinel-2 data can serve as a good basis for the mapping of forest composition at national scales, considering major tree species typically occurring in temperate and boreal ecosystems (Fassnacht et al. 2016). The WorldView-2 satellite (DigitalGlobe Inc., Westminster, CO, USA), which has eight spectral bands and a spatial resolution of $0.5 \mathrm{~m}$ in the panchromatic band and $2 \mathrm{~m}$ in the VNIR bands, is probably capable to provide relatively accurate estimates of both species diversity and stand structure (Nex and Remondino 2014). Furthermore, the WorldView-3 satellite was launched in August 2014 with a 16-band mode that consists of eight VNIR bands, similar to WorldView-2, in addition to eight short-wave infrared (SWIR) bands that may enhance species-specific analysis (Hartling et al. 2019).

A major aim of several spaceborne hyperspectral missions is to map plant species in support of ecosystem research, including the upcoming NASA's Hyperspectral Infrared Imager, and EnMAP (Environmental Monitoring and Analysis Program) (Abrams and Hook 2013) as well as the ongoing Global Ecosystem Dynamics Investigator (GEDI) spaceborne LiDAR which provides the first detailed 3D structure of forested ecosystems. The relative high resolution and global coverage with revisit observations indicate the potential ability of combining spaceborne hyperspectral and LiDAR sensors for mapping dominant tree species at pixel level in different forest ecosystems. By combining the spaceborne data sets, we attempt to explore their capacity to map tree species across multiple ecosystems simultaneously, which will be the goal for the ongoing and upcoming spaceborne hyperspectral and LiDAR data. 


\section{Bibliography}

Aber, J.S., Marzolff, I., \& Ries, J. (2010). Small-format aerial photography. Elsevier

Abrams, M.J., \& Hook, S.J. (2013). NASA's hyperspectral infrared imager (HyspIRI). Thermal infrared remote sensing (pp. 117-130): Springer

Ali, A.M., Darvishzadeh, R., Skidmore, A.K., \& van Duren, I. (2016a). Effects of canopy structural variables on retrieval of leaf dry matter content and specific leaf area from remotely sensed data. IEEE Journal of Selected Topics in Applied Earth Observations and Remote Sensing, 9, 898-909

Ali, A.M., Darvishzadeh, R., Skidmore, A.K., \& van Duren, I. (2017). Specific leaf area estimation from leaf and canopy reflectance through optimization and validation of vegetation indices. Agricultural and Forest Meteorology, 236, 162-174

Ali, A.M., Skidmore, A.K., Darvishzadeh, R., van Duren, I., Holzwarth, S., \& Mueller, J. (2016b). Retrieval of forest leaf functional traits from HySpex imagery using radiative transfer models and continuous wavelet analysis. ISPRS Journal of Photogrammetry and Remote Sensing, 122, 68-80

Alonzo, M., Bookhagen, B., \& Roberts, D.A. (2014). Urban tree species mapping using hyperspectral and lidar data fusion. Remote Sensing of Environment, $148,70-83$

Andersen, H.-E., McGaughey, R.J., \& Reutebuch, S.E. (2005). Estimating forest canopy fuel parameters using LIDAR data. Remote Sensing of Environment, 94, 441-449

Apan, A., Held, A., Phinn, S., \& Markley, J. (2004). Detecting sugarcane 'orange rust'disease using EO-1 Hyperion hyperspectral imagery. International Journal of Remote Sensing, 25, 489-498

Asner, G.P. (1998). Biophysical and Biochemical Sources of Variability in Canopy Reflectance. Remote Sensing of Environment, 64, 234-253

Asner, G.P. (2007). Carnegie Airborne Observatory: in-flight fusion of hyperspectral imaging and waveform light detection and ranging for threedimensional studies of ecosystems. Journal of Applied Remote Sensing, 1, 013536

Asner, G.P. (2013). Biological diversity mapping comes of age. Remote Sensing, 5, 374-376

Asner, G.P., Knapp, D.E., Kennedy-Bowdoin, T., Jones, M.O., Martin, R.E., Boardman, J., \& Hughes, R.F. (2008). Invasive species detection in Hawaiian 
rainforests using airborne imaging spectroscopy and LiDAR. Remote Sensing of Environment, 112, 1942-1955

Asner, G.P., \& Martin, R.E. (2009). Airborne spectranomics: mapping canopy chemical and taxonomic diversity in tropical forests. Frontiers in Ecology and the Environment, 7, 269-276

Asner, G.P., \& Martin, R.E. (2011). Canopy phylogenetic, chemical and spectral assembly in a lowland Amazonian forest. New Phytologist, 189, 999-1012

Asner, G.P., Martin, R.E., Ford, A.J., Metcalfe, D.J., \& Liddell, M.J. (2009). Leaf chemical and spectral diversity in Australian tropical forests. Ecological Applications, 19, 236-253

Aspinall, R.J. (2002). Use of logistic regression for validation of maps of the spatial distribution of vegetation species derived from high spatial resolution hyperspectral remotely sensed data. Ecological Modelling, 157, 301-312

Atzberger, C. (2000). Development of an invertible forest reflectance model: The INFOR-Model. A decade of trans-European remote sensing cooperation, 3944

Baldeck, C.A., \& Asner, G.P. (2015). Single-Species Detection With Airborne Imaging Spectroscopy Data: A Comparison of Support Vector Techniques. IEEE Journal of Selected Topics in Applied Earth Observations and Remote Sensing, 8, 2501-2512

Baldeck, C.A., Asner, G.P., Martin, R.E., Anderson, C.B., Knapp, D.E., Kellner, J.R., \& Wright, S.J. (2015). Operational tree species mapping in a diverse tropical forest with airborne imaging spectroscopy. PLoS One, 10, e0118403

Barrett, F., McRoberts, R.E., Tomppo, E., Cienciala, E., \& Waser, L.T. (2016). A questionnaire-based review of the operational use of remotely sensed data by national forest inventories. Remote Sensing of Environment, 174, 279-289

Belgiu, M., \& Drăguţ, L. (2016). Random forest in remote sensing: A review of applications and future directions. ISPRS Journal of Photogrammetry and Remote Sensing, 114, 24-31

Blackburn, G.A. (1998). Quantifying Chlorophylls and Caroteniods at Leaf and Canopy Scales: An Evaluation of Some Hyperspectral Approaches. Remote Sensing of Environment, 66, 273-285

Blackburn, G.A. (2007). Hyperspectral remote sensing of plant pigments. $J$ Exp Bot, 58, 855-867

Boschetti, M., Boschetti, L., Oliveri, S., Casati, L., \& Canova, I. (2007). Tree species mapping with Airborne hyper-spectral MIVIS data: the Ticino Park study case. International Journal of Remote Sensing, 28, 1251-1261 
Bouvier, M., Durrieu, S., Fournier, R.A., \& Renaud, J.P. (2015). Generalizing predictive models of forest inventory attributes using an area-based approach with airborne LiDAR data. Remote Sensing of Environment, 156, 322-334

Brandtberg, T. (2007). Classifying individual tree species under leaf-off and leafon conditions using airborne lidar. ISPRS Journal of Photogrammetry and Remote Sensing, 61, 325-340

Brandtberg, T., Warner, T.A., Landenberger, R.E., \& McGraw, J.B. (2003). Detection and analysis of individual leaf-off tree crowns in small footprint, high sampling density lidar data from the eastern deciduous forest in North America. Remote Sensing of Environment, 85, 290-303

Breiman, L. (2001). Random Forests. Machine learning, 45

Broge, N.H., \& Leblanc, E. (2001). Comparing prediction power and stability of broadband and hyperspectral vegetation indices for estimation of green leaf area index and canopy chlorophyll density. Remote Sensing of Environment, 76, 156-172

Budei, B.C., St-Onge, B., Hopkinson, C., \& Audet, F.A. (2018). Identifying the genus or species of individual trees using a three-wavelength airborne lidar system. Remote Sensing of Environment, 204, 632-647

Büntgen, U., Tegel, W., Kaplan, J.O., Schaub, M., Hagedorn, F., Bürgi, M., Brázdil, R., Helle, G., Carrer, M., Heussner, K.-U., Hofmann, J., Kontic, R., Kyncl, T., Kyncl, J., Camarero, J.J., Tinner, W., Esper, J., \& Liebhold, A. (2014). Placing unprecedented recent fir growth in a European-wide and Holocene-long context. Frontiers in Ecology and the Environment, 12, 100106

Cailleret, M., Heurich, M., \& Bugmann, H. (2014). Reduction in browsing intensity may not compensate climate change effects on tree species composition in the Bavarian Forest National Park. Forest ecology and management, 328, 179-192

Calle, Z., Schlumpberger, B.O., Piedrahita, L., Leftin, A., Hammer, S.A., Tye, A., \& Borchert, R. (2010). Seasonal variation in daily insolation induces synchronous bud break and flowering in the tropics. Trees, 24, 865-877

Cao, L., Coops, N.C., Innes, J.L., Dai, J., Ruan, H., \& She, G. (2016). Tree species classification in subtropical forests using small-footprint full-waveform LiDAR data. International Journal of Applied Earth Observation and Geoinformation, 49, 39-51

Carter, G.A. (1994). Ratios of leaf reflectances in narrow wavebands as indicators of plant stress. Remote Sensing, 15, 697-703 
Carter, G.A., \& Knapp, A.K. (2001). Leaf optical properties in higher plants: linking spectral characteristics to stress and chlorophyll concentration. American Journal of Botany, 88, 677-684

Carter, G.A., \& Miller, R.L. (1994). Early detection of plant stress by digital imaging within narrow stress-sensitive wavebands. Remote Sensing of Environment, 50, 295-302

Casas, A., Riano, D., Ustin, S.L., Dennison, P., \& Salas, J. (2014). Estimation of water-related biochemical and biophysical vegetation properties using multitemporal airborne hyperspectral data and its comparison to MODIS spectral response. Remote Sensing of Environment, 148, 28-41

Cavender-Bares, J., Meireles, J.E., Couture, J.J., Kaproth, M.A., Kingdon, C.C., Singh, A., Serbin, S.P., Center, A., Zuniga, E., \& Pilz, G. (2016). Associations of leaf spectra with genetic and phylogenetic variation in oaks: Prospects for remote detection of biodiversity. Remote Sensing, 8, 221

Chen, C., Liaw, A., \& Breiman, L. (2004). Using random forest to learn imbalanced data. University of California, Berkeley, 110

Cho, M.A., Debba, P., Mathieu, R., Naidoo, L., Van Aardt, J., \& Asner, G.P. (2010). Improving discrimination of savanna tree species through a multipleendmember spectral angle mapper approach: Canopy-level analysis. IEEE Transactions on Geoscience and Remote Sensing, 48, 4133-4142

Cho, M.A., Mathieu, R., Asner, G.P., Naidoo, L., van Aardt, J., Ramoelo, A., Debba, P., Wessels, K., Main, R., Smit, I.P.J., \& Erasmus, B. (2012). Mapping tree species composition in South African savannas using an integrated airborne spectral and LiDAR system. Remote Sensing of Environment, 125, 214-226

Cho, M.A., \& Skidmore, A.K. (2006). A new technique for extracting the red edge position from hyperspectral data: The linear extrapolation method. Remote Sensing of Environment, 101, 181-193

Chuine, I., \& Beaubien, E.G. (2001). Phenology is a major determinant of tree species range. Ecology Letters, 4, 500-510

Clark, M., Roberts, D., \& Clark, D. (2005). Hyperspectral discrimination of tropical rain forest tree species at leaf to crown scales. Remote Sensing of Environment, 96, 375-398

Clark, M.L., Clark, D.B., \& Roberts, D.A. (2004). Small-footprint lidar estimation of sub-canopy elevation and tree height in a tropical rain forest landscape. Remote Sensing of Environment, 91, 68-89

Clausi, D.A. (2002). An analysis of co-occurrence texture statistics as a function of grey level quantization. Canadian Journal of Remote Sensing, 28, 45-62 
Clevers, J.G.P.W., Kooistra, L., \& Schaepman, M.E. (2010). Estimating canopy water content using hyperspectral remote sensing data. International Journal of Applied Earth Observation and Geoinformation, 12, 119-125

Cohen, J. (1960). A coefficient of agreement for nominal scales. Educational and psychological measurement, 20, 37-46

Colwell, R.N. (1960). Manual of photographic interpretation. American Society of Photogrammetry

Coops, N.C., Hilker, T., Wulder, M.A., St-Onge, B., Newnham, G., Siggins, A., \& Trofymow, J.T. (2007). Estimating canopy structure of Douglas-fir forest stands from discrete-return LiDAR. Trees, 21, 295-310

Coops, N.C., Wulder, M.A., Culvenor, D.S., \& St-Onge, B. (2004). Comparison of forest attributes extracted from fine spatial resolution multispectral and lidar data. Canadian Journal of Remote Sensing, 30, 855-866

Cootes, T.F., Ionita, M.C., Lindner, C., \& Sauer, P. (2012). Robust and Accurate Shape Model Fitting Using Random Forest Regression Voting. In A. Fitzgibbon, S. Lazebnik, P. Perona, Y. Sato, \& C. Schmid (Eds.), Computer Vision - ECCV 2012: 12th European Conference on Computer Vision, Florence, Italy, October 7-13, 2012, Proceedings, Part VII (pp. 278-291). Berlin, Heidelberg: Springer Berlin Heidelberg

Cornelissen, J.H.C., Lavorel, S., Garnier, E., Díaz, S., Buchmann, N., Gurvich, D.E., Reich, P.B., Steege, H.t., Morgan, H.D., Heijden, M.G.A.v.d., Pausas, J.G., \& Poorter, H. (2003). A handbook of protocols for standardised and easy measurement of plant functional traits worldwide. Australian Journal of Botany, 51, 335-380

Dalponte, M., Bruzzone, L., \& Gianelle, D. (2008). Fusion of hyperspectral and LIDAR remote sensing data for classification of complex forest areas. Geoscience and Remote Sensing, IEEE Transactions on, 46, 1416-1427

Dalponte, M., Bruzzone, L., \& Gianelle, D. (2012). Tree species classification in the Southern Alps based on the fusion of very high geometrical resolution multispectral/hyperspectral images and LiDAR data. Remote Sensing of Environment, 123, 258-270

Dalponte, M., Frizzera, L., \& Gianelle, D. (2019). Individual tree crown delineation and tree species classification with hyperspectral and LiDAR data. Peerj, 6

Dalponte, M., Orka, H.O., Ene, L.T., Gobakken, T., \& Naesset, E. (2014). Tree crown delineation and tree species classification in boreal forests using hyperspectral and ALS data. Remote Sensing of Environment, 140, 306-317 
Dalponte, M., Orka, H.O., Gobakken, T., Gianelle, D., \& Næsset, E. (2013). Tree species classification in boreal forests with hyperspectral data. Geoscience and Remote Sensing, IEEE Transactions on, 51, 2632-2645

Darvishzadeh, R., Skidmore, A., Schlerf, M., \& Atzberger, C. (2008). Inversion of a radiative transfer model for estimating vegetation LAI and chlorophyll in a heterogeneous grassland. Remote Sensing of Environment, 112, 2592-2604

Datt, B. (1999). A New Reflectance Index for Remote Sensing of Chlorophyll Content in Higher Plants: Tests using Eucalyptus Leaves. Journal of Plant Physiology, 154, 30-36

de Leeuw, J., Jia, H., Yang, L., Liu, X., Schmidt, K., \& Skidmore, A.K. (2006). Comparing accuracy assessments to infer superiority of image classification methods. International Journal of Remote Sensing, 27, 223-232

Debba, P., Cho, M.A., \& Mathieu, R. (2009). Within- and between-class variability of spectrally similar tree species. In, 2009 IEEE International Geoscience and Remote Sensing Symposium (pp. IV-272-IV-275)

Dechesne, C., Mallet, C., Le Bris, A., \& Gouet-Brunet, V. (2017). Semantic segmentation of forest stands of pure species combining airborne lidar data and very high resolution multispectral imagery. ISPRS Journal of Photogrammetry and Remote Sensing, 126, 129-145

Deng, S., Katoh, M., Yu, X., Hyyppä, J., \& Gao, T. (2016). Comparison of Tree Species Classifications at the Individual Tree Level by Combining ALS Data and RGB Images Using Different Algorithms. Remote Sensing, 8, 1034

Desplanque, C., Rolland, C., \& Schweingruber, F.H. (1999). Influence of species and abiotic factors on extreme tree ring modulation. Trees, 13, 218-227

Dobrowolska, D., Bončina, A., \& Klumpp, R. (2017). Ecology and silviculture of silver fir (Abies alba Mill.): A review. Journal of Forest Research, 22, 326335

Dormann, C.F., Elith, J., Bacher, S., Buchmann, C., Carl, G., Carré, G., Marquéz, J.R.G., Gruber, B., Lafourcade, B., Leitão, P.J., Münkemüller, T., McClean, C., Osborne, P.E., Reineking, B., Schröder, B., Skidmore, A.K., Zurell, D., \& Lautenbach, S. (2013). Collinearity: a review of methods to deal with it and a simulation study evaluating their performance. Ecography, 36, 27-46

Dudley, K.L., Dennison, P.E., Roth, K.L., Roberts, D.A., \& Coates, A.R. (2015). A multi-temporal spectral library approach for mapping vegetation species across spatial and temporal phenological gradients. Remote Sensing of Environment, 167, 121-134

Elling, W., Dittmar, C., Pfaffelmoser, K., \& Rötzer, T. (2009). Dendroecological assessment of the complex causes of decline and recovery of the growth of 
silver fir (Abies alba Mill.) in Southern Germany. Forest ecology and management, 257, 1175-1187

Essery, R., Bunting, P., Rowlands, A., Rutter, N., Hardy, J., Melloh, R., Link, T., Marks, D., \& Pomeroy, J. (2008). Radiative transfer modeling of a coniferous canopy characterized by airborne remote sensing. Journal of Hydrometeorology, 9, 228-241

Fang, F., McNeil, B.E., Warner, T.A., \& Maxwell, A.E. (2018). Combining high spatial resolution multi-temporal satellite data with leaf-on LiDAR to enhance tree species discrimination at the crown level. International Journal of Remote Sensing, 39, 9054-9072

Farjon, A. (1990). Pinaceae. Drawings and descriptions of the genera Abies, Cedrus, Pseudolarix, Keteleeria, Nothotsuga, Tsuga, Cathaya, Pseudotsuga, Larix and Picea. Koeltz scientific books

Fassnacht, F.E., Latifi, H., Stereńczak, K., Modzelewska, A., Lefsky, M., Waser, L.T., Straub, C., \& Ghosh, A. (2016). Review of studies on tree species classification from remotely sensed data. Remote Sensing of Environment, 186, 64-87

Fassnacht, F.E., Stenzel, S., \& Gitelson, A.A. (2015). Non-destructive estimation of foliar carotenoid content of tree species using merged vegetation indices. Journal of Plant Physiology, 176, 210-217

Feilhauer, H., Asner, G.P., \& Martin, R.E. (2015). Multi-method ensemble selection of spectral bands related to leaf biochemistry. Remote Sensing of Environment, 164, 57-65

Feret, J.-B., \& Asner, G.P. (2013). Tree species discrimination in tropical forests using airborne imaging spectroscopy. Geoscience and Remote Sensing, IEEE Transactions on, 51, 73-84

Féret, J.-B., \& Asner, G.P. (2011). Spectroscopic classification of tropical forest species using radiative transfer modeling. Remote Sensing of Environment, $115,2415-2422$

Ferreira, M.P., Féret, J.-B., Grau, E., Gastellu-Etchegorry, J.-P., do Amaral, C.H., Shimabukuro, Y.E., \& de Souza Filho, C.R. (2018). Retrieving structural and chemical properties of individual tree crowns in a highly diverse tropical forest with 3D radiative transfer modeling and imaging spectroscopy. Remote Sensing of Environment, 211, 276-291

Ferreira, M.P., Zortea, M., Zanotta, D.C., Shimabukuro, Y.E., \& de Souza Filho, C.R. (2016). Mapping tree species in tropical seasonal semi-deciduous forests with hyperspectral and multispectral data. Remote Sensing of Environment, $179,66-78$ 
Ficko, A., Poljanec, A., \& Boncina, A. (2011). Do changes in spatial distribution, structure and abundance of silver fir (Abies alba Mill.) indicate its decline? Forest ecology and management, 261, 844-854

Galvão, L.S., Formaggio, A.R., \& Tisot, D.A. (2005). Discrimination of sugarcane varieties in Southeastern Brazil with EO-1 Hyperion data. Remote Sensing of Environment, 94, 523-534

Gamon, J., \& Surfus, J. (1999). Assessing leaf pigment content and activity with a reflectometer. The New Phytologist, 143, 105-117

Gamon, J.A., Field, C.B., Goulden, M.L., Griffin, K.L., Hartley, A.E., Joel, G., Peñuelas, J., \& Valentini, R. (1995). Relationships between NDVI, canopy structure, and photosynthesis in three Californian vegetation types. Ecological Applications, 5, 28-41

Ganivet, E., \& Bloomberg, M. (2019). Towards rapid assessments of tree species diversity and structure in fragmented tropical forests: A review of perspectives offered by remotely-sensed and field-based data. Forest ecology and management, 432, 40-53

Gärtner, P., Förster, M., \& Kleinschmit, B. (2016). The benefit of synthetically generated RapidEye and Landsat 8 data fusion time series for riparian forest disturbance monitoring. Remote Sensing of Environment, 177, 237-247

Gaveau, D.L., \& Hill, R.A. (2003). Quantifying canopy height underestimation by laser pulse penetration in small-footprint airborne laser scanning data. Canadian Journal of Remote Sensing, 29, 650-657

Gege, P., Fries, J., Haschberger, P., Schötz, P., Schwarzer, H., Strobl, P., Suhr, B., Ulbrich, G., \& Jan Vreeling, W. (2009). Calibration facility for airborne imaging spectrometers. ISPRS Journal of Photogrammetry and Remote Sensing, 64, 387-397

Ghiyamat, A., \& Shafri, H.Z.M. (2010). A review on hyperspectral remote sensing for homogeneous and heterogeneous forest biodiversity assessment. International Journal of Remote Sensing, 31, 1837-1856

Ghosh, A., Fassnacht, F.E., Joshi, P.K., \& Koch, B. (2014). A framework for mapping tree species combining hyperspectral and LiDAR data: Role of selected classifiers and sensor across three spatial scales. International Journal of Applied Earth Observation and Geoinformation, 26, 49-63

Gitelson, A.A., Gritz †, Y., \& Merzlyak, M.N. (2003a). Relationships between leaf chlorophyll content and spectral reflectance and algorithms for nondestructive chlorophyll assessment in higher plant leaves. Journal of Plant Physiology, 160, 271-282 
Gitelson, A.A., Vina, A., Arkebauer, T.J., Rundquist, D.C., Keydan, G., \& Leavitt, B. (2003b). Remote estimation of leaf area index and green leaf biomass in maize canopies. Geophysical Research Letters, 30

Große-Stoltenberg, A., Hellmann, C., Werner, C., Oldeland, J., \& Thiele, J. (2016). Evaluation of continuous VNIR-SWIR spectra versus narrowband hyperspectral indices to discriminate the invasive Acacia longifolia within a Mediterranean dune ecosystem. Remote Sensing, 8, 334

Haara, A., \& Haarala, M. (2002). Tree species classification using semiautomatic delineation of trees on aerial images. Scandinavian Journal of Forest Research, 17, 556-565

Haralick, R.M., Shanmugam, K., \& Dinstein, I. (1973). Textural Features for Image Classification. IEEE Transactions on Systems, Man, and Cybernetics, SMC-3, 610-621

Hartling, S., Sagan, V., Sidike, P., Maimaitijiang, M., \& Carron, J. (2019). Urban Tree Species Classification Using a WorldView-2/3 and LiDAR Data Fusion Approach and Deep Learning. Sensors (Basel, Switzerland), 19, 1284

Heikkinen, V., Tokola, T., Parkkinen, J., Korpela, I., \& Jääskeläinen, T. (2010). Simulated multispectral imagery for tree species classification using support vector machines. IEEE Transactions on Geoscience and Remote Sensing, 48, 1355

Heinzel, J., \& Koch, B. (2011). Exploring full-waveform LiDAR parameters for tree species classification. International Journal of Applied Earth Observation and Geoinformation, 13, 152-160

Heinzel, J., \& Koch, B. (2012). Investigating multiple data sources for tree species classification in temperate forest and use for single tree delineation. International Journal of Applied Earth Observation and Geoinformation, 18, 101-110

Heinzel, J.N., Weinacker, H., \& Koch, B. (2008). Full automatic detection of tree species based on delineated single tree crowns-a data fusion approach for airborne laser scanning data and aerial photographs. Proceedings of SILVILASER, 2008, 8th

Hernández-Clemente, R., Navarro-Cerrillo, R.M., Suárez, L., Morales, F., \& Zarco-Tejada, P.J. (2011). Assessing structural effects on PRI for stress detection in conifer forests. Remote Sensing of Environment, 115, 2360-2375

Heurich, M. (2006). Evaluierung und entwicklung von automatiserten erfassung von waldstructuren aus daten flugzuggetragener fernerkundungssenoren. Forstkuche Forshungsberichte München, 2002, p329 
Heurich, M. (2008). Automatic recognition and measurement of single trees based on data from airborne laser scanning over the richly structured natural forests of the Bavarian Forest National Park. Forest ecology and management, $255,2416-2433$

Heurich, M., Beudert, B., Rall, H., \& Křenová, Z. (2010). National parks as model regions for interdisciplinary long-term ecological research: the Bavarian Forest and Šumavá National Parks underway to transboundary ecosystem research. Long-Term Ecological Research (pp. 327-344): Springer

Heurich, M., \& Englmaier, K.H. (2010). The development of tree species composition in the Rachel-Lusen region of the Bavarian Forest National Park. Silva Gabreta, 16, 165-186

Heurich, M., \& Neufanger, M. (2005). Die Wälder des Nationalparks Bayerischer Wald: Ergebnisse der Waldinventur 2002/2003 im Geschichtlichen und Waldökologischen Kontext. Nationalparkverwaltung Bayerischer Wald

Heuze, P., Schnitzler, A., \& Klein, F. (2005). Consequences of increased deer browsing winter on silver fir and spruce regeneration in the Southern Vosges mountains: Implications for forest management. Annals of Forest Science, 62, 175-181

Hoekstra, J.M., Boucher, T.M., Ricketts, T.H., \& Roberts, C. (2005). Confronting a biome crisis: global disparities of habitat loss and protection. Ecology Letters, 8, 23-29

Höfle, B., Hollaus, M., \& Hagenauer, J. (2012). Urban vegetation detection using radiometrically calibrated small-footprint full-waveform airborne LiDAR data. ISPRS Journal of Photogrammetry and Remote Sensing, 67, 134-147

Holmgren, J., \& Persson, Å. (2004). Identifying species of individual trees using airborne laser scanner. Remote Sensing of Environment, 90, 415-423

Holmgren, J., Persson, Å., \& Söderman, U. (2008). Species identification of individual trees by combining high resolution LiDAR data with multi-spectral images. International Journal of Remote Sensing, 29, 1537-1552

Holmgren, P., \& Thuresson, T. (1998). Satellite remote sensing for forestry planning - a review. Scandinavian Journal of Forest Research, 13, 90-110

Homolová, L., Malenovský, Z., Clevers, J.G.P.W., García-Santos, G., \& Schaepman, M.E. (2013). Review of optical-based remote sensing for plant trait mapping. Ecological Complexity, 15, 1-16

Hopkinson, C., Chasmer, L., Lim, K., Treitz, P., \& Creed, I. (2006). Towards a universal lidar canopy height indicator. Canadian Journal of Remote Sensing, $32,139-152$ 
Hovi, A., Korhonen, L., Vauhkonen, J., \& Korpela, I. (2016). LiDAR waveform features for tree species classification and their sensitivity to tree- and acquisition related parameters. Remote Sensing of Environment, 173, 224-237

Huber, S., Kneubuhler, M., Psomas, A., Itten, K., \& Zimmermann, N.E. (2008). Estimating foliar biochemistry from hyperspectral data in mixed forest canopy. Forest ecology and management, 256, 491-501

Hyyppä, J., Kelle, O., Lehikoinen, M., \& Inkinen, M. (2001). A segmentationbased method to retrieve stem volume estimates from 3-D tree height models produced by laser scanners. Geoscience and Remote Sensing, IEEE Transactions on, 39, 969-975

Immitzer, M., Atzberger, C., \& Koukal, T. (2012). Tree Species Classification with Random Forest Using Very High Spatial Resolution 8-Band WorldView2 Satellite Data. Remote Sensing, 4, 2661-2693

Jacquemoud, S., Ustin, S., Verdebout, J., Schmuck, G., Andreoli, G., \& Hosgood, B. (1996). Estimating leaf biochemistry using the PROSPECT leaf optical properties model. Remote Sensing of Environment, 56, 194-202

Jakubowski, M.K., Li, W., Guo, Q., \& Kelly, M. (2013). Delineating individual trees from LiDAR data: A comparison of vector-and raster-based segmentation approaches. Remote Sensing, 5, 4163-4186

Jones, T.G., Coops, N.C., \& Sharma, T. (2010). Assessing the utility of airborne hyperspectral and LiDAR data for species distribution mapping in the coastal Pacific Northwest, Canada. Remote Sensing of Environment, 114, 2841-2852

Kamal, M., \& Phinn, S. (2011). Hyperspectral Data for Mangrove Species Mapping: A Comparison of Pixel-Based and Object-Based Approach. Remote Sensing, 3, 2222-2242

Kayitakire, F., Hamel, C., \& Defourny, P. (2006). Retrieving forest structure variables based on image texture analysis and IKONOS-2 imagery. Remote Sensing of Environment, 102, 390-401

Key, T., Warner, T.A., McGraw, J.B., \& Fajvan, M.A. (2001). A comparison of multispectral and multitemporal information in high spatial resolution imagery for classification of individual tree species in a temperate hardwood forest. Remote Sensing of Environment, 75, 100-112

Kim, S. (2007). Individual tree species identification using LIDAR-derived crown structures and intensity data. In: University of Washington

Kim, S., Hinckley, T., \& Briggs, D. (2011). Classifying individual tree genera using stepwise cluster analysis based on height and intensity metrics derived from airborne laser scanner data. Remote Sensing of Environment, 115, 33293342 
Kim, S., McGaughey, R.J., Andersen, H.E., \& Schreuder, G. (2009). Tree species differentiation using intensity data derived from leaf-on and leaf-off airborne laser scanner data. Remote Sensing of Environment, 113, 1575-1586

Kirby, K.R., \& Potvin, C. (2007). Variation in carbon storage among tree species: Implications for the management of a small-scale carbon sink project. Forest ecology and management, 246, 208-221

Klopčič, M., Mina, M., Bugmann, H., \& Bončina, A. (2017). The prospects of silver fir (Abies alba Mill.) and Norway spruce (Picea abies (L.) Karst) in mixed mountain forests under various management strategies, climate change and high browsing pressure. European Journal of Forest Research, 136, 10711090

Knowlton, D., \& Hoffer, R. (1981). Radar imagery for forest cover mapping

Koch, B., Svoboda, J., Adler, P., \& Dees, M. (2002). Automatic tree species detection based on digitised CIR aerial photos. Allgemeine Forst Und Jagdzeitung, 173, 131-140

Koenig, K., \& Höfle, B. (2016). Full-Waveform Airborne Laser Scanning in Vegetation Studies-A Review of Point Cloud and Waveform Features for Tree Species Classification. Forests, 7, 198

Köhler, C.H. (2016). Airborne Imaging Spectrometer HySpex. Journal of largescale research facilities $J L S R F, 2,1-6$

Kokaly, R.F., Asner, G.P., Ollinger, S.V., Martin, M.E., \& Wessman, C.A. (2009). Characterizing canopy biochemistry from imaging spectroscopy and its application to ecosystem studies. Remote Sensing of Environment, 113, Supplement 1, S78-S91

Kokaly, R.F., \& Clark, R.N. (1999). Spectroscopic determination of leaf biochemistry using band-depth analysis of absorption features and stepwise multiple linear regression. Remote Sensing of Environment, 67, 267-287

Korpela, I. (2006). Incorporation of allometry into single-tree remote sensing with lidar and multiple aerial images. Department of Forest Resource Management. University of Helsinki, Finland, 6

Korpela, I., Heikkinen, V., Honkavaara, E., Rohrbach, F., \& Tokola, T. (2011). Variation and directional anisotropy of reflectance at the crown scaleImplications for tree species classification in digital aerial images. Remote Sensing of Environment, 115, 2062-2074

Korpela, I., Hovi, A., \& Korhonen, L. (2013). Backscattering of individual LiDAR pulses from forest canopies explained by photogrammetrically derived vegetation structure. ISPRS Journal of Photogrammetry and Remote Sensing, 83, 81-93 
Korpela, I., Mehtätalo, L., Markelin, L., Seppänen, A., \& Kangas, A. (2014). Tree species identification in aerial image data using directional reflectance signatures. Silva Fenn, 48, 1-20

Korpela, I., Ørka, H.O., Maltamo, M., Tokola, T., \& Hyyppä, J. (2010). Tree species classification using airborne LiDAR-effects of stand and tree parameters, downsizing of training set, intensity normalization, and sensor type. Silva Fennica, 44, 319-339

Kötz, B., Schaepman, M., Morsdorf, F., Bowyer, P., Itten, K., \& Allgöwer, B. (2004). Radiative transfer modeling within a heterogeneous canopy for estimation of forest fire fuel properties. Remote Sensing of Environment, 92, 332-344

Koukoulas, S., \& Blackburn, G.A. (2005). Mapping individual tree location, height and species in broadleaved deciduous forest using airborne LIDAR and multi-spectral remotely sensed data. International Journal of Remote Sensing, $26,431-455$

Kozhoridze, G., Orlovsky, N., Orlovsky, L., Blumberg, D.G., \& Golan-Goldhirsh, A. (2016). Remote sensing models of structure-related biochemicals and pigments for classification of trees. Remote Sensing of Environment, 186, 184195

Kulikova, M.S., Mani, M., Srivastava, A., Descombes, X., \& Zerubia, J. (2007). Tree species classification using radiometry, texture and shape based features. In, Signal Processing Conference, 2007 15th European (pp. 1595-1599): IEEE

Kuzmin, A., Korhonen, L., Manninen, T., \& Maltamo, M. (2016). Automatic Segment-Level Tree Species Recognition Using High Resolution Aerial Winter Imagery. European Journal of Remote Sensing, 49, 239-259

Latifi, H., Fassnacht, F., \& Koch, B. (2012). Forest structure modeling with combined airborne hyperspectral and LiDAR data. Remote Sensing of Environment, 121, 10-25

Lebourgeois, F., Gomez, N., Pinto, P., \& Mérian, P. (2013). Mixed stands reduce Abies alba tree-ring sensitivity to summer drought in the Vosges mountains, western Europe. Forest ecology and management, 303, 61-71

Leckie, D.G., Gougeon, F.A., Walsworth, N., \& Paradine, D. (2003). Stand delineation and composition estimation using semi-automated individual tree crown analysis. Remote Sensing of Environment, 85, 355-369

Leckie, D.G., Tinis, S., Nelson, T., Burnett, C., Gougeon, F.A., Cloney, E., \& Paradine, D. (2005). Issues in species classification of trees in old growth conifer stands. Canadian Journal of Remote Sensing, 31, 175-190 
Lefcheck, J.S., Bastazini, V.A., \& Griffin, J.N. (2015). Choosing and using multiple traits in functional diversity research. Environmental Conservation, 42, 104-107

Li, J., Hu, B., \& Noland, T.L. (2013). Classification of tree species based on structural features derived from high density LiDAR data. Agricultural and Forest Meteorology, 171-172, 104-114

Liaw, A., \& Wiener, M. (2002). Classification and Regression by randomForest. $R$ news, $2 / 3$

Lim, J., Kim, K.M., \& Jin, R. (2019). Tree Species Classification Using Hyperion and Sentinel-2 Data with Machine Learning in South Korea and China. Isprs International Journal of Geo-Information, 8

Lin, Y.-C. (2015). Normalization of echo features derived from full-waveform airborne laser scanning data. Remote Sensing, 7, 2731-2751

Lin, Y., \& Herold, M. (2016). Tree species classification based on explicit tree structure feature parameters derived from static terrestrial laser scanning data. Agricultural and Forest Meteorology, 216, 105-114

Lin, Y., \& Hyyppä, J. (2016). A comprehensive but efficient framework of proposing and validating feature parameters from airborne LiDAR data for tree species classification. International Journal of Applied Earth Observation and Geoinformation, 46, 45-55

Lindberg, E., Eysn, L., Hollaus, M., Holmgren, J., \& Pfeifer, N. (2014). Delineation of tree crowns and tree species classification from full-waveform airborne laser scanning data using 3-D ellipsoidal clustering. Selected Topics in Applied Earth Observations and Remote Sensing, IEEE Journal of, 7, 31743181

Liu, B., Dai, Y., Li, X., Lee, W.S., \& Yu, P.S. (2003). Building text classifiers using positive and unlabeled examples. In, Third IEEE International Conference on Data Mining (pp. 179-186)

Liu, L., Coops, N.C., Aven, N.W., \& Pang, Y. (2017). Mapping urban tree species using integrated airborne hyperspectral and LiDAR remote sensing data. Remote Sensing of Environment, 200, 170-182

Liu, Z. (2016). Effects of climate and fire on short-term vegetation recovery in the boreal larch forests of Northeastern China. Scientific reports, 6, 37572

Lobell, D.B., Asner, G.P., Law, B.E., \& Treuhaft, R.N. (2001). Subpixel canopy cover estimation of coniferous forests in Oregon using SWIR imaging spectrometry. Journal of Geophysical Research: Atmospheres, 106, 51515160 
Loetsch, F., \& Haller, K. (1964). Forest inventory. Volume I. Statistics of forest inventory and information from aerial photographs. Forest inventory. Volume I. Statistics of forest inventory and information from aerial photographs.

Mack, B., Roscher, R., \& Waske, B. (2014). Can i trust my one-class classification? Remote Sensing, 6, 8779-8802

Mack, B., \& Waske, B. (2017). In-depth comparisons of MaxEnt, biased SVM and one-class SVM for one-class classification of remote sensing data. Remote Sensing Letters, 8, 290-299

Maghsoudi, Y., Collins, M., \& Leckie, D.G. (2012). Polarimetric classification of Boreal forest using nonparametric feature selection and multiple classifiers. International Journal of Applied Earth Observation and Geoinformation, 19, 139-150

Mairs, J.W. (1976). The use of remote sensing techniques to identify potential natural areas in oregon. Biological Conservation, 9, 259-266

Maldonado, S., \& Weber, R. (2009). A wrapper method for feature selection using Support Vector Machines. Information Sciences, 179, 2208-2217

Marrs, J., \& Ni-Meister, W. (2019). Machine Learning Techniques for Tree Species Classification Using Co-Registered LiDAR and Hyperspectral Data. Remote Sensing, 11, 819

Martin, R.E., Chadwick, K.D., Brodrick, P.G., Carranza-Jimenez, L., Vaughn, N.R., \& Asner, G.P. (2018). An Approach for Foliar Trait Retrieval from Airborne Imaging Spectroscopy of Tropical Forests. Remote Sensing, 10, 199

Maxwell, A.E., Warner, T.A., \& Fang, F. (2018). Implementation of machinelearning classification in remote sensing: an applied review. International Journal of Remote Sensing, 39, 2784-2817

McNemar, Q. (1947). Note on the sampling error of the difference between correlated proportions or percentages. Psychometrika, 12, 153-157

Merton, R. (1998). Monitoring community hysteresis using spectral shift analysis and the red-edge vegetation stress index. In, Proceedings of the Seventh Annual JPL Airborne Earth Science Workshop (pp. 12-16)

Merton, R.N. (1999). Multi-temporal analysis of community scale vegetation stress with imaging spectroscopy. In: ResearchSpace@ Auckland

Milton, E.J., Schaepman, M.E., Anderson, K., Kneubühler, M., \& Fox, N. (2009). Progress in field spectroscopy. Remote Sensing of Environment, 113, S92S109

Moffiet, T., Mengersen, K., Witte, C., King, R., \& Denham, R. (2005). Airborne laser scanning: Exploratory data analysis indicates potential variables for 
classification of individual trees or forest stands according to species. ISPRS Journal of Photogrammetry and Remote Sensing, 59, 289-309

Morgan, J.L., Gergel, S.E., Ankerson, C., Tomscha, S.A., \& Sutherland, I.J. (2017). Historical Aerial Photography for Landscape Analysis. Learning Landscape Ecology (pp. 21-40): Springer

Müller, R., Lehner, M., Reinartz, P., \& Schroeder, M. (2005). Evaluation of spaceborne and airborne line scanner images using a generic ortho image processor. In, Proc. of High Resolution Earth Imaging for Geospatial Information, ISPRS Hannover Workshop, Commision I WG (p. 2005)

Muñoz-Marí, J., Bovolo, F., Gómez-Chova, L., Bruzzone, L., \& Camp-Valls, G. (2010). Semisupervised one-class support vector machines for classification of remote sensing data. IEEE Transactions on Geoscience and Remote Sensing, 48, 3188-3197

Muss, J.D., Mladenoff, D.J., \& Townsend, P.A. (2011). A pseudo-waveform technique to assess forest structure using discrete lidar data. Remote Sensing of Environment, 115, 824-835

Næsset, E. (2002). Predicting forest stand characteristics with airborne scanning laser using a practical two-stage procedure and field data. Remote Sensing of Environment, 80, 88-99

Naesset, E. (2005). Assessing sensor effects and effects of leaf-off and leaf-on canopy conditions on biophysical stand properties derived from smallfootprint airborne laser data. Remote Sensing of Environment, 98, 356-370

Nagendra, H., Lucas, R., Honrado, J.P., Jongman, R.H.G., Tarantino, C., Adamo, M., \& Mairota, P. (2013). Remote sensing for conservation monitoring: Assessing protected areas, habitat extent, habitat condition, species diversity, and threats. Ecological Indicators, 33, 45-59

Naidoo, L., Cho, M.A., Mathieu, R., \& Asner, G. (2012). Classification of savanna tree species, in the Greater Kruger National Park region, by integrating hyperspectral and LiDAR data in a Random Forest data mining environment. ISPRS Journal of Photogrammetry and Remote Sensing, 69, 167-179

Nevalainen, O., Honkavaara, E., Tuominen, S., Viljanen, N., Hakala, T., Yu, X., Hyyppä, J., Saari, H., Pölönen, I., \& Imai, N. (2017). Individual tree detection and classification with UAV-based photogrammetric point clouds and hyperspectral imaging. Remote Sensing, 9, 185

Nex, F., \& Remondino, F. (2014). UAV for 3D mapping applications: a review. Applied Geomatics, 6, 1-15 
Olden, Julian D., Lawler, Joshua J., \& Poff, N.L. (2008). Machine Learning Methods Without Tears: A Primer for Ecologists. The Quarterly review of biology, 83, 171-193

Onojeghuo, A.O., \& Blackburn, G.A. (2011). Optimising the use of hyperspectral and LiDAR data for mapping reedbed habitats. Remote Sensing of Environment, 115, 2025-2034

Ørka, H.O., Dalponte, M., Gobakken, T., Næsset, E., \& Ene, L.T. (2013). Characterizing forest species composition using multiple remote sensing data sources and inventory approaches. Scandinavian Journal of Forest Research, $28,677-688$

Ørka, H.O., Gobakken, T., Næsset, E., Ene, L., \& Lien, V. (2012). Simultaneously acquired airborne laser scanning and multispectral imagery for individual tree species identification. Canadian Journal of Remote Sensing, $38,125-138$

Ørka, H.O., Næsset, E., \& Bollandsås, O.M. (2009). Classifying species of individual trees by intensity and structure features derived from airborne laser scanner data. Remote Sensing of Environment, 113, 1163-1174

Ørka, H.O., Næsset, E., \& Bollandsås, O.M. (2010). Effects of different sensors and leaf-on and leaf-off canopy conditions on echo distributions and individual tree properties derived from airborne laser scanning. Remote Sensing of Environment, 114, 1445-1461

Ozdemir, I., Norton, D.A., Ozkan, U.Y., Mert, A., \& Senturk, O. (2008). Estimation of tree size diversity using object oriented texture analysis and aster imagery. Sensors, 8, 4709-4724

Palmer, M.W., Earls, P.G., Hoagland, B.W., White, P.S., \& Wohlgemuth, T. (2002). Quantitative tools for perfecting species lists. Environmetrics, 13, 121-137

Paneque-Gálvez, J., McCall, M.K., Napoletano, B.M., Wich, S.A., \& Koh, L.P. (2014). Small Drones for Community-Based Forest Monitoring: An Assessment of Their Feasibility and Potential in Tropical Areas. Forests, 5, 1481-1507

Pasher, J., \& King, D.J. (2010). Multivariate forest structure modelling and mapping using high resolution airborne imagery and topographic information. Remote Sensing of Environment, 114, 1718-1732

Pcorona, A.B., Corona, P., \& Marchetti, M. (2006). European Forest Types Categories and types for sustainable forest management reporting and policy

Pearson, R.G. (2007). Species' distribution modeling for conservation educators and practitioners. Synthesis. American Museum of Natural History, 50, 54-89 
Persson, Å., Holmgren, J., Söderman, U., \& Olsson, H. (2004). Tree species classification of individual trees in Sweden by combining high resolution laser data with high resolution near-infrared digital images. International Archives of Photogrammetry, Remote Sensing and Spatial Information Sciences, 36, 204-207

Phillips, S.J., Anderson, R.P., \& Schapire, R.E. (2006). Maximum entropy modeling of species geographic distributions. Ecological Modelling, 190, 231-259

Phillips, S.J., \& Dudík, M. (2008). Modeling of species distributions with Maxent: new extensions and a comprehensive evaluation. Ecography, 31, 161-175

Piiroinen, R., Fassnacht, F.E., Heiskanen, J., Maeda, E., Mack, B., \& Pellikka, P. (2018). Invasive tree species detection in the Eastern Arc Mountains biodiversity hotspot using one class classification. Remote Sensing of Environment, 218, 119-131

Puttonen, E., Suomalainen, J., Hakala, T., Räikkönen, E., Kaartinen, H., Kaasalainen, S., \& Litkey, P. (2010). Tree species classification from fused active hyperspectral reflectance and LIDAR measurements. Forest ecology and management, 260, 1843-1852

Rautiainen, M., Lukeš, P., Homolová, L., Hovi, A., Pisek, J., \& Mõttus, M. (2018). Spectral Properties of Coniferous Forests: A Review of In Situ and Laboratory Measurements. Remote Sensing, 10, 207

Reitberger, J., Krzystek, P., \& Stilla, U. (2008). Analysis of full waveform LIDAR data for the classification of deciduous and coniferous trees. International Journal of Remote Sensing, 29, 1407-1431

Reitberger, J., Schnorr, C., Krzystek, P., \& Stilla, U. (2009). 3D segmentation of single trees exploiting full waveform LIDAR data. ISPRS Journal of Photogrammetry and Remote Sensing, 64, 561-574

Riaño, D., Chuvieco, E., Condés, S., González-Matesanz, J., \& Ustin, S.L. (2004). Generation of crown bulk density for Pinus sylvestris L. from lidar. Remote Sensing of Environment, 92, 345-352

Ribeiro da Luz, B., \& Crowley, J.K. (2007). Spectral reflectance and emissivity features of broad leaf plants: Prospects for remote sensing in the thermal infrared (8.0-14.0 $\mu \mathrm{m})$. Remote Sensing of Environment, 109, 393-405

Richter, R., Reu, B., Wirth, C., Doktor, D., \& Vohland, M. (2016). The use of airborne hyperspectral data for tree species classification in a species-rich Central European forest area. International Journal of Applied Earth Observation and Geoinformation, 52, 464-474 
Richter, R., \& Schläpfer, D. (2002). Geo-atmospheric processing of airborne imaging spectrometry data. Part 2: atmospheric/topographic correction. International Journal of Remote Sensing, 23, 2631-2649

Rodriguez-Galiano, V.F., Chica-Olmo, M., Abarca-Hernandez, F., Atkinson, P.M., \& Jeganathan, C. (2012). Random Forest classification of Mediterranean land cover using multi-seasonal imagery and multi-seasonal texture. Remote Sensing of Environment, 121, 93-107

Rouse Jr, J.W., Haas, R., Schell, J., \& Deering, D. (1974). Monitoring vegetation systems in the Great Plains with ERTS

Salisbury, J.W. (1986). Preliminary measurements of leaf spectral reflectance in the 8-14 $\mu \mathrm{m}$ region. International Journal of Remote Sensing, 7, 1879-1886

Sánchez-Azofeifa, G.A., Castro, K., Wright, S.J., Gamon, J., Kalacska, M., Rivard, B., Schnitzer, S.A., \& Feng, J.L. (2009). Differences in leaf traits, leaf internal structure, and spectral reflectance between two communities of lianas and trees: Implications for remote sensing in tropical environments. Remote Sensing of Environment, 113, 2076-2088

Sarrazin, M., van Aardt, J., Asner, G., McGlinchy, J., Messinger, D., \& Wu, J. (2012). Fusing small-footprint waveform LiDAR and hyperspectral data for canopy-level species classification and herbaceous biomass modeling in savanna ecosystems. Canadian Journal of Remote Sensing, 37, 653-665

Sayn-Wittgenstein, L. (1978). Recognition of tree species on aerial photographs

Schläpfer, D., \& Richter, R. (2011). Spectral polishing of high resolution imaging spectroscopy data. In, Proceedings of the 7th SIG-IS Workshop on Imaging Spectroscopy, Edinburgh, UK (pp. 11-13)

Schlerf, M., \& Atzberger, C. (2006). Inversion of a forest reflectance model to estimate structural canopy variables from hyperspectral remote sensing data. Remote Sensing of Environment, 100, 281-294

Schlerf, M., \& Atzberger, C. (2012). Vegetation Structure Retrieval in Beech and Spruce Forests Using Spectrodirectional Satellite Data. IEEE Journal of Selected Topics in Applied Earth Observations and Remote Sensing, 5, 8-17

Schlerf, M., Atzberger, C., Hill, J., Buddenbaum, H., Werner, W., \& Schüler, G. (2010). Retrieval of chlorophyll and nitrogen in Norway spruce (Picea abies L. Karst.) using imaging spectroscopy. International Journal of Applied Earth Observation and Geoinformation, 12, 17-26

Schölkopf, B., Burges, C.J., \& Smola, A.J. (1999). Advances in kernel methods: support vector learning. MIT press

Schwind, P., Schneider, M., \& Müller, R. (2014). Improving HySpex Sensor CoRegistration Accuracy using BRISK and Sensor-model based RANSAC. The 
International Archives of Photogrammetry, Remote Sensing and Spatial Information Sciences, 40, 371

Sehgal, V.K., Chakraborty, D., \& Sahoo, R.N. (2016). Inversion of radiative transfer model for retrieval of wheat biophysical parameters from broadband reflectance measurements. Information Processing in Agriculture, 3, 107-118 Senn, J., \& Suter, W. (2003). Ungulate browsing on silver fir (Abies alba) in the Swiss Alps: beliefs in search of supporting data. Forest ecology and management, 181, 151-164

Sethna, J. (2006). Statistical mechanics: entropy, order parameters, and complexity. Oxford University Press

Shang, X., \& Chazette, P. (2014). Interest of a full-waveform flown UV lidar to derive forest vertical structures and aboveground carbon. Forests, 5, 14541480

Shi, Y., Skidmore, A.K., Wang, T., Holzwarth, S., Heiden, U., Pinnel, N., Zhu, X., \& Heurich, M. (2018a). Tree species classification using plant functional traits from LiDAR and hyperspectral data. International Journal of Applied Earth Observation and Geoinformation, 73, 207-219

Shi, Y., Wang, T., Skidmore, A.K., \& Heurich, M. (2018b). Important LiDAR metrics for discriminating forest tree species in Central Europe. ISPRS Journal of Photogrammetry and Remote Sensing, 137, 163-174

Si, Y., Schlerf, M., Zurita-Milla, R., Skidmore, A., \& Wang, T. (2012). Mapping spatio-temporal variation of grassland quantity and quality using MERIS data and the PROSAIL model. Remote Sensing of Environment, 121, 415-425

Silba, J. (1986). Encyclopaedia coniferae. Phytologia memoirs. Vol. VIII. Moldenke and Moldenke, Corvallis, Oreg

Silveyra Gonzalez, R., Latifi, H., Weinacker, H., Dees, M., Koch, B., \& Heurich, M. (2018). Integrating LiDAR and high-resolution imagery for object-based mapping of forest habitats in a heterogeneous temperate forest landscape. International Journal of Remote Sensing, 1-26

Sims, D.A., \& Gamon, J.A. (2002). Relationships between leaf pigment content and spectral reflectance across a wide range of species, leaf structures and developmental stages. Remote Sensing of Environment, 81, 337-354

Singh, M., Evans, D., Tan, B.S., \& Nin, C.S. (2015). Mapping and characterizing selected canopy tree species at the Angkor World Heritage site in Cambodia using aerial data. PLoS One, 10, e0121558

Skidmore, A.K., Pettorelli, N., Coops, N.C., Geller, G.N., Hansen, M., Lucas, R., Mücher, C.A., O'Connor, B., Paganini, M., \& Pereira, H.M. (2015). 
Environmental science: agree on biodiversity metrics to track from space. Nature, 523, 403-405

Skowronek, S., Asner, G.P., \& Feilhauer, H. (2017). Performance of one-class classifiers for invasive species mapping using airborne imaging spectroscopy. Ecological Informatics, 37, 66-76

Smits, I., Prieditis, G., Dagis, S., \& Dubrovskis, D. (2012). Individual tree identification using different LIDAR and optical imagery data processing methods. Biosystems Inform Tech, 1, 19-24

Somers, B., \& Asner, G.P. (2013a). Invasive Species Mapping in Hawaiian Rainforests Using Multi-Temporal Hyperion Spaceborne Imaging Spectroscopy. IEEE Journal of Selected Topics in Applied Earth Observations and Remote Sensing, 6, 351-359

Somers, B., \& Asner, G.P. (2013b). Multi-temporal hyperspectral mixture analysis and feature selection for invasive species mapping in rainforests. Remote Sensing of Environment, 136, 14-27

Somers, B., \& Asner, G.P. (2014). Tree species mapping in tropical forests using multi-temporal imaging spectroscopy: Wavelength adaptive spectral mixture analysis. International Journal of Applied Earth Observation and Geoinformation, 31, 57-66

Sommer, C., Holzwarth, S., Heiden, U., Heurich, M., Müller, J., \& Mauser, W. (2016). Feature-based tree species classification using hyperspectral and Lidar data in the Bavarian Forest National Park. EARSeL eProceedings, 14, 49-70

Sothe, C., Dalponte, M., de Almeida, C.M., Schimalski, M.B., Lima, C.L., Liesenberg, V., Miyoshi, G.T., \& Tommaselli, A.M.G. (2019). Tree Species Classification in a Highly Diverse Subtropical Forest Integrating UAV-Based Photogrammetric Point Cloud and Hyperspectral Data. Remote Sensing, 11, 1338

Spurr, S.H. (1960). Photogrammetry and Photo-Interpretation. Soil Science, 90, 380

Stenzel, S., Fassnacht, F.E., Mack, B., \& Schmidtlein, S. (2017). Identification of high nature value grassland with remote sensing and minimal field data. Ecological Indicators, 74, 28-38

Sumnall, M.J., Hill, R.A., \& Hinsley, S.A. (2016). Comparison of small-footprint discrete return and full waveform airborne lidar data for estimating multiple forest variables. Remote Sensing of Environment, 173, 214-223

Suratman, M.N. (2012). Tree species diversity and forest stand structure of Pahang National Park, Malaysia. INTECH Open Access Publisher 
Suratno, A., Seielstad, C., \& Queen, L. (2009). Tree species identification in mixed coniferous forest using airborne laser scanning. ISPRS Journal of Photogrammetry and Remote Sensing, 64, 683-693

Swatantran, A., Dubayah, R., Roberts, D., Hofton, M., \& Blair, J.B. (2011). Mapping biomass and stress in the Sierra Nevada using lidar and hyperspectral data fusion. Remote Sensing of Environment, 115, 2917-2930

Teicher, M.H., Polcari, A., Fourligas, N., Vitaliano, G., \& Navalta, C.P. (2012). Hyperactivity persists in male and female adults with ADHD and remains a highly discriminative feature of the disorder: a case-control study. $B M C$ Psychiatry, 12, 190

Tian, J., Schneider, T., Straub, C., Kugler, F., \& Reinartz, P. (2017). Exploring Digital Surface Models from Nine Different Sensors for Forest Monitoring and Change Detection. Remote Sensing, 9, 287

Tinner, W., Colombaroli, D., Heiri, O., Henne, P.D., Steinacher, M., Untenecker, J., Vescovi, E., Allen, J.R., Carraro, G., \& Conedera, M. (2013). The past ecology of Abies alba provides new perspectives on future responses of silver fir forests to global warming. Ecological Monographs, 83, 419-439

Townsend, P.A., Foster, J.R., Chastain, R.A., \& Currie, W.S. (2003). Application of imaging spectroscopy to mapping canopy nitrogen in the forests of the central Appalachian Mountains using Hyperion and AVIRIS. Geoscience and Remote Sensing, IEEE Transactions on, 41, 1347-1354

Tuominen, S., Näsi, R., Honkavaara, E., Balazs, A., Hakala, T., Viljanen, N., Pölönen, I., Saari, H., \& Ojanen, H. (2018). Assessment of classifiers and remote sensing features of hyperspectral imagery and stereo-photogrammetric point clouds for recognition of tree species in a forest area of high species diversity. Remote Sensing, 10, 714

Ustin, S.L., Gitelson, A.A., Jacquemoud, S., Schaepman, M., Asner, G.P., Gamon, J.A., \& Zarco-Tejada, P. (2009). Retrieval of foliar information about plant pigment systems from high resolution spectroscopy. Remote Sensing of Environment, 113, Supplement 1, S67-S77

Vaglio Laurin, G., Cheung-Wai Chan, J., Chen, Q., Lindsell, J.A., Coomes, D.A., Guerriero, L., Del Frate, F., Miglietta, F., \& Valentini, R. (2014). Biodiversity mapping in a tropical West African forest with airborne hyperspectral data. PLoS One, 9, e97910

Vain, A., \& Kaasalainen, S. (2011). Correcting airborne laser scanning intensity data. Laser Scanning, Theory and Applications: InTech 
Vallet, P., \& Pérot, T. (2011). Silver fir stand productivity is enhanced when mixed with Norway spruce: evidence based on large-scale inventory data and a generic modelling approach. Journal of Vegetation Science, 22, 932-942

Vauhkonen, J., Korpela, I., Maltamo, M., \& Tokola, T. (2010a). Imputation of single-tree attributes using airborne laser scanning-based height, intensity, and alpha shape metrics. Remote Sensing of Environment, 114, 1263-1276

Vauhkonen, J., Ørka, H.O., Holmgren, J., Dalponte, M., Heinzel, J., \& Koch, B. (2014). Tree species recognition based on airborne laser scanning and complementary data sources. Forestry applications of airborne laser scanning (pp. 135-156): Springer

Vauhkonen, J., Tokola, T., Maltamo, M., \& Packalén, P. (2010b). Applied 3D texture features in ALS-based forest inventory. European Journal of Forest Research, 129, 803-811

Verhoef, W., \& Bach, H. (2007). Coupled soil-leaf-canopy and atmosphere radiative transfer modeling to simulate hyperspectral multi-angular surface reflectance and TOA radiance data. Remote Sensing of Environment, 109, 166-182

Vogelmann, J., Rock, B., \& Moss, D. (1993). Red edge spectral measurements from sugar maple leaves. TitleREMOTE SENSING, 14, 1563-1575

Vrška, T., Adam, D., Hort, L., Kolář, T., \& Janík, D. (2009). European beech (Fagus sylvatica L.) and silver fir (Abies alba Mill.) rotation in the Carpathians-A developmental cycle or a linear trend induced by man? Forest ecology and management, 258, 347-356

Wagner, F.H., Ferreira, M.P., Sanchez, A., Hirye, M.C.M., Zortea, M., Gloor, E., Phillips, O.L., de Souza, C.R., Shimabukuro, Y.E., \& Aragao, L. (2018). Individual tree crown delineation in a highly diverse tropical forest using very high resolution satellite images. ISPRS Journal of Photogrammetry and Remote Sensing, 145, 362-377

Wagner, W. (2010). Radiometric calibration of small-footprint full-waveform airborne laser scanner measurements: Basic physical concepts. ISPRS Journal of Photogrammetry and Remote Sensing, 65, 505-513

Wagner, W., Ullrich, A., Ducic, V., Melzer, T., \& Studnicka, N. (2006). Gaussian decomposition and calibration of a novel small-footprint full-waveform digitising airborne laser scanner. ISPRS Journal of Photogrammetry and Remote Sensing, 60, 100-112

Wang, Z., Skidmore, A.K., Darvishzadeh, R., \& Wang, T. (2018). Mapping forest canopy nitrogen content by inversion of coupled leaf-canopy radiative transfer 
models from airborne hyperspectral imagery. Agricultural and Forest Meteorology, 253, 247-260

Wang, Z., Skidmore, A.K., Wang, T., Darvishzadeh, R., \& Hearne, J. (2015). Applicability of the PROSPECT model for estimating protein and cellulose+ lignin in fresh leaves. Remote Sensing of Environment, 168, 205-218

Wang, Z., Wang, T., Darvishzadeh, R., Skidmore, A., Jones, S., Suarez, L., Woodgate, W., Heiden, U., Heurich, M., \& Hearne, J. (2016). Vegetation Indices for Mapping Canopy Foliar Nitrogen in a Mixed Temperate Forest. Remote Sensing, 8, 491

Waring, R.H. (1983). Estimating forest growth and efficiency in relation to canopy leaf area. Advances in ecological research (pp. 327-354): Elsevier

Waser, L., Ginzler, C., \& Rehush, N. (2017). Wall-to-Wall Tree Type Mapping from Countrywide Airborne Remote Sensing Surveys. Remote Sensing, 9, 766

Waser, L.T., Ginzler, C., Kuechler, M., Baltsavias, E., \& Hurni, L. (2011). Semiautomatic classification of tree species in different forest ecosystems by spectral and geometric variables derived from Airborne Digital Sensor (ADS40) and RC30 data. Remote Sensing of Environment, 115, 76-85

Waser, L.T., Klonus, S., Ehlers, M., Küchler, M., \& Jung, A. (2010). Potential of digital sensors for land cover and tree species classifications-a case study in the framework of the DGPF-project. Photogrammetrie-FernerkundungGeoinformation, 2010, 141-156

Waske, B. (2017). In-depth comparisons of MaxEnt, biased SVM and one-class SVM for one-class classification of remote sensing data AU - Mack, Benjamin. Remote Sensing Letters, 8, 290-299

Wasser, L., Day, R., Chasmer, L., \& Taylor, A. (2013). Influence of Vegetation Structure on Lidar-derived Canopy Height and Fractional Cover in Forested Riparian Buffers During Leaf-Off and Leaf-On Conditions. PLoS One, 8

Wiens, J., Sutter, R., Anderson, M., Blanchard, J., Barnett, A., AguilarAmuchastegui, N., Avery, C., \& Laine, S. (2009). Selecting and conserving lands for biodiversity: The role of remote sensing. Remote Sensing of Environment, 113, 1370-1381

Wollersheim, M., Collins, M.J., \& Leckie, D. (2011). Estimating boreal forest species type with airborne polarimetric synthetic aperture radar. International Journal of Remote Sensing, 32, 2481-2505

Yao, W., Krzystek, P., \& Heurich, M. (2012). Tree species classification and estimation of stem volume and DBH based on single tree extraction by exploiting airborne full-waveform LiDAR data. Remote Sensing of Environment, 123, 368-380 
Yao, W., Krzystek, P., \& Heurich, M. (2013). Enhanced detection of 3D individual trees in forested areas using airborne full-waveform LiDAR data by combining normalized cuts with spatial density clustering. ISPRS Annals of Photogrammetry, Remote Sensing and Spatial Information Sciences, 1, 349-354

Yin, D., \& Wang, L. (2016). How to assess the accuracy of the individual treebased forest inventory derived from remotely sensed data: a review. International Journal of Remote Sensing, 37, 4521-4553

Zarco-Tejada, P.J., Miller, J.R., Mohammed, G.H., \& Noland, T.L. (2000). Chlorophyll fluorescence effects on vegetation apparent reflectance: I. Leaflevel measurements and model simulation. Remote Sensing of Environment, $74,582-595$

Zhang, C., Selch, D., \& Cooper, H. (2015). A Framework to Combine Three Remotely Sensed Data Sources for Vegetation Mapping in the Central Florida Everglades. Wetlands, 1-13

Zhang, K., \& Hu, B. (2012). Individual urban tree species classification using very high spatial resolution airborne multi-spectral imagery using longitudinal profiles. Remote Sensing, 4, 1741-1757

Zhang, Z., Kazakova, A., Moskal, L., \& Styers, D. (2016). Object-Based Tree Species Classification in Urban Ecosystems Using LiDAR and Hyperspectral Data. Forests, 7, 122

Zhao, K.G., Valle, D., Popescu, S., Zhang, X.S., \& Mallick, B. (2013). Hyperspectral remote sensing of plant biochemistry using Bayesian model averaging with variable and band selection. Remote Sensing of Environment, $132,102-119$ 


\section{Summary}

The accurate identification of tree species is critical for the management of forest ecosystems. Mapping of tree species is an important task as it can assist a wide range of environmental applications, such as biodiversity monitoring, ecosystem services assessment, invasive species detection, and sustainable forest management.

Compared to the conventional approaches based on labor-intensive field measurements, remote sensing has supplied a large variety of cutting-edge techniques to accomplish forest inventory. However, individual tree species classification in natural mixed forests, as it is typical in central Europe, is still a challenging task. High spectral and structural intra-species variability and interspecies similarity, due to phenological effects, differences in tree age and openness of canopies, shadowing effects, and environment variability, restrict tree species separability. An in-depth understanding of the relationship between species-specific features and remote sensing observations for tree species classification needs further investigation.

This thesis aimed to accurately map the species of individual trees using multisource remotely sensed data, including aerial photographs, airborne LiDAR and hyperspectral data. The research in the thesis firstly evaluated the performance of geometric and radiometric metrics from airborne LiDAR data under leaf-on and leaf-off conditions for individual tree species discrimination. The results empathized the importance of intensity-related LiDAR metrics for tree species identification under both leaf-on and leaf-off conditions. Then, the thesis examined whether multi-temporal digital CIR orthophotos could be used to further increase the accuracy of airborne LiDAR-based individual tree species mapping. The results showed that the texture features generated from multitemporal digital CIR orthophotos under different view-illumination conditions are species-specific. Combining these texture features with LiDAR metrics significantly improved the accuracy of individual tree species mapping. To explore more valuable species-specific features, the thesis consequently integrated three plant functional traits (i.e. equivalent water thickness, leaf mass per area and leaf chlorophyll) retrieved from hyperspectral data with hyperspectral derived spectral features and airborne LiDAR derived metrics for mapping five tree species. Three selected plant functional traits were accurately retrieved using radiative transfer model and further improved the accuracy of tree species classification. Eventually, the thesis focused on an important tree species 
- silver fir, and accurately mapped individuals of this species based on one-class classifiers using integrated airborne hyperspectral and LiDAR data. The mapping results provided the references locating the areas with a high occurrence probability of silver fir trees and hence increase the efficiency in subsequent field campaigns for forest management and biodiversity monitoring.

This thesis explored the potential of various remotely sensed datasets for individual tree species mapping. The methodologies and findings in this thesis can be applied in the mapping of other tree species, which enriches the knowledge of species-specific characteristics and related remotely sensed signatures. The emerging of UAVs and the upcoming hyperspectral missions such as EnMAP and HySPIRI deliver valuable datasets with multi-scale coverage and revisit observations, which can be used for mapping the diversity of tree species at stand or regional level. 


\section{Samenvatting}

De nauwkeurige identificatie van boomsoorten is van cruciaal belang voor het beheer van bosecosystemen. Het in kaart brengen van boomsoorten is een belangrijke taak omdat het een breed scala aan milieutoepassingen kan ondersteunen, zoals monitoring van de biodiversiteit, beoordeling van ecosysteemdiensten, detectie van invasieve soorten en duurzaam bosbeheer.

In vergelijking met de conventionele benaderingen op basis van arbeidsintensieve veldmetingen, heeft teledetectie een grote verscheidenheid aan geavanceerde technieken opgeleverd om bosinventarisatie uit te voeren. De individuele classificatie van boomsoorten in natuurlijk gemengde bossen, zoals gebruikelijk in centraal Europa, is echter nog steeds een uitdagende taak. Hoge spectrale en structurele intraspecifieke variabiliteit en gelijkenis tussen species, vanwege fenologische effecten, verschillen in structuur leeftijd en openheid van luifels, schaduweffecten en milieu variabiliteit beperken de mogelijkheid om boomsoorten te kunnen onderscheiden. Een diepgaand begrip van de relatie tussen soortspecifieke kenmerken en teledetectieobservaties voor classificatie van boomsoorten moeten nader worden onderzocht.

Dit proefschrift heeft als doel om de soorten individuele bomen nauwkeurig in kaart te brengen met behulp van op afstand gedetecteerde gegevens uit meerdere bronnen, waaronder luchtfoto's, LiDAR in de lucht en hyperspectrale gegevens. Het onderzoek in het proefschrift evalueerde eerst de prestaties van geometrische en radiometrische metrieken van LiDAR-gegevens in de lucht onder leaf-on en leaf-off omstandigheden voor individuele boomsoortendiscriminatie. De resultaten benadrukten het belang van intensiteitsgerelateerde LiDARstatistieken voor de identificatie van boomsoorten onder zowel leaf-on als leafoff omstandigheden. Vervolgens is in het proefschrift onderzocht of multitemporele digitale CIR-orthofoto's kunnen worden gebruikt om de nauwkeurigheid van op LiDAR gebaseerde individuele boomsoortenkaarten verder te vergroten. De resultaten toonden aan dat de textuurkenmerken gegenereerd door multi-temporele digitale CIR-orthofoto's onder verschillende belichtingsomstandigheden soortspecifiek zijn. De combinatie van deze structuur functies met LiDAR metrics een aanzienlijke verbetering van $d$ de nauwkeurigheid van individual boomsoorten mapping. Om meer waardevolle soortspecifieke kenmerken te onderzoeken, integreerde het proefschrift daarom drie plantfunctionele eigenschappen (dwz equivalente waterdikte, bladmassa per oppervlakte en bladchlorofyl) verkregen uit hyperspectrale gegevens met 
hyperspectrale afgeleide spectrale kenmerken en uit de lucht afkomstige LiDARmetrieken voor het in kaart brengen van vijf boomsoorten. Drie geselecteerde functionele kenmerken van planten werden nauwkeurig opgehaald met behulp van het stralingsoverdrachtmodel en verbeterden verder de nauwkeurigheid van de boomsoortclassificatie. Uiteindelijk richt het proefschrift zich op een belangrijke soort - zilver spar, en nauwkeurig in kaart gebracht van deze soort op basis van één-class classifiers met behulp van geïntegreerde airborne hyperspectrale en LiDAR data. De karteringsresultaten verschaffen verwijzingen om de gebieden met een grote optredingswaarschijnlijkheid voor de zilver sparren te lokaliseren en daarmee de efficiëntie volgende gebied campagnes bosbouw en daarmee de monitoring van biodiversiteits te verhogen.

Dit proefschrift onderzocht het potentieel van verschillende op afstand gedetecteerde datasets voor het in kaart brengen van individuele boomsoorten. De methodologieën en bevindingen in dit proefschrift kunnen worden toegepast bij het in kaart brengen van andere boomsoorten, wat de kennis van soortspecifieke kenmerken en gerelateerde op afstand gedetecteerde handtekeningen verrijkt. De opkomst van UAV's en de aankomende hyperspectrale missies zoals EnMAP en HySPIRI leveren waardevolle datasets met multi-schaal dekking en herwaarderingswaarnemingen, die kunnen worden gebruikt voor het in kaart brengen van de diversiteit van boomsoorten op stand of regionaal niveau. 


\section{Biography}

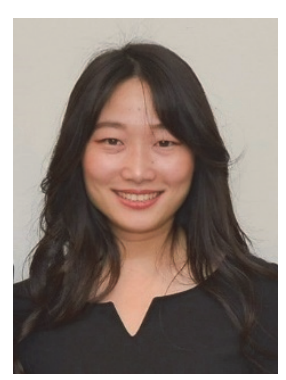

Yifang Shi was born on 21 July, 1990 in Henan, China. In June 2012, she received her MSc degree in Cartography and Geographic Information Engineering from Fuzhou University, China. In the same year, she was awarded a four-year doctorial scholarship from the China Scholarship Council (CSC), and started to pursue her $\mathrm{PhD}$ at the Faculty of GeoInformation Science and Earth Observation (ITC), University of Twente, the Netherlands. From October 2017 till March 2018, she was a scientific visitor at German Aerospace Center (DLR), Germany. Her research interests lie in multi-source remotely sensed data for forestry applications, feature extraction and tree species mapping using hyperspectral and LiDAR data. 